Illinois State University

ISU ReD: Research and eData

Theses and Dissertations

3-22-2019

\title{
Incidental Cardiac Arrhythmia Identification With Consumer Grade Heart Rate Monitors: A Case Study
}

Neal C. Phifer

Illinois State University, phifer_family@icloud.com

Follow this and additional works at: https://ir.library.illinoisstate.edu/etd

Part of the Physiology Commons

\section{Recommended Citation}

Phifer, Neal C., "Incidental Cardiac Arrhythmia Identification With Consumer Grade Heart Rate Monitors: A Case Study" (2019). Theses and Dissertations. 1068.

https://ir.library.illinoisstate.edu/etd/1068

This Thesis is brought to you for free and open access by ISU ReD: Research and eData. It has been accepted for inclusion in Theses and Dissertations by an authorized administrator of ISU ReD: Research and eData. For more information, please contact ISUReD@ilstu.edu. 


\title{
INCIDENTAL CARDIAC ARRHYTHMIA IDENTIFICATION WITH CONSUMER GRADE HEART RATE MONITORS: A CASE STUDY
}

\author{
NEAL C. PHIFER
}

\section{Pages}

PURPOSE: The purpose of the study was to examine instances of using consumer grade chest strap type heart rate monitor (HRM) data as a means of identifying otherwise asymptomatic individuals with possible cardiac arrhythmias as examined across four division one collegiate sports teams.

METHODS: Heart rate activity was tracked across an entire season of four different division one collegiate teams (men's basketball, women's basketball, women's soccer and women's volleyball). A total of 65 athletes were equipped with heart rate monitors (Polar Team Pro Sensor; Polar Electro Inc., Bethpage, NY USA) that were worn as allowed during practices and games. Data were collected using chest strap mounted activity sensors (Polar Team Pro sensors attached to Polar Team Pro soft strap). Monitors were maintained by coaching staff and provided to athletes at the beginning of every session and recovered at the conclusion for data upload and analysis. Monitors measured heart rate, accelerations, speed, and distance. All data were uploaded to the monitor's manufacturer website and then collected by the researchers for analysis.

RESULTS: During retrospective analysis of recorded data it was identified that an athlete evidenced a heart rate response that could have possibly indicated the presence of some type of arrhythmia. The athlete identified during analysis never made evident any symptoms that would 
indicate the presence of an abnormality. Given the frequency and number of occurrences, athlete was referred for cardiologist and electrophysiologist evaluation and was ultimately diagnosed with exercise induced supraventricular tachycardia (SVT).

CONCLUSIONS: Although not meant as a means to diagnose a specific cardiac condition it appears that identification of individuals, which would otherwise be asymptomatic, with some sort of arrhythmia is plausible through the use of analysis of HRM data.

KEYWORDS: arrhythmia, asymptomatic, occurrence, monitor, artifact 
INCIDENTAL CARDIAC ARRHYTHMIA IDENTIFICATION WITH CONSUMER GRADE

HEART RATE MONITORS: A CASE STUDY

NEAL C. PHIFER

\begin{abstract}
A Thesis Submitted in Partial
Fulfillment of the Requirements

for the Degree of

MASTER OF SCIENCE
\end{abstract}

School of Kinesiology and Recreation

ILLINOIS STATE UNIVERSITY

2019 
Copyright 2019 Neal C. Phifer 
INCIDENTAL CARDIAC ARRHYTHMIA IDENTIFICATION WITH CONSUMER GRADE

HEART RATE MONITORS: A CASE STUDY

NEAL C. PHIFER

COMMITTEE MEMBERS:

Dale D. Brown, Chair

Kelly R. Laurson

Skip M. Williams

Emily Jones

Karen K. Dennis 


\section{ACKNOWLEDGMENTS}

I would like to take this opportunity to provide thanks for those that had a part in making this thesis possible. To begin with I would like to thank my committee members as they provided much needed guidance and support throughout this process. To say there was patience would be an understatement. Their perspective proved invaluable.

In addition to my committee members I would like to make it known that this could not have happened without the support and dedication of the women's volleyball staff. Our many communications were absolutely vital to both the crafting of this thesis but also to maintain the health and well-being of their athletes. Specifically, I would like to commend and thank Megan Smith, Ryan Swenson, and Leah Johnson.

Lastly and certainly not least I would like to thank the subject of the case study itself.

N.C.P. 


\section{CONTENTS}

Page

ACKNOWLEDGEMENTS

$\begin{array}{lll}\text { TABLES } & \text { iii }\end{array}$

FIGURES iv

CHAPTER I: INCIDENTAL CARDIAC ARRHYTHMIA IDENTIFICATION WITH CONSUMER GRADE HEART RATE MONITORS: A CASE STUDY 1

Introduction 1

$\begin{array}{ll}\text { Methods } & 4\end{array}$

Presentation of Case $\quad 5$

$\begin{array}{ll}\text { Discussion } & 7\end{array}$

$\begin{array}{ll}\text { Retrospective Analysis } & 7\end{array}$

$\begin{array}{lr}\text { Course of Action } & 8\end{array}$

$\begin{array}{ll}\text { Additional Observations } & 11\end{array}$

$\begin{array}{ll}\text { Expanded Screening } & 22\end{array}$

$\begin{array}{ll}\text { Conclusions } & 27\end{array}$

CHAPTER II: REVIEW OF LITERATURE 29

$\begin{array}{ll}\text { REFERENCES } & 33\end{array}$

APPENDIX A: COMPOSITE/DESCRIPTIVE IMAGES 35

APPENDIX B: RECORDED SESSION IMAGES 39

APPENDIX C: NUMERIC ANALYSIS OF "ARRHYTHMIA" DATA 66

APPENDIX D: DESCRIPTIONS OF COULMNS FOUND IN TABLE 1 


\section{TABLES}

Table Page

1. Occurrence statistical values

2. Number of instances in excess of $205 \mathrm{bpm}$ 


\section{FIGURES}

Figure

Page

1. HR trace recorded during session on 1 Feb 2019, note 2 instances of sustained elevated HR

2. Four other athlete HR traces have been included to illustrate the deviation from other athlete HRs as they were performing similar exercises/drills

3. Simultaneous HR recording by 2 Polar Team Pro sensors

4. Elevated HR occurrence at approximately 18 minutes into the session

5. Close up of initiation and termination of occurrence viewed in HR tracing format

6. Showing a 10 second duration close-up of the termination of the SVT occurrence and the same image mirrored and inverted

7. Illustrating the same images from Figure 5, but the one on the right has been inverted and rotated 180 degrees

8. Showing a comparison between BPM (top) and RRI (bottom) and their inverse graphical relationship

9. Illustrates close up of initiation and termination of occurrence as viewed in RRI Format

10. Illustrating RRI details of an occurrence taking place below the maximum calculated HR for the subject

11. Illustrating elevated prolonged HR and subsequent RRI analysis

12. Numeric analysis of arrhythmia data taken from HR and RRI information (does not show all occurrences, complete table showing all occurrences is found in Appendix C)

13. Showing copy of flow chart from article by Gajda, et al. (Gajda et al., 2018a), that displays a recommended sequence with arrhythmia is detected using HRM 


\section{CHAPTER I: INCIDENTAL CARDIAC ARRHYTHMIA IDENTIFICATION WITH CONSUMER GRADE HEART RATE MONITORS: A CASE STUDY}

\section{Introduction}

There is a constant need for data around the health and fitness industry. This data can span from simple monitoring of one's heart rate (HR) to in depth analysis of variables that can include but are not limited to accelerations, speed, altitude, etc. The overall outcome of this analysis can assist an individual to be become more physically fit, lose weight or any other myriad of fitness improvements, or under more specific analysis, to try to either improve an athlete's performance or to improve the performance of an entire team. There are an abundance types of variables to be analyzed, but one of the more often used is simple heart rate, usually measured in either percent of maximum HR or beats per minute (BPM). With the advancement of HR monitoring technologies, devices are now available that provide near instantaneous information about changes in heart rate based on response to increased or decreased workload. With this in mind comes the importance of interpreting the data that is presented, not necessarily in the sole aspect of improving health, but with the possibility of identifying other conditions.

Cardiac arrhythmias, more specifically in regards to this case study, a classification of arrhythmia known as supraventricular tachycardia (SVT), is broadly defined as an abnormally fast heartbeat that originates above the ventricles, in the atria or atrioventricular (AV) node. A resting heart rate in excess of $100 \mathrm{bpm}$ is considered to be tachycardia (tak-ih-KAHR-dee-uh). SVT is an arrhythmia that includes several forms of heart rhythm problems (heart arrhythmias) that originate above the ventricles (supraventricular) in the atria or AV node. This can occur when the electrical impulses that coordinate your heartbeat are not properly conducted or routed 
through the heart ("Supraventricular tachycardia - Symptoms and causes," n.d.). Another definition would be, "Supraventricular tachycardia (SVT) refers to rapid rhythms that originate and are sustained in atrial or atrioventricular nodal tissue, and then transmit through the bundle of His and cause rapid ventricular response"(Helton, 2015).

Of the studies performed concerning the applicability of HRMs for identifying arrhythmias, it would appear that most of them use subjects that are symptomatic, as indicated by either the athlete's/participant's own HRM outputs or physically manifested symptoms. The subjects are then tested against both a HRM and either an electrocardiogram (ECG) and/or Holter monitor.

An example of this would be a study published in 2018 by Gajda, et al. Their study used endurance athletes as subjects all of which had been referred to the research institute for study. The athletes were fitted with both a Holter monitor and their own HRM that they had used to record their suspected event. Once equipped the subjects performed a workout similar to the one that caused the suspected arrhythmia. Their overall findings were that the majority of the subjects $(99.3 \%)$ that were referred had no correlation between their HR readings and the Holter monitor output. For clarification, some of the subjects only showed significant readings on the Holter monitor while others only showed significant readings from their respective HR monitors. Only 1 of the subjects tested evidenced an arrhythmia that was identified and correlated between both the HRM and Holter monitor. (Gajda, Biernacka, \& Drygas, 2018)

Another study which would support the use of HRMs as supporting evidence of possible arrhythmia would be a 2017 article by Thabouillot et al. That case study outlines a single individual who presented with symptoms (decreased running performance, and eventually losing consciousness) after completing a $10 \mathrm{~km}$ run/race. Upon screening of data from the HRM it was 
discovered that a tachycardia episode had occurred during the race. Further testing was completed to include a stress test while the subject wore both his HRM and a Holter monitor. During the testing, atrial fibrillation was recorded on both the HRM and the Holter monitor. In summary their case study states that non-medical devices such as connected watches, can be helpful to diagnose arrhythmias (Thabouillot et al., 2017). In this instance though atrial fibrillation was identified and not SVT, as is the case in this particular study.

A study by Maurer et al., identified that the percentages of SVT were quite similar when viewed between genders under the age of 50 with males at $6.0 \%$ and females at $6.3 \%$ of the test population having recorded occurrences of SVT. Also of note was that there was an increase in the prevalence of SVT in men across the age groups identified but remained consistent across the different age groups for women. Interestingly enough the population with the smallest incidence of SVT would be females who were under the age of 50 and was actually non-existent in their study for anyone under the age of 20. As for complications for resulting from SVT, according to the Mayo Clinic, if it is left untreated and depending on duration and frequency of episode can lead to a weakening of some of the structures within the heart which could ultimately lead to heart failure("Supraventricular tachycardia - Symptoms and causes," n.d.)

This case study presents a unique opportunity through the use of HRMs for monitoring performance of collegiate level athletes through data analytics. More specifically it allowed for the almost inadvertent identification of an athlete with a possible arrhythmia. The purpose of this study was to examine an instance of using data from chest strap type HRM as a means of identifying an otherwise asymptomatic individual with an arrhythmia. 


\section{Methods}

Four division one sports teams at a mid-western university were monitored using the Polar Team Pro system for analysis of HR and performance characteristics. Sixty-five athletes were monitored during practices and games (when allowed), totaling 891 recorded sessions, with data analytics being performed on a routine basis to identify trends in training load and performance. Once analyzed the data would be formatted and presented to respective coaching staffs for possible use in coaching decisions.

Archived participant data from the Polar Team Pro Heart Rate Monitoring System was used as the primary data source for this research/case study. The Polar Team2 Pro Heart Rate Monitoring System consists of the following equipment:

iPad - The iPad was used for recording sessions (practices or games as allowed), and provided real-time visibility of athlete performance/comparison.

Base Station/Transmitter Charging Station - The base station acts as a wireless router and provides communication between the transmitter charging station and the computer software allowing for the upload of heart rate data stored on the heart rate transmitters. The heart rate transmitters were placed in the charging station after every use, which would sync with the base station to allow data to be uploaded to the computer software.

Polar Team Pro Soft Strap/Polar Fitted Shirt - A fabric, electrode strap/shirt for the Base Station to receive necessary information on heart activity. It is placed around the chest or worn as a shirt with the transmitter intact to the strap/shirt.

Polar Team Pro Sensor - Sensing device that was affixed to the Polar Team Pro Soft Strap or Fitted Shirt. The device was the actual data recording point, it contains the sensors to determine the HR, acceleration, GPS position. Each sensor was assigned to an athlete to ensure that data collected was representative of the athlete assigned.

The following variables were recorded and evaluated using the software:

Heart rate

Length of time at each intensity zone Individual maximal heart rate

Total distance

Maximum speed
Intensity (i.e. heart rate training zones)

Total calorie expenditure

GPS Position

Distance per minute

Average speed 
Number of sprints

Number of accelerations (zones 1-5)

Recovery load
Distance in speed zones (zones 1-5)

Number of decelerations (zones 1-5)

Total training load

The information was displayed on a designated iPad for only the investigator collecting data to see.

The equipment was used by the athlete's sport coach/training staff during training/practice/game situations. The data was then transferred from the transmitters and base station and stored within the software system and subsequently analyzed. Data was exported into a Microsoft Excel spreadsheet from the Polar website, sorted by team, and formatted to provide performance information to the respective coaching staffs.

\section{Presentation of Case}

On 1 Feb 2019 while observing a practice for women's volleyball it was noticed that a 19 year old athlete had an unusually high HR (peaking at $123 \%$ or 233 BPM). This was identified on the iPad that was used to track performance data on all of the athletes in attendance in real time. Once the athlete was identified, she was observed without interaction for signs of distress, excessive heavy breathing, pallor changes, or any sort of balance issues that would indicate dizziness or syncope. After a short time HR returned to a normal level that would be commensurate with the exercises/drills being performed. Attention was paid to HR levels for the remainder of practice, not just on the athlete in question but on all of the athletes participating, with no noticeable indications. The amount of time that the athlete spent with an elevated HR was long enough to be considered outside of a normal short duration spike in HR due to excessive short-term cardiovascular effort or connectivity (artifact). The possibility of equipment error was also considered.

Once practice had been completed and the data uploaded to the system, it was analyzed to view the anomaly in greater detail. Two distinct occurrences can be observed in Figure 1 at about 
the 00:31:36 mark and one at around the 01:01:36 mark. The first occurrence lasted approximately 1 minute, and the second lasted for much longer (approximately 5 minutes) and at a higher HR.

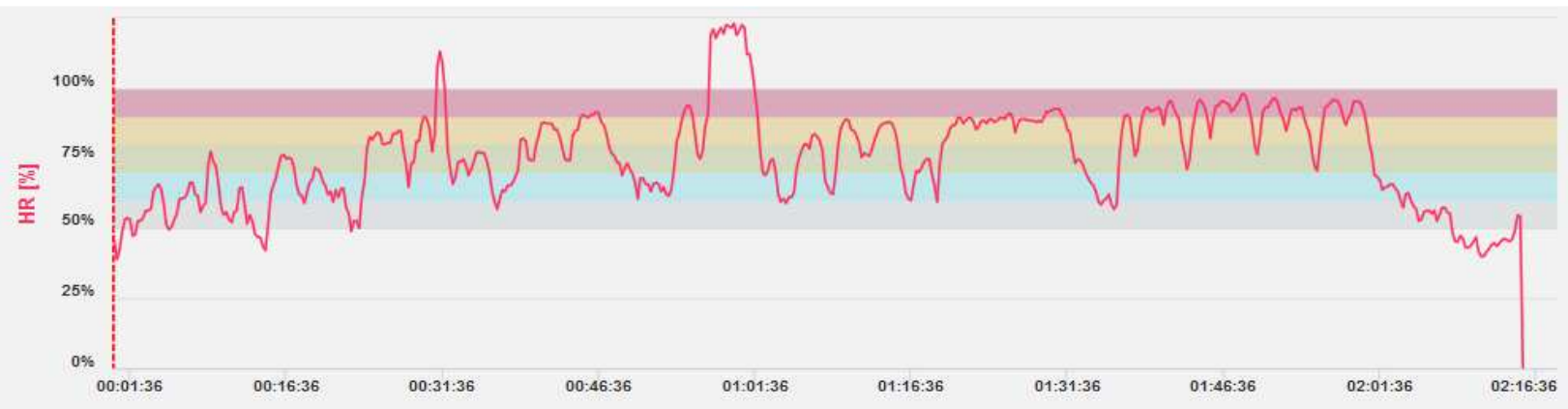

Figure 1: HR trace recorded during session on $1 \mathrm{Feb} 2019$, note 2 instances of sustained elevated HR

The athlete's HR trace was compared with four other athletes who were attending practice at the same time. Figure 2 shows that there is a marked difference in the subject's HR as compared to the other athletes at the time of the occurrences.

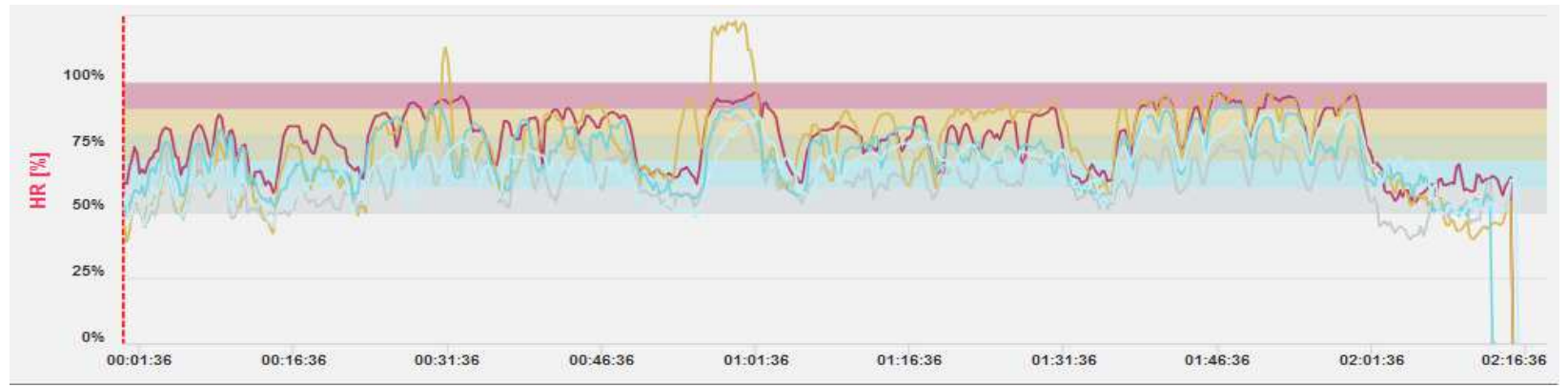

Figure 2: Four other athlete HR traces have been included to illustrate the deviation from other athlete HRs as they were performing similar exercises/drills 


\section{Discussion}

\section{Retrospective Analysis}

With further analysis of the data previously collected, it was revealed that the identified subject had taken part in 182 recorded activity sessions since the HRM system had been put into use. Of these 182 sessions a total of 73 sessions (40.1\%) were identified to have occurrences of sustained elevated HR, which could indicate some sort of arrhythmia. Five of these sessions were since discounted as the occurrences were determined to be artifact, which is defined later. The discounted sessions left a total of 68 sessions (37.3\%) that had data that would indicate some sort of possible arrhythmic episode had taken place.

These sessions were identified due to the maximum HR percentage exceeding $100 \%$ of $\mathrm{HR}$ as determined for this athlete. In this case the maximum HR was entered into the system as 190 beats per minute (bpm). The HR would have to remain above the 100\% threshold long enough for the value to be visible on the HR trace image, in this case in excess of 1 second. Another, slightly more subjective, criteria was that a comparison was made between the athlete in question and other athletes recorded during the same session performing similar exercises/drills. Visual observation of the HR trace that indicated a marked departure of HR from athletes performing at the same time were noted. R-R interval (RRI) data was also used to identify occurrences. RRI is a measurement of time between " $R$ " waves during consecutive cardiac cycles, also known as beat to beat interval.

Of the sessions, that the subject participated in, that were identified as meeting criteria, it was determined that multiple occurrences of sustained elevated HR had taken place in 33 of these sessions. The number of multiple occurrences ranged from two to five during a recorded session. Appendix B is a collection of all of the recorded sessions that contain identified elevated 
HR occurrences. There appears to be no prevalence or trend of when the occurrences occur during a session. Some were noted during a specific drill while others were noted to take place at the end of a drill. As almost all of the recorded sessions vary in time of recording or time of day recorded it would be difficult to draw a conclusion that either one of these variables would play a considerable part in determining likelihood of when an occurrence would take place.

Of note was the comparison of number of sessions that took place during a game setting in relation to the number of sessions during a practice setting. Twenty-four of the 57 recorded sessions, that met the visual criteria mentioned previously, were annotated as being a game or match. This accounts for $42.1 \%$, with 13 of these game sessions (54.2\%) having multiple occurrences. In comparison, 33 sessions, or the remaining $57.9 \%$, were identified as practice sessions, of which 10 had multiple occurrences this equates to $30.3 \%$. Gajda et al stated that this increase in frequency during game settings could be associated "... sometimes with participation in a competition, which increased the athletes' emotions as they maximally exerted themselves." (Gajda et al., 2018a). In a 2015 article, Helton stated that "The patient should be asked about precipitating factors, such as caffeine or other stimulant use, stress, and exercise. Onset with activity or a history of cardiac disease suggests a ventricular origin to the tachycardia rather than SVT, and these patients should be evaluated for underlying heart disease" (Helton, 2015). These two viewpoints contribute that there could be a psychological factor to the instigation of some of the occurrences happening more frequently during a competition setting.

\section{Course of Action}

The elevated heart rate data was forwarded to the data analytics program director who made contact with the coaching staff of the team to make them aware of the findings. The coaching and athletic training staff made the decision to contact the team physician for guidance. 
It was determined that the athlete in question would have an evaluation by a cardiologist for further screening and a possible course of action. After initial consultation with the team physician, the athlete was provided with clearance to continue with practice without restriction. During the subsequent practice sessions the real-time feed from the HRM was observed in a more continuous fashion.

During the first subsequent session the athlete was fitted with an additional HRM, resulting in 2 identical HRMs being worn simultaneously. This was done to determine if a possible equipment error was to be blamed for the anomaly. An additional identical sensor was given to the athlete and worn during practice. During this practice session another occurrence of sustained elevated heart rate was recorded. This recording is shown in the Figure 3 below. It can be seen that almost an identical tracing is recorded for the duration of the session to include the area of elevated HR. With this being the case it would appear that the HR monitor used was in good working order.

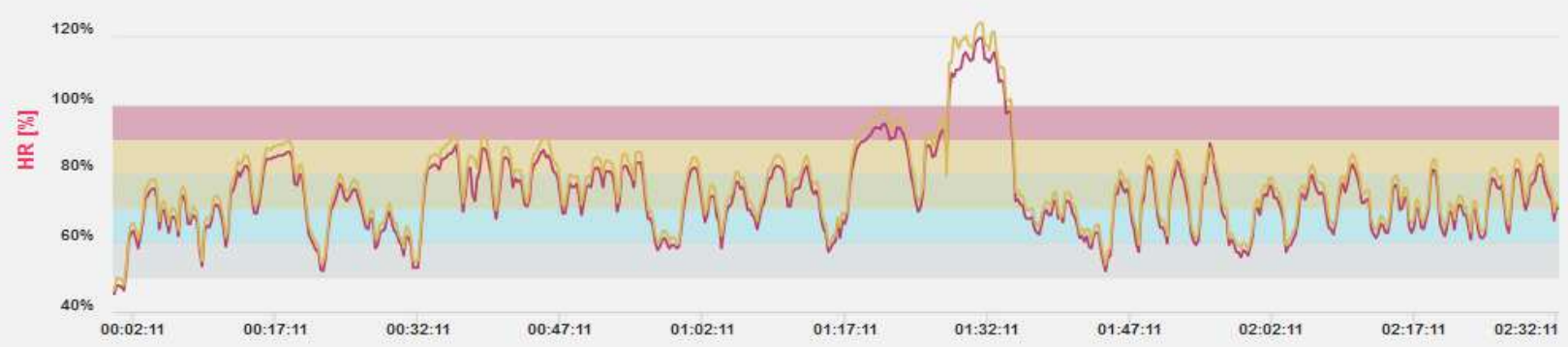

Figure 3: Simultaneous HR recording by 2 Polar Team Pro sensors

In addition to being provided with initial clearance to continue practicing, further testing was prescribed in the form of an ECG and the administration of a Holter monitor to record the athlete's cardiac function for 48 hours. Another occurrence of sustained elevated HR was recorded during the wearing of the HR monitor (Figure 4). By analyzing the recorded session a 
relatively precise time and duration of observations was sent to the cardiologist to assist with verifying that an arrhythmia occurrence had been observed.

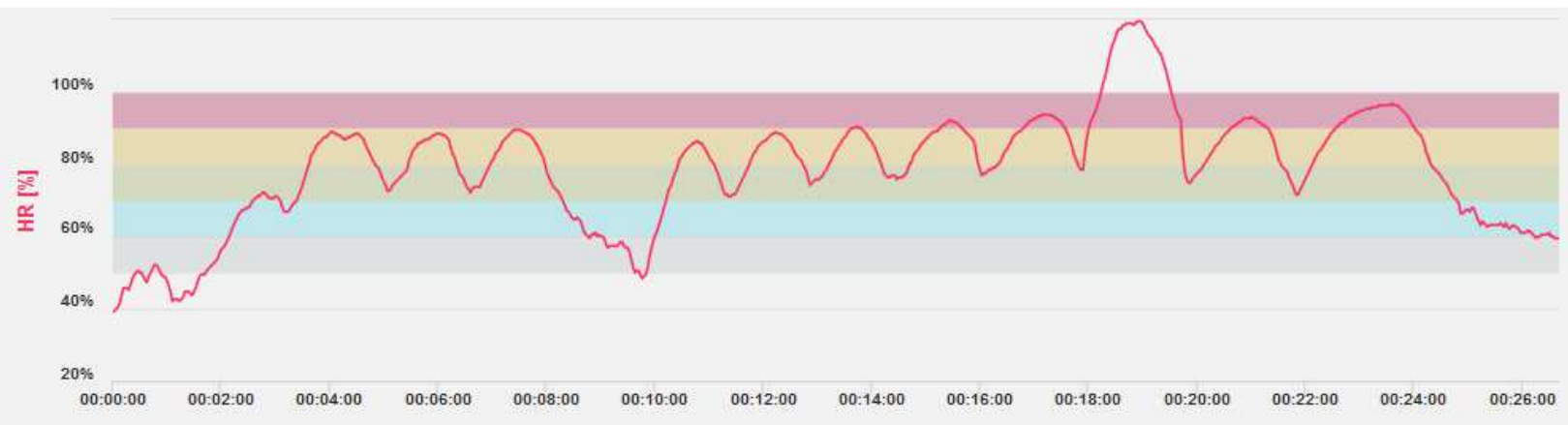

Figure 4: Elevated HR occurrence at approximately 18 minutes into the session

Initial results from Holter monitor data indicated the presence of an exercise induced SVT occurrence but was overall inconclusive as to the actual cause of the arrhythmia. With this information the subject was referred to an electrophysiologist for further clarification and analysis of the data that had been collected.

The electrophysiologist provided guidance in regards to procedures during practice or game when an occurrence takes place. It was recommended that constant monitoring of HR take place and that in the event of an occurrence the subject was to be removed from the session immediately until HR had returned to normal. During first occurrence since the new guidance was put into place, approximately 10 seconds lapsed from the time that the occurrence was noticed to when the athlete was removed from the session. The subject's HR declined from a peak of $221 \mathrm{bpm}$ down to $74 \mathrm{bpm}$ in just under 2 minutes. After returning to practice subject showed a "normal" HR profile much like before said episode occurred. Further analysis of more occurrences identified during monitoring would need to be accomplished to determine if removal from the practice session does in fact limit the duration of the length of the occurrence. 
Analysis indicated that the average $\mathrm{HR}$ at the time of occurrence initiation was approximately $162 \mathrm{bpm}(85 \%$ of $\max )$. During monitoring there were no indications that sustained HR above $90 \%$ is indicative of initiation of an occurrence. This information can be used to avoid focusing on times when the athlete's HR is already close to the maximum calculated HR as a precursor to an occurrence taking place. Currently there is no method of assessing rate of change, or slope, when viewing the real time data. That being said, close monitoring of rapid increases of HR become very important in trying to determine when an occurrence is beginning.

\section{Additional Observations}

During analysis of the recorded episodes from the subject, an observation was made when examining the initiation and termination of the SVT occurrences. Aside from determining slope and duration for both beginning and end of the episode, it was noticed that a sort of "telltale" marker was present on a majority of the recordings. There was a distinct curvature to both the initial increase of HR and a point where HR would make a marked return to levels commensurate with the exercise/drill being performed (Figure 5). 


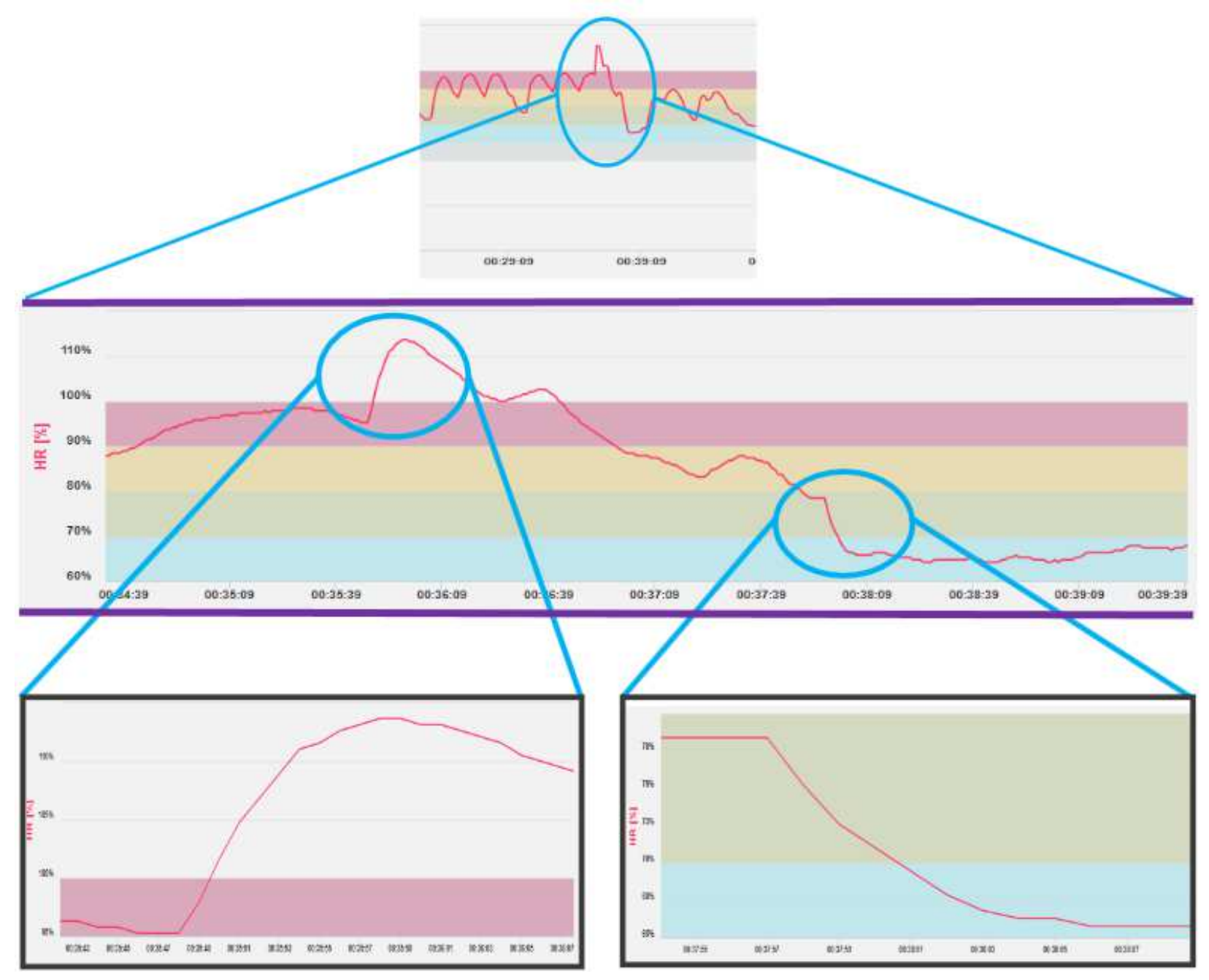

Figure 5: Close up of initiation and termination of occurrence viewed in HR tracing format

Once these curvatures had been identified further analysis was performed and by capturing an image, lasting approximately 10 seconds, and orienting the termination curve to match the orientation of the initiation curve it was noticed that the angles and curves were decidedly similar (Figures 6-7). 

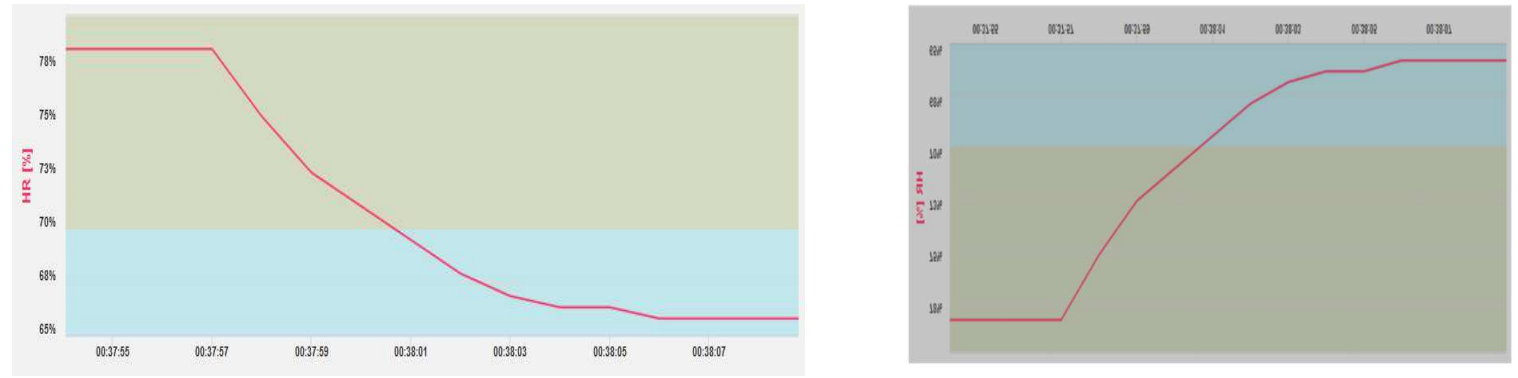

Figure 6: Showing a 10 second duration close-up of the termination of the SVT occurrence and the same image mirrored and inverted
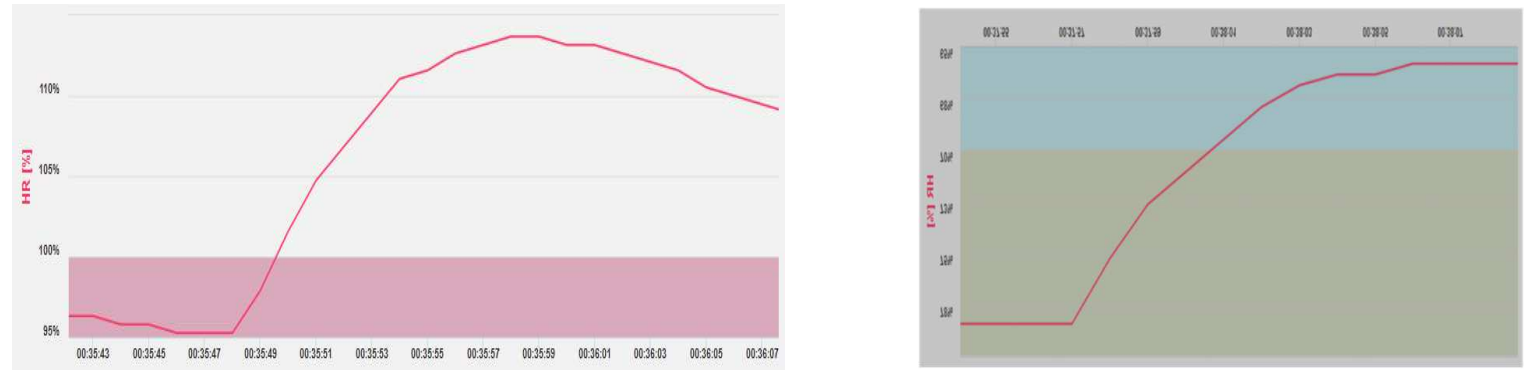

Figure 7: Illustrating the same images from Figure 5, but the one on the right has been inverted and rotated 180 degrees. This shows the angular and curvature similarities between the initiation and termination of the SVT occurrence

Along with the comparisons made of curvatures during SVT episodes, a search from other increases in HR (that were not abrupt departures from HRs of athlete performing similar exercises/drills at the same time as the subject) no instances of the aforementioned curvatures could be found. More exhaustive comparative analysis both with this subject's data set and investigation into other subjects with similar HR tracings would need to be accomplished before any sort of a more substantial hypothesis could be formulated. Currently, little to no other information about this observation could be found through the researched sources. 
The deviation noted on the daily HR trace that is decidedly similar between the initiation and termination can also be seen when analyzing the RRI. An immediate and abrupt departure and return to and from steady state can be seen on the RRI. Due to what is termed as smoothing of the data, between RRI and the HR trace, these departures appear with more of an angular appearance on the HR trace, but not nearly as abrupt as on the RRI. Given that the departures seen on the RRI are immediate in nature, a ramping up or somehow gradual increase of HR on the HR trace is a direct result of the "smoothing".

Further analysis was undertaken in the form of comparing the HR data, from what would be a daily analysis format, given in beats per min (BPM), to data presented as RRI. Part of the rational for this RRI analysis stems from a 2013 article by Wallot et al, that compared "the extent to which two standard heart beat measurements, the beat-to-beat interval (R-R interval) and the beats-per-minute (BPM), differ in the degree to which they contain information about the heart beat dynamics." (Wallot, Fusaroli, Tylén, \& Jegindø, 2013) To paraphrase their findings, a "smoothing" (removing noise from the data) can detract from the sensitivity of the final data presented meaning "a considerable detrimental effect of smoothing associated with BPM for the estimation of non-linear aspects of heart rate variability."(Wallot et al., 2013) With wanting to provide as much detail about the subject's particular occurrences, the BPM information provided for a good identification of when an occurrence was happening but more specific information was warranted.

Analysis of the RRI data was performed by using the identified occurrences from the BPM data and aligning those occurrences with the graphical representation of the RRI. This was all accomplished through the Polar Team Pro website. The RRI graphical representation display would appear inverse to the BPM display. This is due to the RRI being a measurement of time 
between heart beats, which would decrease as HR increases, and thus show as a "decrease" on the graph. Figure 8 gives a comparison of the BPM and RRI displays. For example it is evident that elevated HR around the 00:33:06 mark is mirrored by a reduction in RRI (Figure 8).

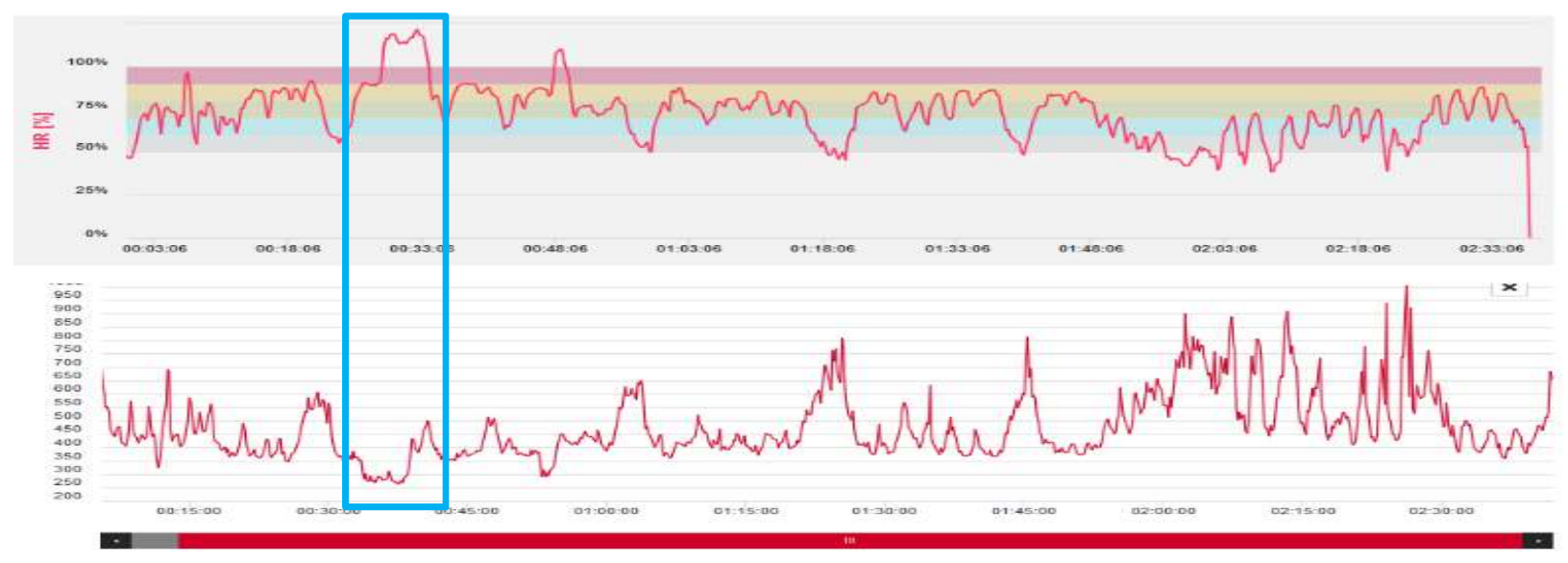

Figure 8: Showing a comparison between BPM (top) and RRI (bottom) and their inverse graphical relationship

Visual analysis or RRI data of the individual occurrences displayed an abrupt decrease in RRI to a new "steady-state" until termination of the occurrence (Figure 9). These rapid/abrupt departures were identified in almost all of the subject's RRI data that had an associated visually identified occurrence. There is one exception to this pattern that is explained later in this document. The exclusion of artifact occurrences can also be substantiated through the analysis of RRI data. 


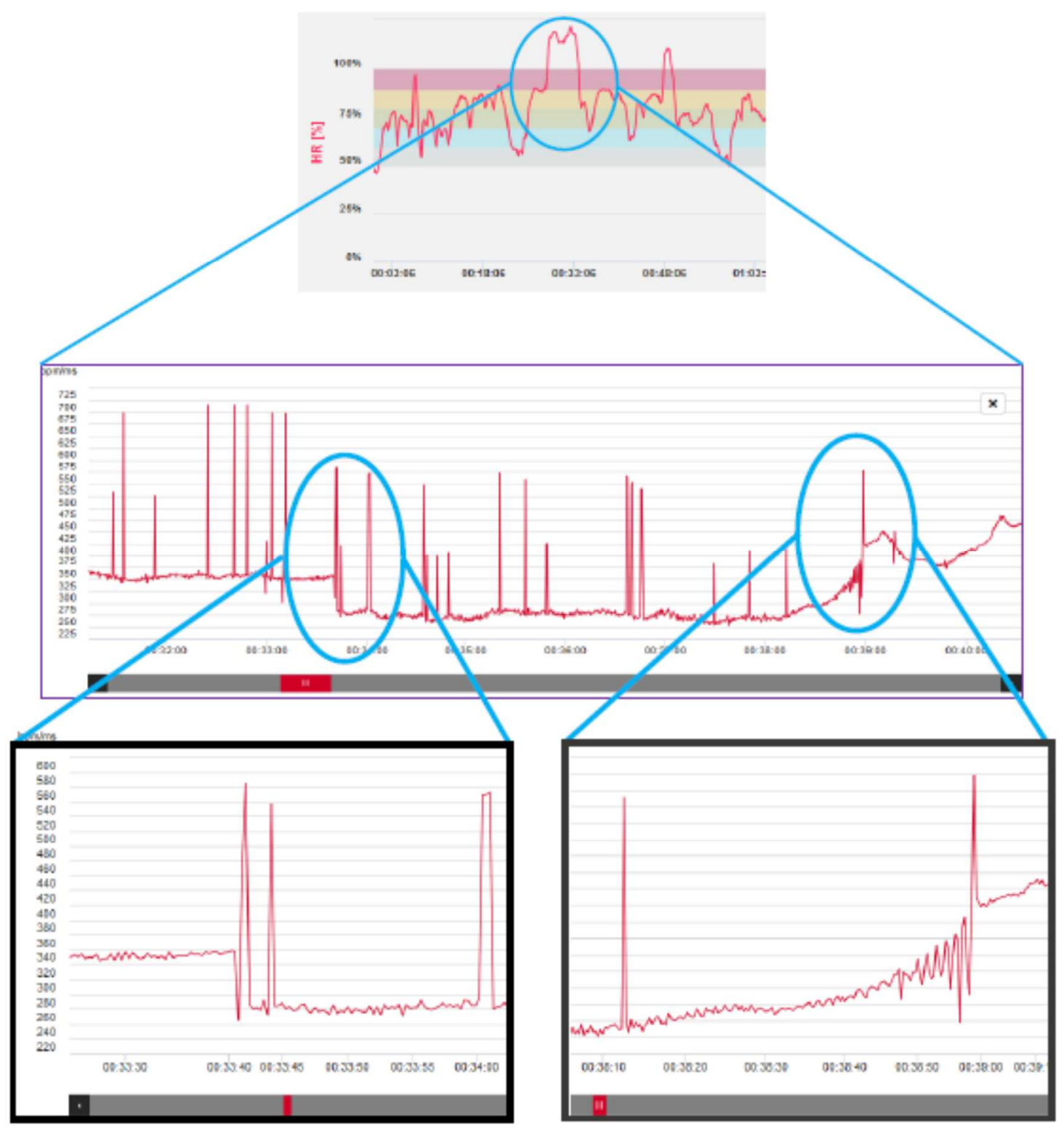

Figure 9: Illustrates close up of initiation and termination of occurrence as viewed in RRI format

Of particular note is that during the initiation of the occurrence, what would normally appear to be an artifact type deviation is noticed, after which RRI abruptly decreases in duration until the above mentioned steady-state is attained. Along with this, just prior to the termination of the occurrence, RRI can be seen to begin fluctuating more and more rapidly, almost a 
crescendo appearance, until an artifact type jump in RRI is seen. After this point RRI seems to return to a level that would be commensurate with the exercise/drill being performed (Figure 11).

During this analysis it was also discovered that all of the subject's occurrences did not always present with HRs in excess of $100 \%$. The arrow displayed on Figure 10 denotes just such an occurrence. Closer analysis of the HR tracing showed that initiation began at 131 BPM and had a maximum of 183 BPM, which are below the subject's maximum of 190 BPM. The RRI portion of the figure illustrates that the initiation and termination abrupt changes are present, as is the increasing magnitude of RRI just prior to termination. It should be noted that even though the HR tracing shows a sub-maximal HR occurrence, conversion of the RRI number to a HR indicates that it is actually a supra-maximal SVT occurrence with a peak of $228 \mathrm{bpm}$.
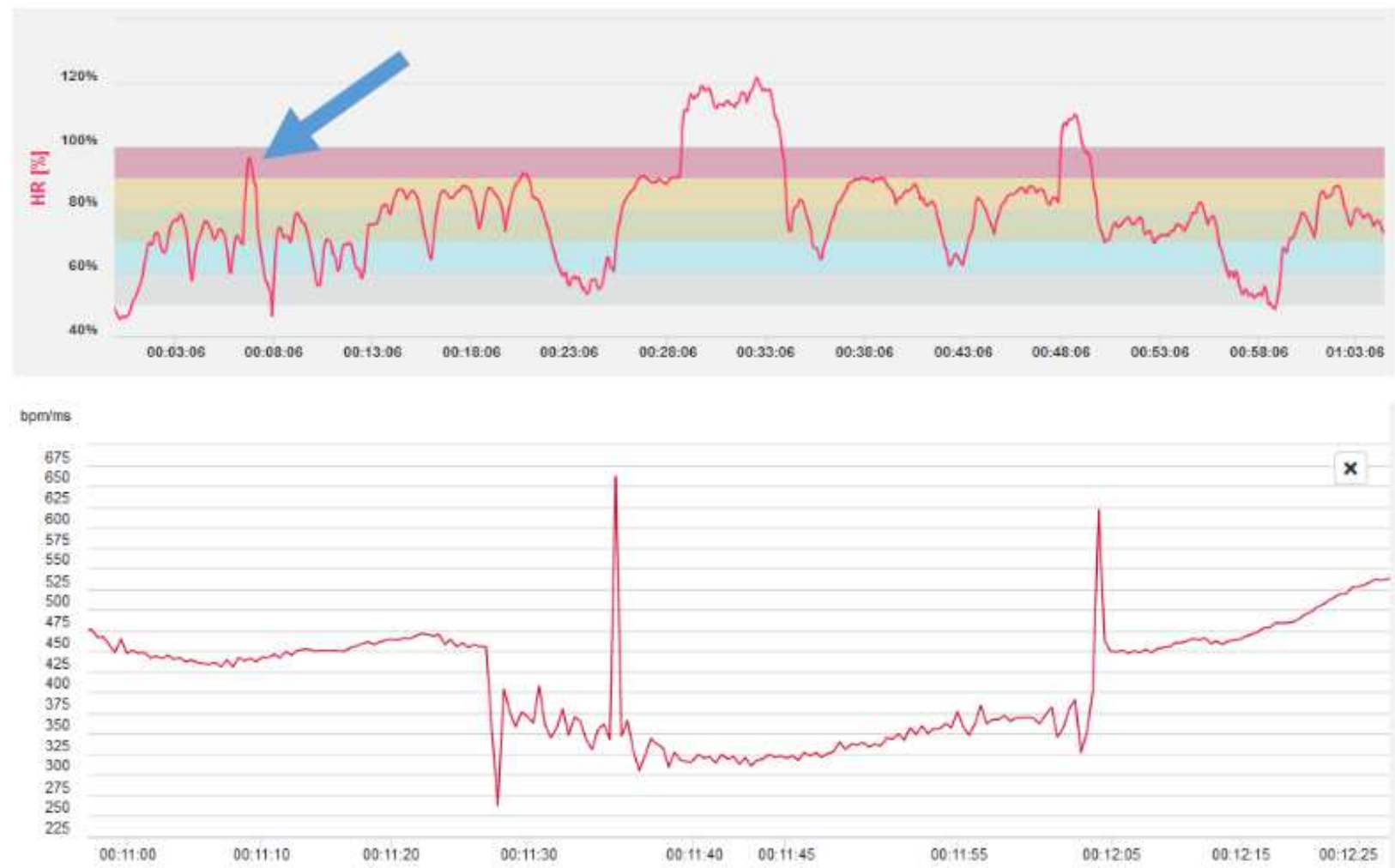

Figure 10: Illustrating RRI details of an occurrence taking place below the maximum calculated HR for the subject 
After analysis of all of the subject's recorded sessions, an additional 12 "sub-maximal" SVT occurrences. The term sub-maximal is used as these additional occurrences are not identifiable as exceeding the $100 \%$ calculated maximum HR for this athlete. The sub-maximal occurrences were only identified through review of the RRI data. Currently there are a total of 126 occurrences detected by RRI analysis as compared to an initial 97 occurrences through analysis of the BPM data provided.

Of these sub-maximal occurrences, all of them appeared to be below the subject's maximum HR of 190 on the HR tracings. Further calculations of all of the RRI information led to discovering that there were in fact no true sub-maximal occurrences. This led to investigation of the difference between values derived from HR tracings and RRI analysis. It was discovered that on average the RRI readings were 17 bpm higher than what was seen in the HR tracings. This difference would require further calculations and correlations to verify a consistent difference at all HR levels.

That being said, there is an instance that occurred that would appear to be a marked deviation from the other athletes and does have a sustained elevated HR. Figure 11 shows this occurrence in more detail. Of particular note is the RRI portion of the figure located at the bottom. The "normal" decrease in RRI, as seen in other occurrences is decidedly absent in this occurrence. 

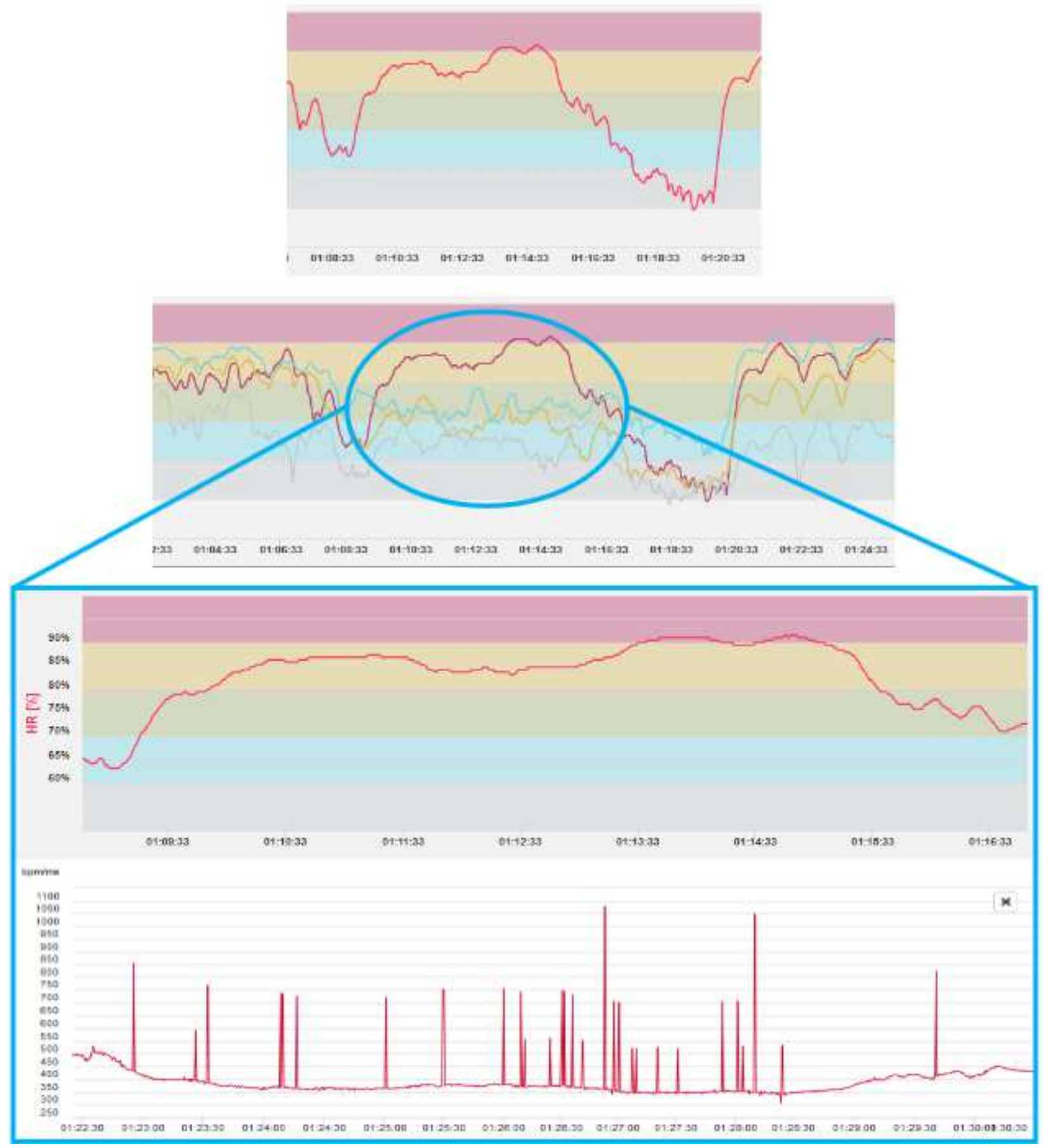

Figure 11: Illustrating elevated prolonged HR and subsequent RRI analysis

During inspection of the RRI information though there is no indication of occurrence. This particular occurrence lends support for not merely inspecting one portion of the data 
provided to make a determination as to the nature of the cardiac activity. In this instance, visual inspection would tend to lead to belief that a sub-maximal episode had occurred, with no corroborating data from the RRI it would be difficult to definitively identify this as an SVT event.

Additional measurements were ascertained to allow for identification of HR instances to be classified as either artifact or suspect. Initial analysis points to an instance that shows a rate increase $>10 \mathrm{bpm} / \mathrm{sec}$ or has a slope of $<0.1$ should be considered as artifact and thereby not considered for further analysis but instead addressing possible connectivity issues with the athlete/subject. Appendix D contains a complete listing of the measurements and how they were obtained and their utility. The data contained in Table 1 was obtained through analysis of all the sessions where the subject had an occurrence.

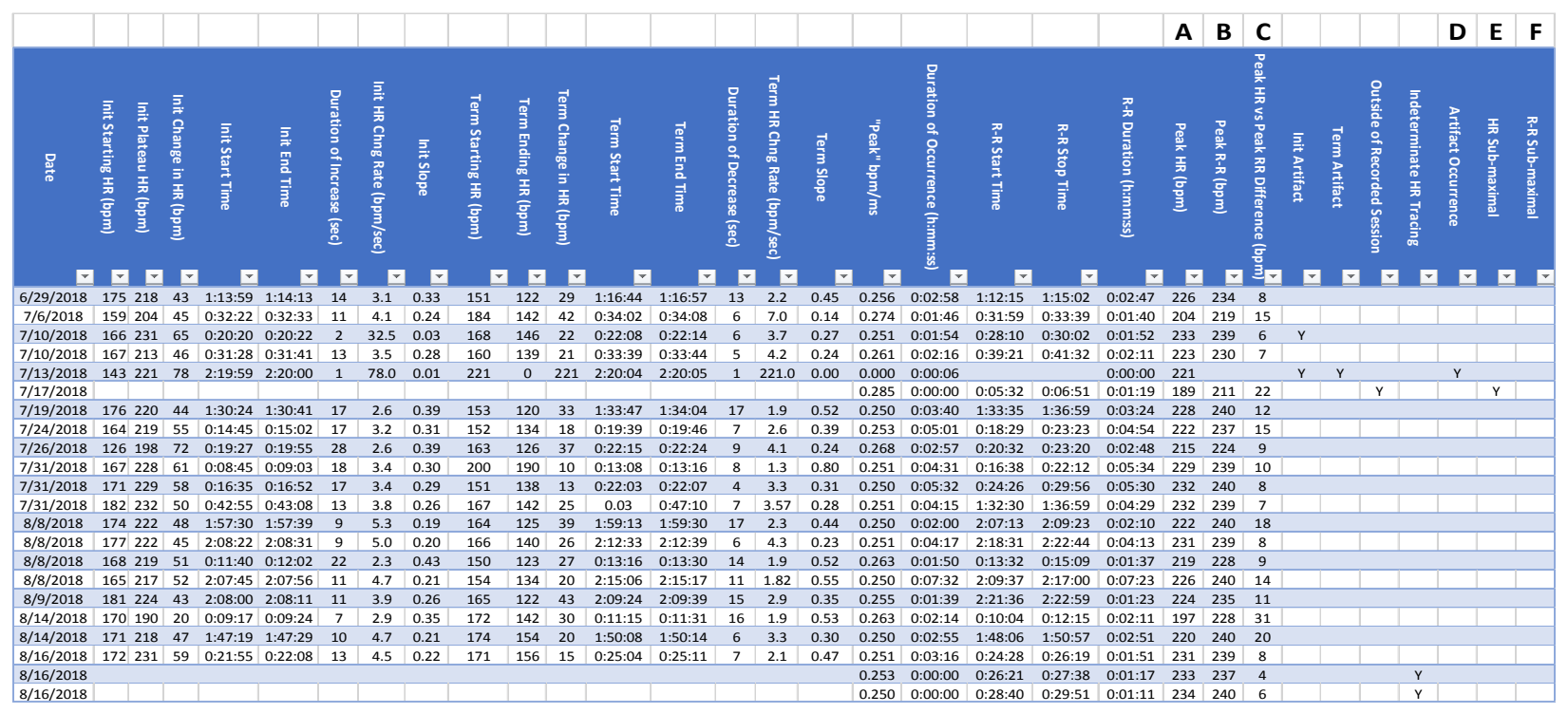

Figure 12: Numeric analysis of arrhythmia data taken from HR and RRI information (does not show all occurrences, complete table showing all occurrences is found in Appendix C) 
These occurrences were either seen in the HR tracings or under closer inspection of the RRI data. Once the data points were identified they were entered into their respective columns in a Microsoft Excel spreadsheet and ultimately used to calculate:
A.) Peak HR (as determined by HR tracing)
B.) Peak HR (as determined by RRI)
C.) Difference in HR Tracing Peak vs. RRI Peak
D.) Artifact Occurrence
E.) HR Submaximal Occurrence
F.) RRI Submaximal Occurrence

The listing above contains information that can act as a means of qualifying occurrences for inclusion or exclusion. The peak HR categories are included to identify a difference in data associated with the peak HR measurements and to indicate that a sub-maximal occurrence as observed on the HR tracing may in fact actually be a true supra-maximal occurrence depending on the numbers presented by the RRI data. If an occurrence is classified as a true artifact then it would be excluded from further analysis. Table 2 contains more specific statistical values.

Table 1: Occurrence statistical values

\begin{tabular}{ccccc}
\hline Variable & Minimum & Maximum & Average & Standard Deviation \\
\hline HR Duration & $0: 00: 14$ & $0: 26: 29$ & $0: 03: 43$ & $0: 03: 47$ \\
RR Duration & $0: 00: 05$ & $0: 26: 25$ & $0: 03: 33$ & $0: 03: 45$ \\
HR Peak & $156 \mathrm{bpm}$ & $238 \mathrm{bpm}$ & $215 \mathrm{bpm}$ & $17.5 \mathrm{bpm}$ \\
RR Peak & $197 \mathrm{bpm}$ & $240 \mathrm{bpm}$ & $233 \mathrm{bpm}$ & $10 \mathrm{bpm}$ \\
HR vs RR Peak Diff & $1 \mathrm{bpm}$ & $63 \mathrm{bpm}$ & $17 \mathrm{bpm}$ & $13 \mathrm{bpm}$ \\
Rate Increase & $0.4 \mathrm{bpm} / \mathrm{s}$ & $7.7 \mathrm{bpm} / \mathrm{s}$ & $3.3 \mathrm{bpm} / \mathrm{s}$ & $1.3 \mathrm{bpm}$ \\
Initiation HR & $123 \mathrm{bpm}$ & $188 \mathrm{bpm}$ & $162 \mathrm{bpm}$ & $22 \mathrm{bpm}$ \\
\hline
\end{tabular}


In a study by Maurer et al., approximately $6 \%$ of the 1383 subjects tested evidenced exercise induced SVT. In comparison with this case study though, the majority of the recorded SVT episodes that were identified were only 3-5 beats in length with and were rarely in excess of 30 seconds in length. As can be seen in table 2, the average duration of this subject is calculated to be $0: 03: 33$. The majority, $96 \%$ of the subjects in the study by Maurer, were in fact asymptomatic, much like the subject of this case study (Maurer, Shefrin, \& Fleg, 1995). According to the Mayo Clinic some of the clinical symptoms of SVT could include, but are not limited to:

- A fluttering in your chest

- Rapid heartbeat (palpitations)

- Shortness of breath

- Lightheadedness or dizziness

- Sweating

- A pounding sensation in the neck

- Fainting (syncope) or near fainting

("Supraventricular tachycardia - Symptoms and causes," n.d.). The subject of this case did in fact respond that she did experience the sensation of a rapid heartbeat, or palpitation, but this was only after she was "identified" with the use of HRM data. In other words, the sensation that she was experiencing were to be considered "normal" to her as she was unaware that there was in fact anything wrong with what she had always been experiencing.

\section{Expanded Screening}

This system is also utilized for 3 other sports teams for the same purpose. During this time the data previously collected on the other sports teams monitored with the previously mentioned system were also screened. A cut-point of $205 \mathrm{bpm}$ was used for screening purposes. This value was to account for the fact that athletes, when giving a maximal effort, are prone to 
exceeding their predicted maximum HR. A total of 64 athletes across 4 teams were screened to identify HRs that were remaining elevated in a fashion that could indicate a tachycardia type arrhythmia.

This screening process consisted of both the visual inspection for marked deviations from other athletes performing the same type of drill as well as using downloaded data to identify athletes exceeding the $205 \mathrm{bpm}$ cut-point. The teams varied in the number of players, length of season, number of sessions recorded, and type of sport. This would account for the varying number of identified instances between the four teams.

Table 2: Number of instances in excess of $205 \mathrm{bpm}$

\begin{tabular}{cc}
\hline Team & Number of Instances \\
\hline Women's Volleyball (WVB) & 326 \\
Women's Soccer (WSOC) & 848 \\
Women's Basketball (WBB) & 202 \\
Men's Basketball (MBB) & 835 \\
\hline
\end{tabular}

An artifact occurrence takes place when there is intermittent contact between the conductive material of the chest strap and the athlete's bare skin. Another scenario is that some sort of mechanical anomaly is occurring where the monitor connects to the strap. In either case a distinct difference in data collected occurs when these phenomena take place.

In a 2016 article, HR artifact is defined as "A step increase or decrease in HR to a stable new state and a step decrease back to the same state with no change in other variables... This signal usually generates a new plateau, causing a square wave pattern” (Hravnak et al., 2016). In many of the instances with the analysis that was undertaken this steep increase or decrease was observed. This definition coupled with the calculated rate changes and slopes were used as criteria to evaluate occurrences that met the $>205$ bpm cutoff. 
To summarize a similar case study published in 2013, Mussigbrodt et. al., presented 3 cases that were identified as symptomatic of palpitations. Researchers used recordings from their HRMs to compare results gained by testing with either an ECG or invasive electrophysiological (EP) study. These comparisons were done in an attempt to provide evidence that a symptomatic subject with HR monitor information that displays some sort of anomaly can be useful in the identification of arrhythmia causes. Their conclusions from the three cases presented moved towards pointing out the "utility of HR monitors with chest strap transmitters in diagnosing exercise-induced arrhythmias and therefore prompting appropriate, timely treatment." (Müssigbrodt et.al. 2013)

In contrast to the previously mentioned study, the case currently presented is with a subject that essentially was asymptomatic. Through analysis of the HRM system data provided, a determination was made to approach the athlete with questions about her observations during specific sections of a recorded session. Once the subject relayed information that would indicate some sort of symptomatic response a determination was made to pursue further investigation of a possible arrhythmia. Had the initial suspect session not been noticed and analyzed it is unlikely that either data analytics, coaching staff, or the athlete/subject would have been made aware of the presence of any sort of arrhythmia.

During an interview with the subject an overall lack of "symptoms" became apparent. Subject stated that there is no real feeling or sensation during an occurrence while performing a drill. More to the point there is a noticeable difference in recovery meaning that "when the drill is over I sometimes feel that my heart is racing or pounding when it should be slowing down." Subject was also asked if anything was ever said to anyone about these feelings/sensations. Subject relayed that "no, I never mentioned it to anyone". When asked as to why not, an answer 
of "I didn't want to be taken out of play, I guess that is a typical athlete answer" was given. Family history was also explored and it was clarified that subject's great-grandfather and grandfather on mother's side both had heart attacks and died during their early to mid 50's, with the grandfather having two and subsequently dying from the second.

These questions do shed some light on possible pre-determining factors associated with the diagnosis of SVT. Additionally, awareness of symptoms would play a large part in helping to identify athletes/students with possible arrhythmias. Educating both staff and participants about what to look for or be aware of should be part of a screening process. One of the more notable items is that the athlete/student may be reluctant to bring any such symptoms to light in fear of not being able to participate. Currently the subject has been diagnosed with exercise induced SVT and is scheduled to have an ablation within the next month. A more exact diagnosis may be available at that time but those details are not presently available.

In the study by Gajda, et.al, a flow chart is presented for recommended sequence of addressing concerns associated with arrhythmias identified through the use of HRMs (Figure 15). For all intents and purposes our process was similar in seeking an appropriate course of action to address the presence of a possible condition. A variable that is not mentioned is the frequency or number of occurrences over a given period of time. In the case of the subject for this study, there were in fact multiple occurrences of suspect anomaly over a 10 month period. The analysis of HR data served as guidance in identify suspect occurrences but was not used as a diagnosing instrument. 


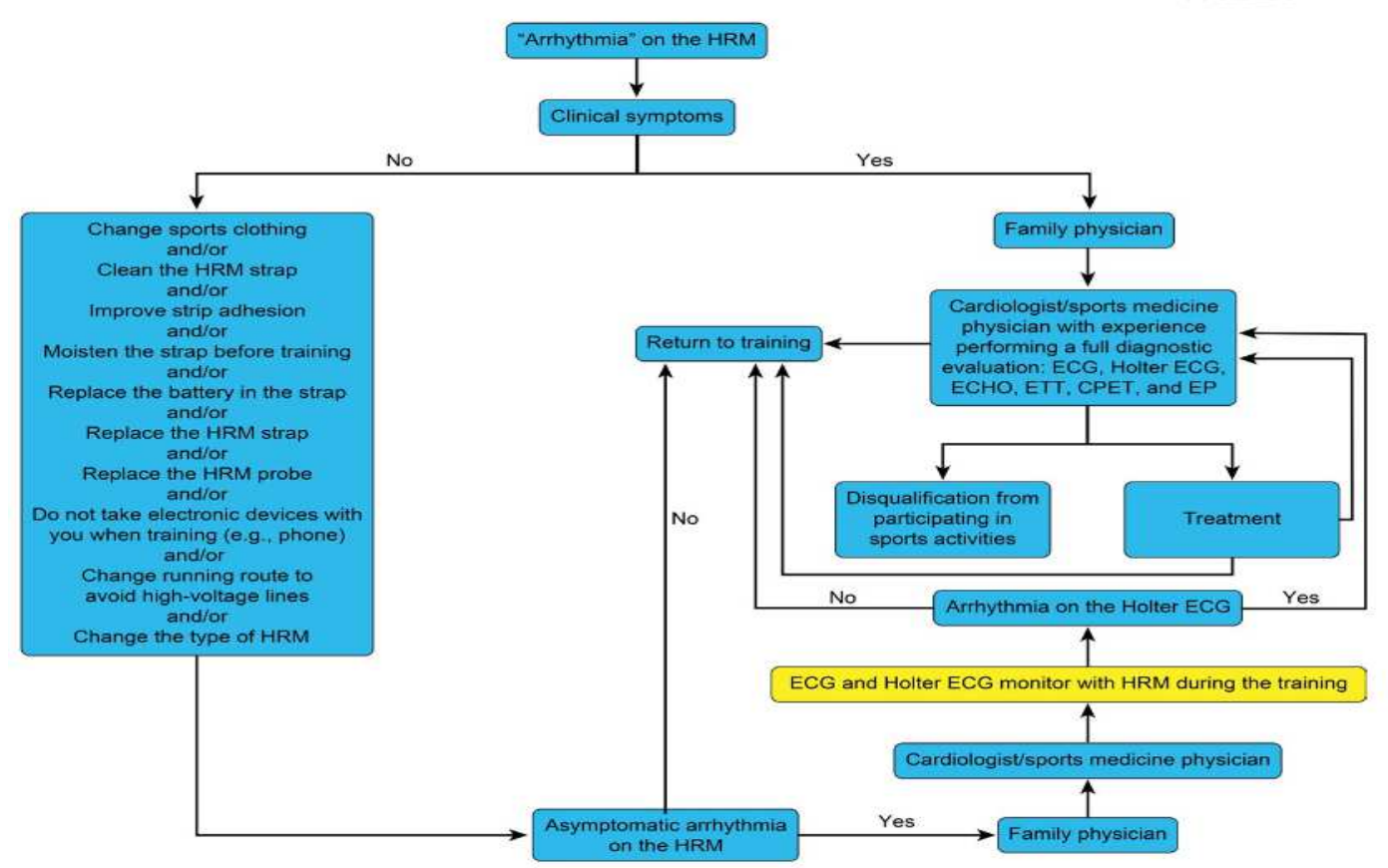

Figure 13: Showing copy of flow chart from article by Gajda, et al. (Gajda et al., 2018a), that displays a recommended sequence with arrhythmia is detected using HRM

Taking the number of occurrences into consideration should not be used as a sole cutpoint for pursuing clarification. If previously recorded data is available for the athlete or individual in question, analysis of both the HR tracings and RRI information can assist in providing guidance. At no time should HRM data be used as a sole method of determining or diagnosing any sort of medical condition.

With the varying methods of analysis used to determine the presence of an occurrence, care must be taken to not simply rely on one form of analysis. Visual screening of the HR tracing can prove useful to identify a possible trend and is a relatively quick process. RRI information proves extremely valuable to provide support for what is gleaned from the visual inspection of the HR tracing. Use of the two different outputs together provides a strong case for identifying if an instance requires elevation. 


\section{Conclusions}

With the ever increasing need for performance evaluation of athletes, or activity monitoring of students in general, an opportunity to identify a possible arrhythmia may present itself. In this case, an asymptomatic athlete was identified during what would otherwise be routine analytics of HR data for the purposes of providing improved performance information about the condition of the athletes on a given team. During this analysis it was noticed that there was a recurrence of sustained elevated HR episodes. Due to the frequency of marked departures it was decided to elevate the information to coaching staff for determination of course of action. With the athlete's health and wellness being paramount, a closer physical evaluation by appropriate medical personnel resulted in the identification of what is termed as exercise induced supraventricular tachycardia.

The level of analysis required to use this technology for initial screening of a possible arrhythmia should not be based on solely reviewing daily outputs. To avoid un-necessary testing of a subject, and possibly causing un-needed stress in the process, further analysis to include review of RRI data should be accomplished. Ultimately a system, process, or policy could be developed to appropriately elevate individuals that have been evidencing some sort of arrhythmia indicating data even though they themselves may present as either symptomatic or asymptomatic. More stringent criteria for identifying artifact anomalies would be very beneficial to prevent a flood of "false-positive" instances. This would require knowledge of identifying the differences between an artifact occurrence and an occurrence that may in fact be truly suspect. More research would be required to more accurately define what would constitute artifact versus suspect. 
Although not as specific or diagnostically capable as either an ECG or Holter monitor, HRM data analytics can be used to identify recorded data indicative of an arrhythmia while the individual may have remained otherwise asymptomatic (either due to not knowing they are experiencing symptoms or being genuinely asymptomatic). Even if the probability of the athlete being diagnosed with some sort of actual arrhythmia is small, the benefits of detection through HRM outputs being elevated to the appropriate levels far outweigh the possible loss if not identified and pursued. 


\section{CHAPTER II: REVIEW OF THE LITERATURE}

Currently in the pursuit of fitness and overall well-being there seems to be an endless supply of wearable technology. This technology can range from the standard heart rate monitor to devices that will measure variables that until a few year ago required a trip to a laboratory and possibly a somewhat invasive procedure. With all of that being said the need for information keeps on growing. Aside from the numbers of devices and what they can measure, how the measured items are analyzed is also expanding. With a few calculations, what was seemingly simple information can be turned into something even more valuable. What happens though when a possible source of information is overlooked, either because it is not noticed, not understood, or not viewed as reliable when compared with other methods of measurement.

The variable in question is the one that was mentioned first, heart rate (HR), and the possibility that it could be used to screen or identify individuals with conditions beyond just being "unfit". HR monitors usually have the means to provide the user with a graphical representation of their HR as an exercise session, practice, or game has progressed. With proper analysis this graphical representation can be converted into information that provides the user about how they can improve their performance. It can also be used to identify some cardiac irregularities. There is some debate as to the reliability of this interpretation as it compares to other more clinical means of evaluating the possible presence of cardiac abnormalities.

In this review of literature, the main focus will be on the existing studies that are available and how they relate to the use of HR monitor outputs in determining the presence of arrhythmias compared with other modes. Along with that the overall view that the researchers have about using HR monitors in this fashion. The comparison of subjects used in these studies will also be addressed. 
A 2000 study by Boudet and Chamoux tested the ability of three different HR monitors (Accurex Plus, Vantage NV, and PE 4000) to detect and report abnormal heart rhythms and arrhythmias. This study was done with the intent of comparing the accuracy of "newer" technology to the accuracy of a previous generation technology HR monitor. These different HR monitors were compared to the output of an electrocardiogram (ECG) simulator in a controlled laboratory setting.

The three devices tested produced recorded values that were between 89 and $98 \%$ accurate with steady state measurement. When measuring changes in HR "In transient phases HRM's are never accurate.’(Boudet \& Chamoux, 2000). Of note in this study is that the authors mention, in brief, a case where an individual had noticed a manifestation of decreased HR during exercise. Upon seeking medical care for clarification and presenting the HR output values, the individual was further screened and diagnosed with a "severe cardiac disorder and led to the implantation of a pacemaker.”(Boudet \& Chamoux, 2000).

This study highlights the accuracy of HR monitors when compared to each other and how accurate they can be in a laboratory setting. This study does not address what can happen in actual exercise settings and only focuses on a controlled setting. Of note, this article was published in 2000 and there have since been advances in technology that could have improved the ability of HR monitors to adapt to changes in HR in a smoother fashion and provide more reliable monitoring of changes.

In 2017, Gajda, Biernacka, and Drygas tested 142 endurance athletes with suspect arrhythmias. The subjects had either self-referred themselves or were referred by medical practitioners due to abnormalities noticed in their outputs from HR monitors. During testing all 
of the participants were fitted with their HR monitor (the type worn during the recorded abnormality) and a Holter monitor. Subjects were to perform a monitored training plan similar to the one performed when the abnormality was recorded on their HR monitor.

After analysis it was discovered that $99.3 \%$ of the subjects $(\mathrm{n}=141)$ displayed either no recorded abnormalities, abnormalities recorded on the HR monitor and not the Holter monitor, or a single ectopic beat recorded by either the HR monitor or Holter, but not both. $0.3 \%$ of the subjects ( $\mathrm{n}=1)$ did report symptoms (decreased performance, with palpitations), and indicated an arrhythmia recorded on both HR monitor and Holter monitor.

One of their conclusions is that "The value of HRMs for detecting arrhythmias in runners and cyclists is not supported by the results of this study. This study has shown that "arrhythmias" measured by HRMs (an unexpected increase in HR measured by the HRMs) during typical endurance training in asymptomatic athletes, cyclists, and runners, is an artifact"(Gajda, Biernacka, \& Drygas, 2018a). The researchers do not totally discredit the usability of HRMs but in fact err on the side of caution to prevent too much weight being put on use of the HRM output to be used as a diagnostic tool.

A couple of interesting notes with this study is that there is no actual quantification of what would be considered artifact. More to the point it is just stated that artifact is a result of some sort of connectivity or mechanical defect. In addition to that, all of the athletes studied participate in endurance activities and for all intents and purposes are physically asymptomatic.

A 2017 case-study by Thabouillot et al, outlines a 45 year old male who presented with symptoms during a $10 \mathrm{~km}$ run/race. These symptoms included, difficulties maintaining his usual race pace, complete amnesia and difficulties walking during the last $1 \mathrm{~km}$ and eventually losing consciousness (Thabouillot et al., 2017). The subject was wearing a HRM and the information 
recorded during the race was used to reveal that a tachycardic event had taken place. This led to further testing which included a stress test and then application of a Holter monitor. During both tests the subject's own HRM was worn for comparison. In summary, the subject was diagnosed with "adrenergic paroxysmal atrial fibrillation (AF)...(Thabouillot et al., 2017)

The aforementioned case-study demonstrates support that HRMs can be used to assist with the discovery of arrhythmia but in and of itself should not be used to diagnose an arrhythmia. The authors clearly advocate for use of the information but caution at the same time by saying "The precision of these devices remains open to debate due to the fact that they have never been independently tested, and also because there exists a reference technique... (Thabouillot et al., 2017). 


\section{REFERENCES}

Boudet, G., \& Chamoux, A. (2000). Heart Rate Monitors And Abnormal Heart Rhythm Detection. Archives of Physiology and Biochemistry, 108(4), 371-379. https://doi.org/10.1076/apab.108.4.371.4304

Gajda, R., Biernacka, E. K., \& Drygas, W. (2018). Are heart rate monitors valuable tools for diagnosing arrhythmias in endurance athletes? Scandinavian Journal of Medicine \& Science in Sports, 28(2), 496-516. https://doi.org/10.1111/sms.12917

Helton, M. R. (2015). Diagnosis and Management of Common Types of Supraventricular Tachycardia. American Family Physician, 92(9), 793-800.

Hravnak, M., Chen, L., Dubrawski, A., Bose, E., Clermont, G., \& Pinsky, M. R. (2016). Real Alerts and Artifact Classification in Archived Multi-signal Vital Sign Monitoring DataImplications for Mining Big Data — Implications for Mining Big Data. Journal of Clinical Monitoring and Computing, 30(6), 875-888. https://doi.org/10.1007/s10877015-9788-2

Maurer, M. S., Shefrin, E. A., \& Fleg, J. L. (1995). Prevalence and prognostic significance of exercise-induced supraventricular tachycardia in apparently healthy volunteers. The American Journal of Cardiology, 75(12), 788-792.

Müssigbrodt, A., Richter, S., Wetzel, U., Van Belle, Y., Bollmann, A., \& Hindricks, G. (2013). Diagnosis of Arrhythmias in Athletes Using Leadless, Ambulatory HR Monitors. Medicine \& Science in Sports \& Exercise, 45(8), 1431. https://doi.org/10.1249/MSS.0b013e31828ca1bf 
Supraventricular tachycardia - Symptoms and causes. (n.d.-a). Retrieved February 14, 2019, from https://www.mayoclinic.org/diseases-conditions/supraventriculartachycardia/symptoms-causes/syc-20355243

Thabouillot, O., Bostanci, K., Bouvier, F., Dumitrescu, N., Stéfuriac, M., Paule, P., \& Roche, N.C. (2017). Syncope During Competitive Events: Interrogating Heart Rate Monitor Watches May Be Useful! Prehospital and Disaster Medicine, 32(6), 691-693. https://doi.org/10.1017/S1049023X17006896

Wallot, S., Fusaroli, R., Tylén, K., \& Jegindø, E.-M. (2013). Using complexity metrics with R-R intervals and BPM heart rate measures. Frontiers in Physiology, 4. https://doi.org/10.3389/fphys.2013.00211 


\section{APPENDIX A: COMPOSITE/DESCRIPTIVE IMAGES}

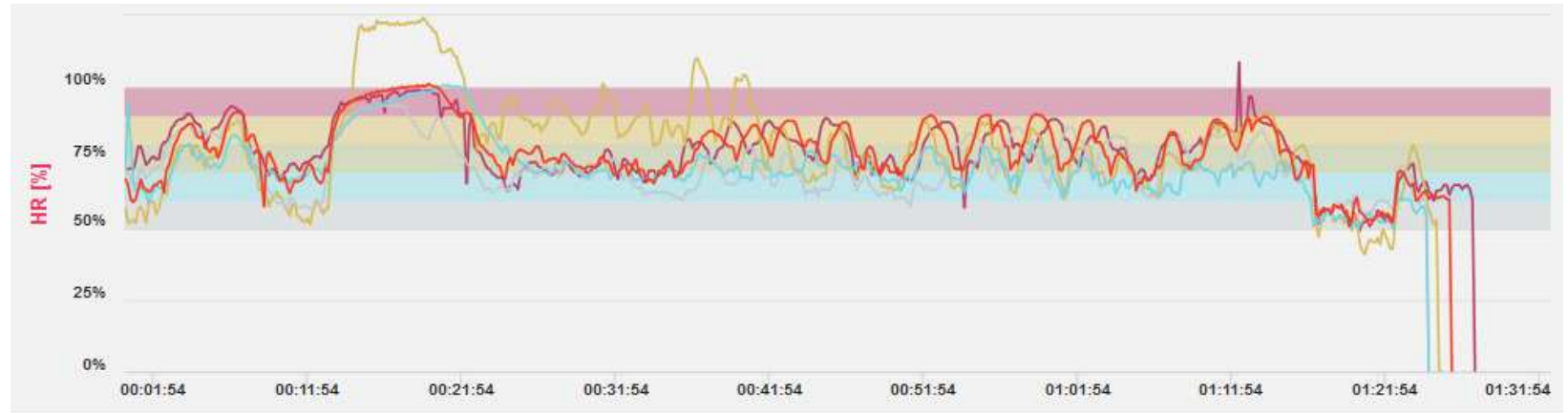

Figure A-1: Session recorded on 1/18/2019, showing departure of subjects HR from other athletes

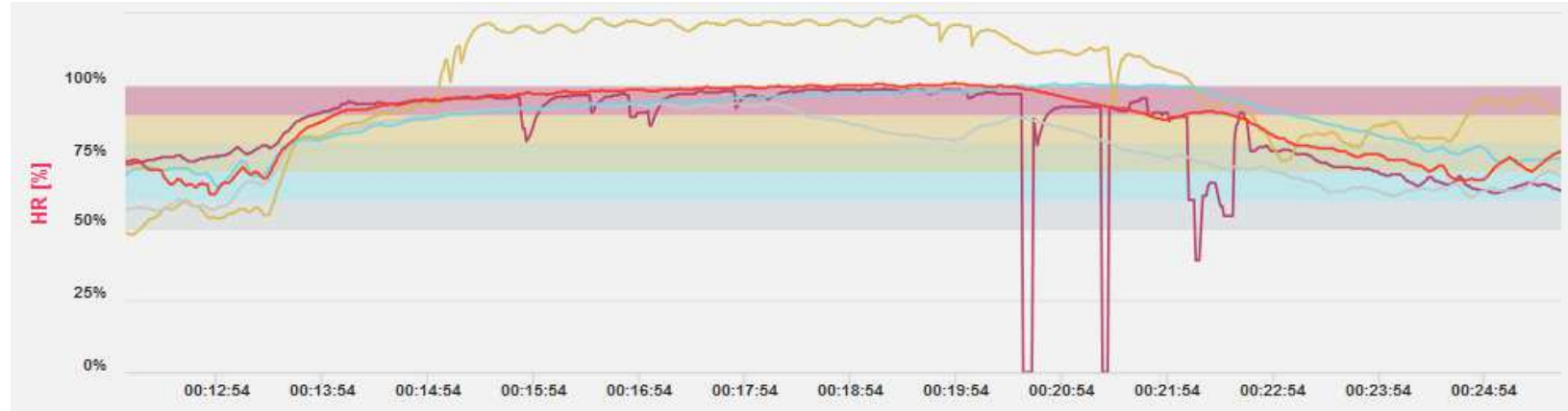

Figure A-2: Close up of Figure A-1, showing that subjects HR continues to fluctuate with activity changes despite elevation

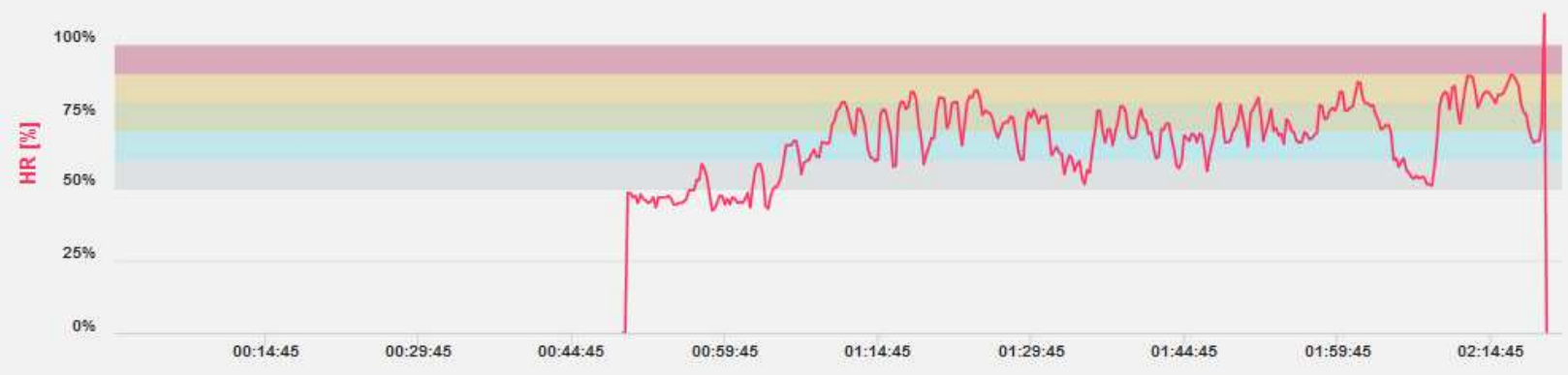

Figure A-3: Recorded on 7/13/2018 


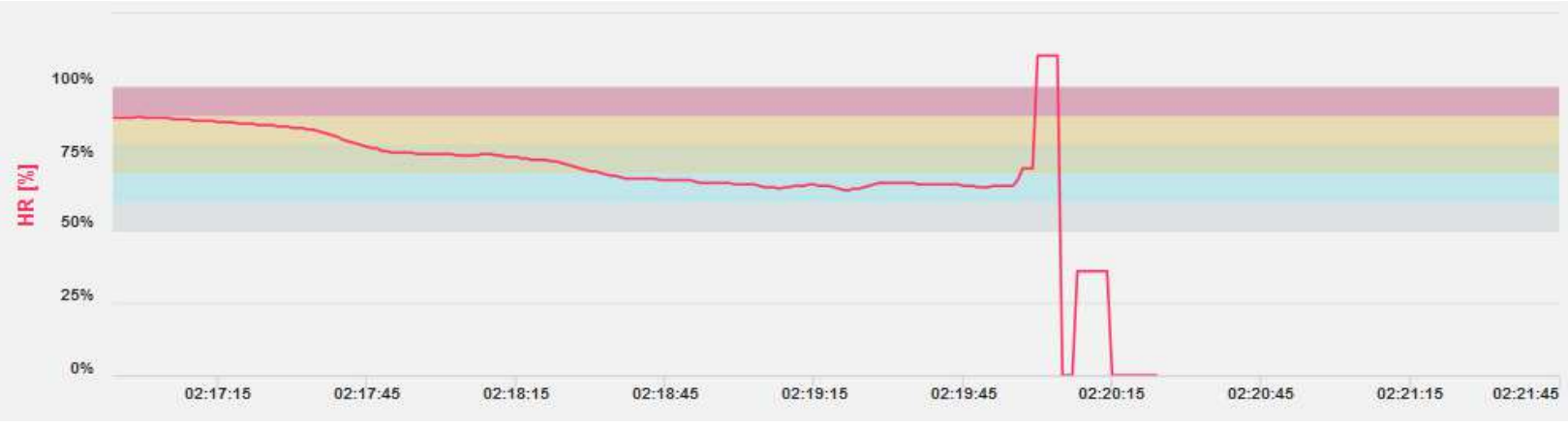

Figure A-4: Close-up of figure A-3, note near-vertical increase and decrease of HR and angular leveling of HR, indicative of artifact.

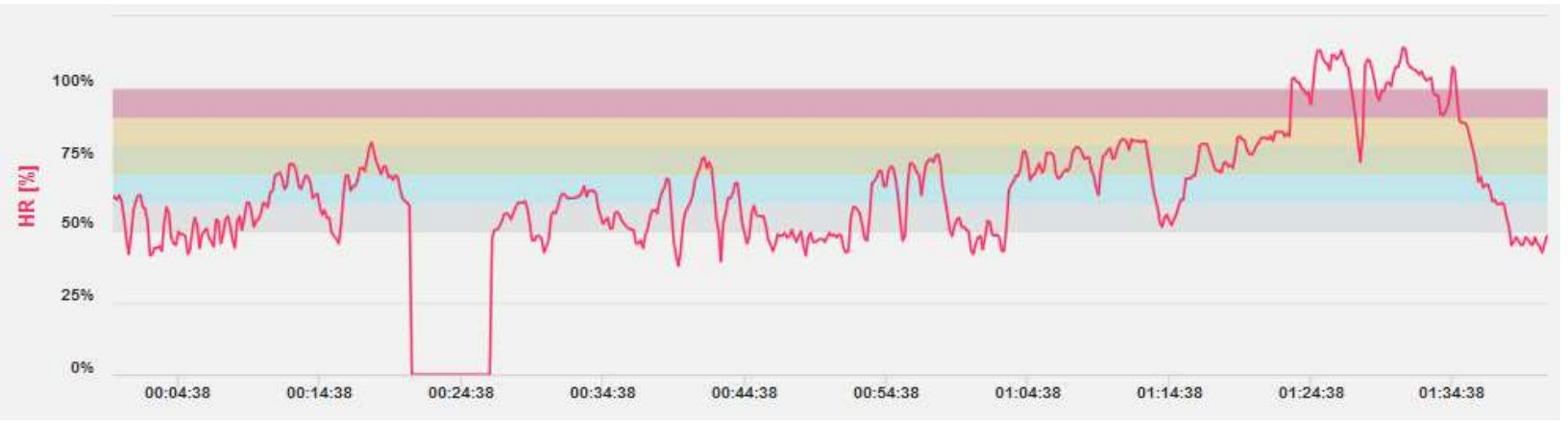

Figure A-5: 11/7/2018, session lasting 01:41:22

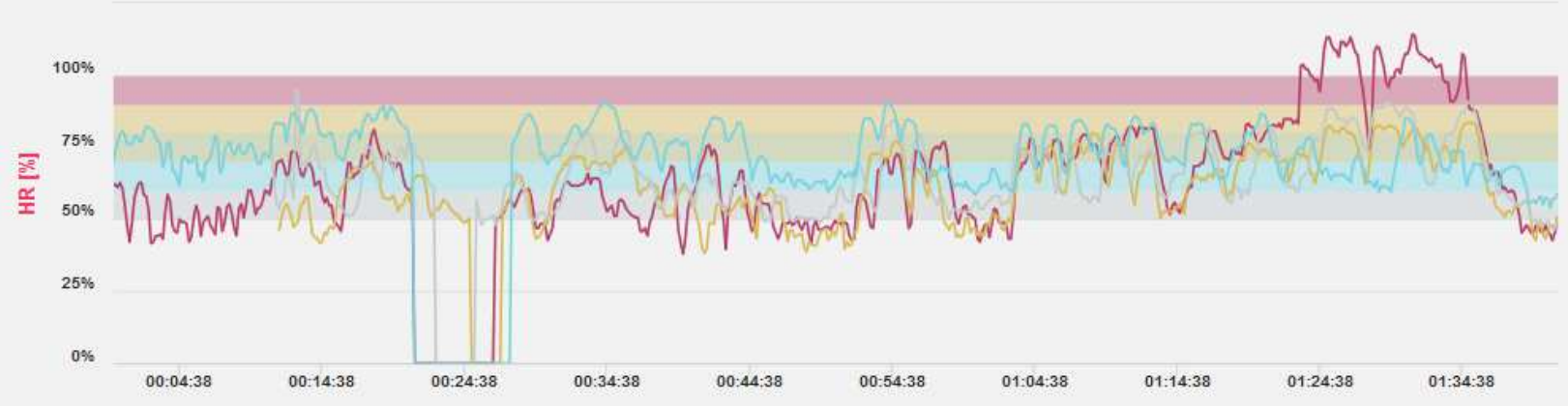

Figure A-6: Same session as from figure A-5 but with added tracings from other athletes to show departure. 


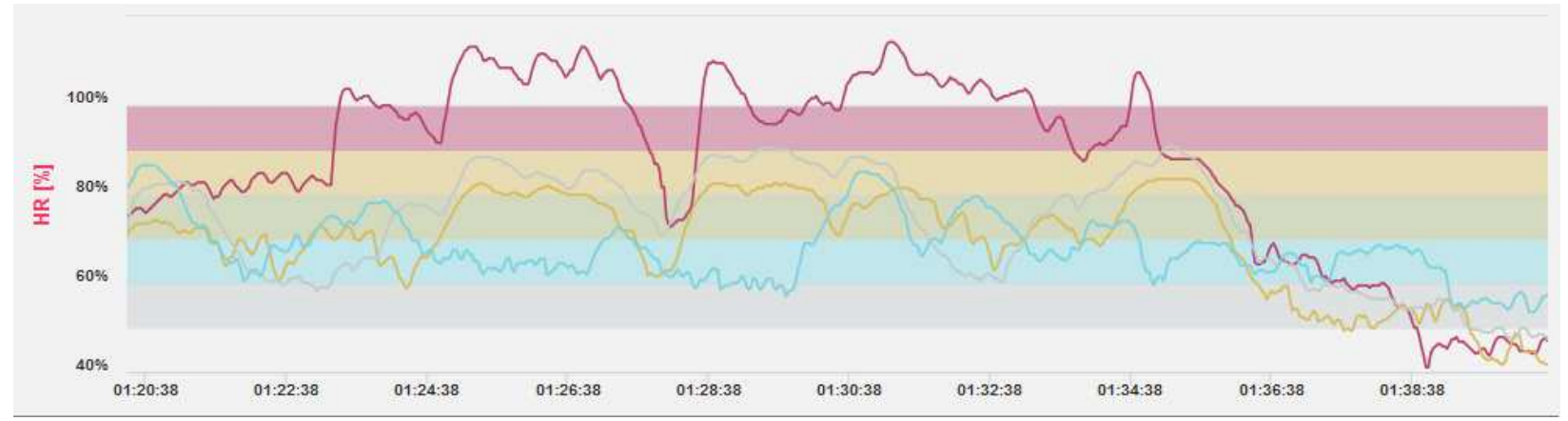

Figure A-7: Close-up of figure A-6.

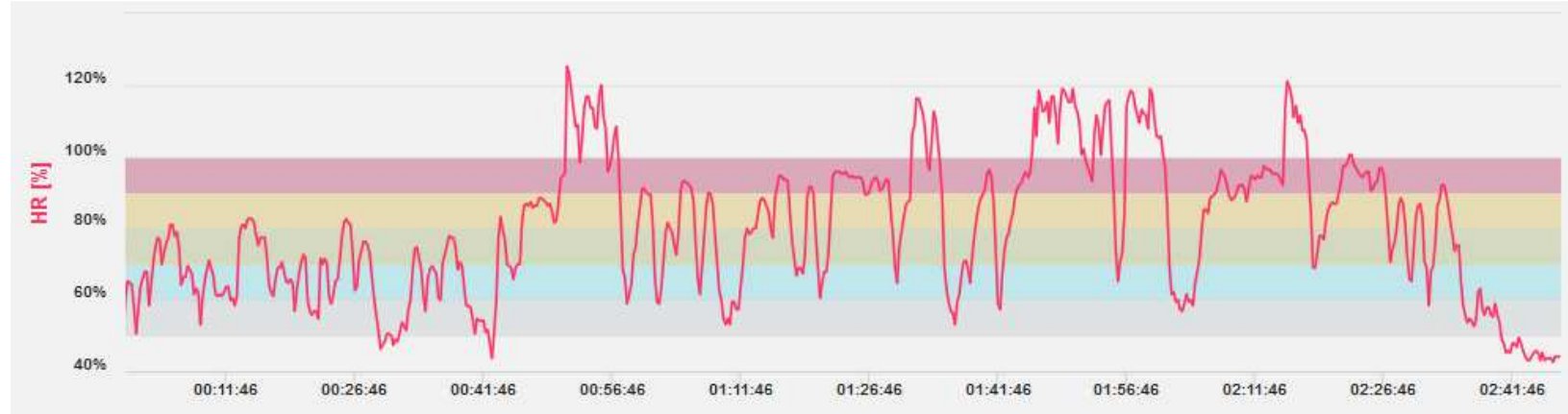

Figure A-8: 10/15/2018, With what appears to be 5 occurrences, the first occurrence at approximately 00:51:00 does not have a reliable initiation point and actually meets criteria for artifact, but does have a clear ending at 00:57:54, second occurrence lasted 00:01:22 peak of 221, the third occurrence began at approximately 01:44:00 but had some artifact at the initiation and midway through. The last occurrence had a duration of 00:03:18 peak of 230. RRI provides for 5 definitive occurrences.

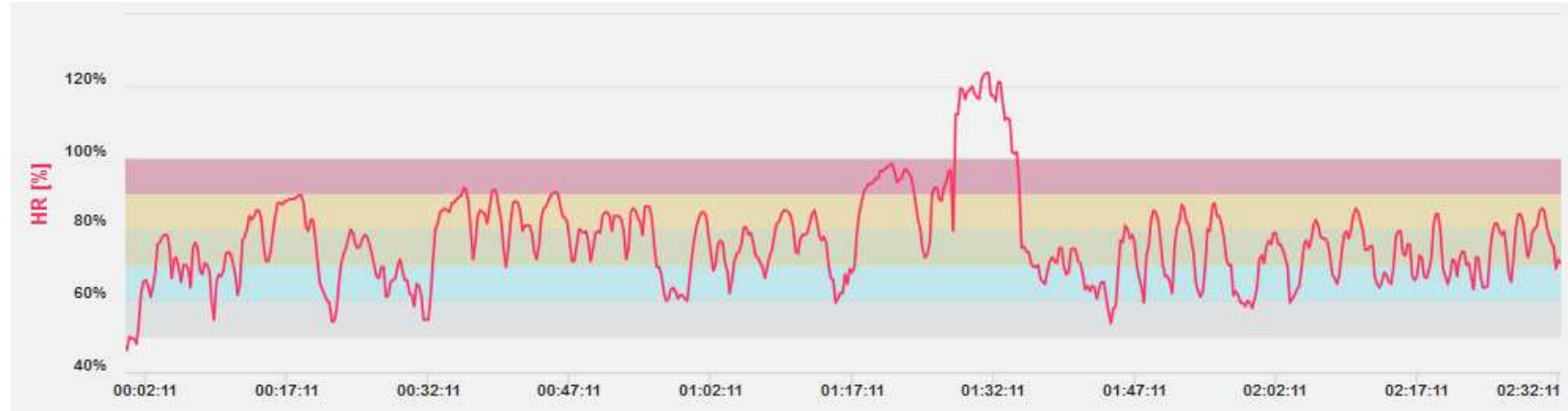

Figure A-9: 2/4/2019, session lasting 02:32:29, 1 occurrence lasting 00:06:53 peak of 235 . 


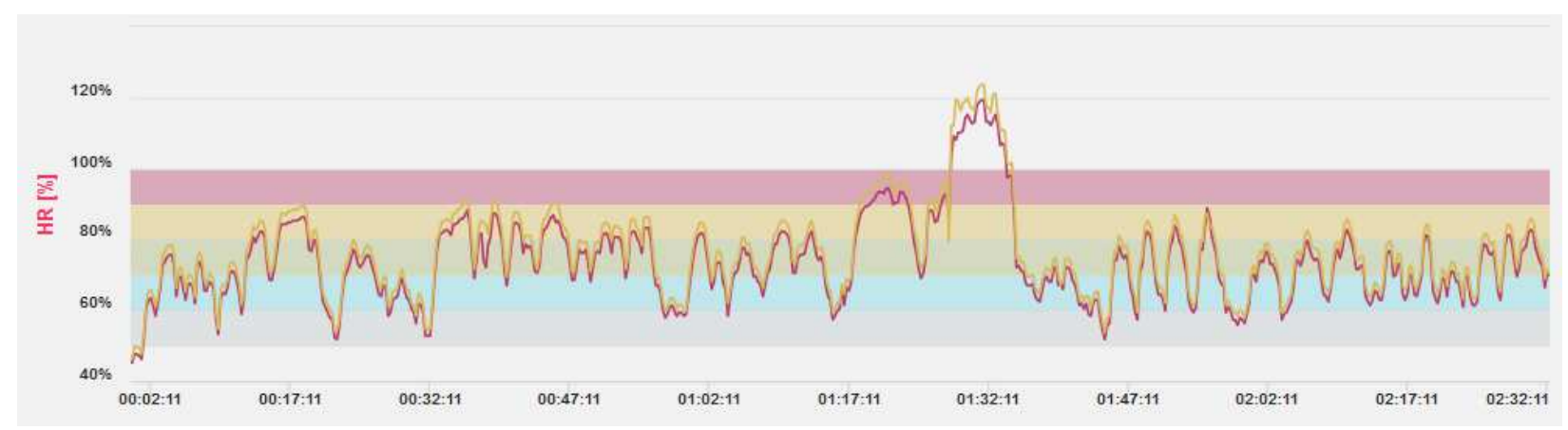

Figure A-10: This image is to show that there were running 2 sensors simultaneously to rule out possibility of equipment error.

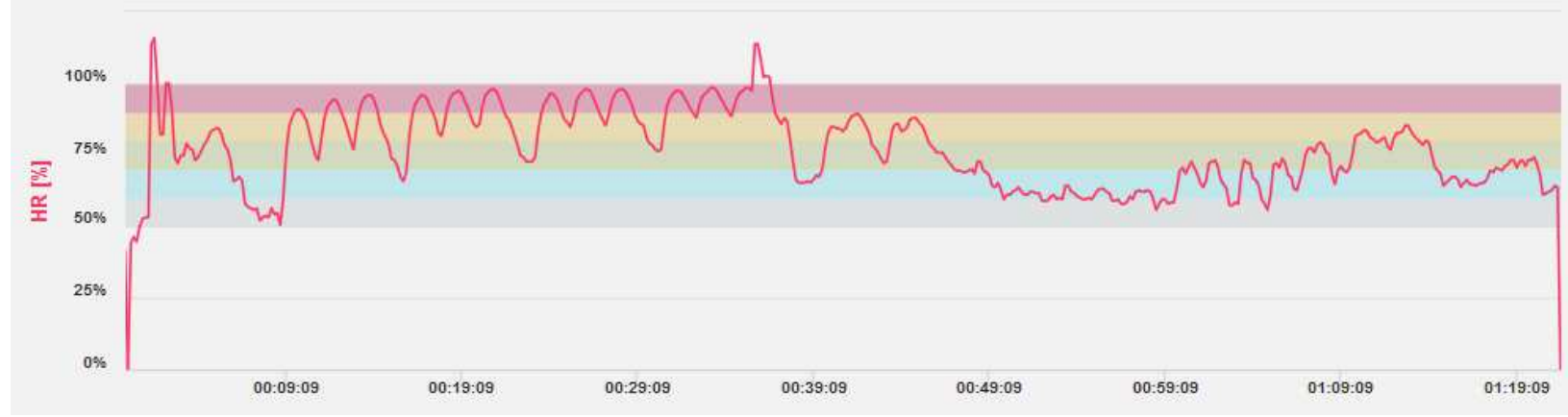

Figure A-11: 2/15/2019.

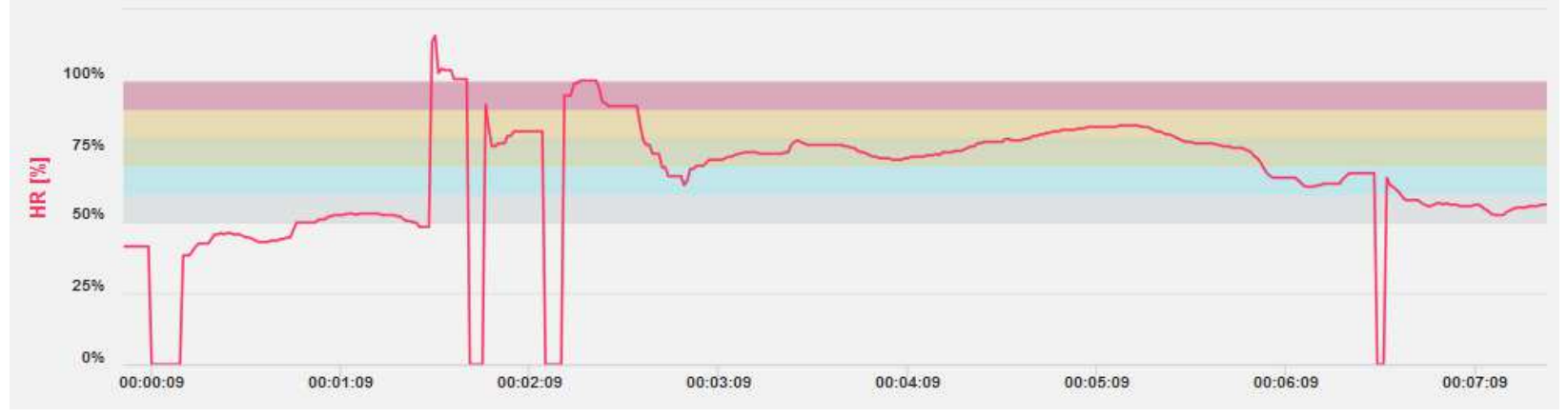

Figure A-12: 2/15/2019, showing close-up of first occurrence from figure 11 (classified as artifact). 


\section{APPENDIX B: RECORDED SESSION IMAGES}

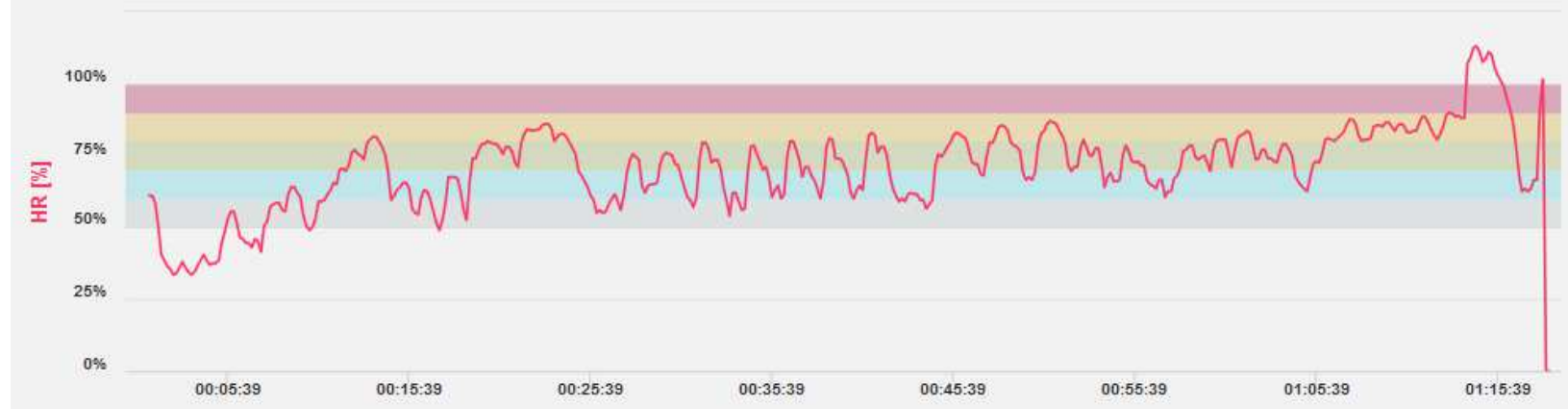

Figure B-1: 6/29/2018, session lasting 01:19:09, 1 occurrence lasting 00:02:58 peak of 226

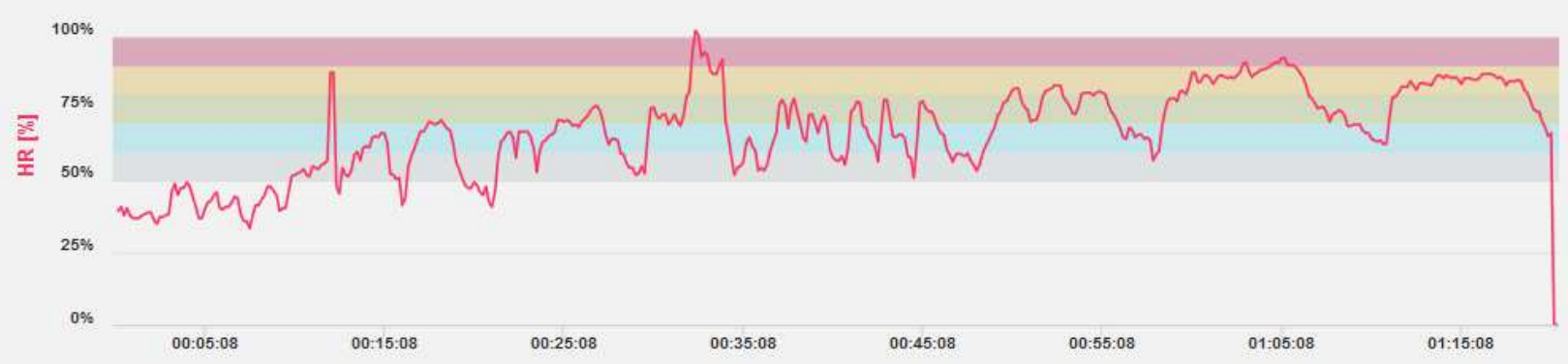

Figure B-2: 7/6/2018, session lasting 01:20:35, 1 occurrence lasting 00:01:46 peak of 204

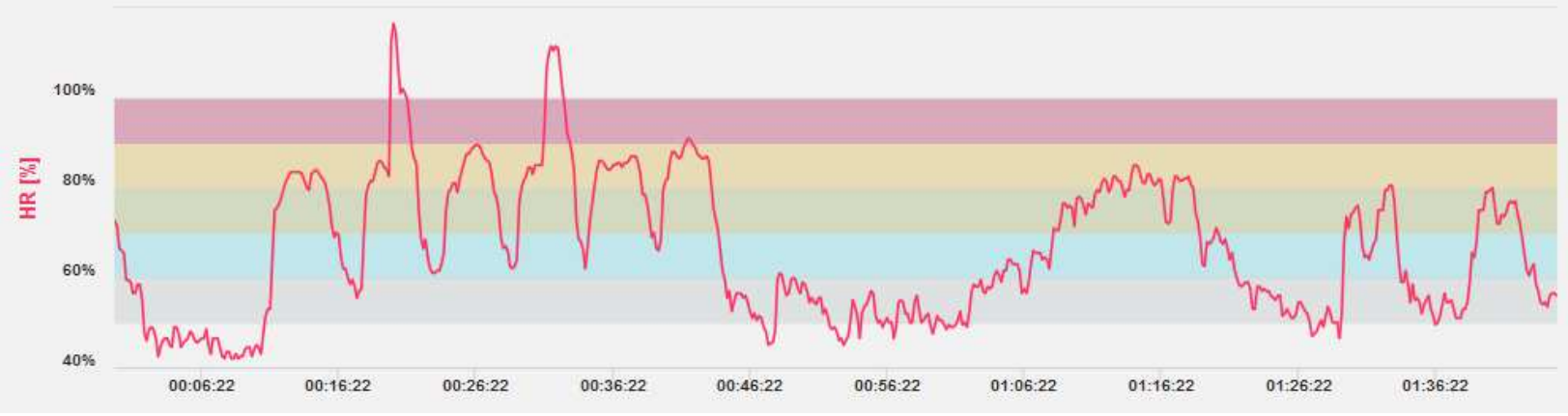

Figure B-3: 7/10/2018, session lasting 01:45:15, 2 occurrences lasting:

00:01:54, with peak of 233

00:02:16, with peak of 223 


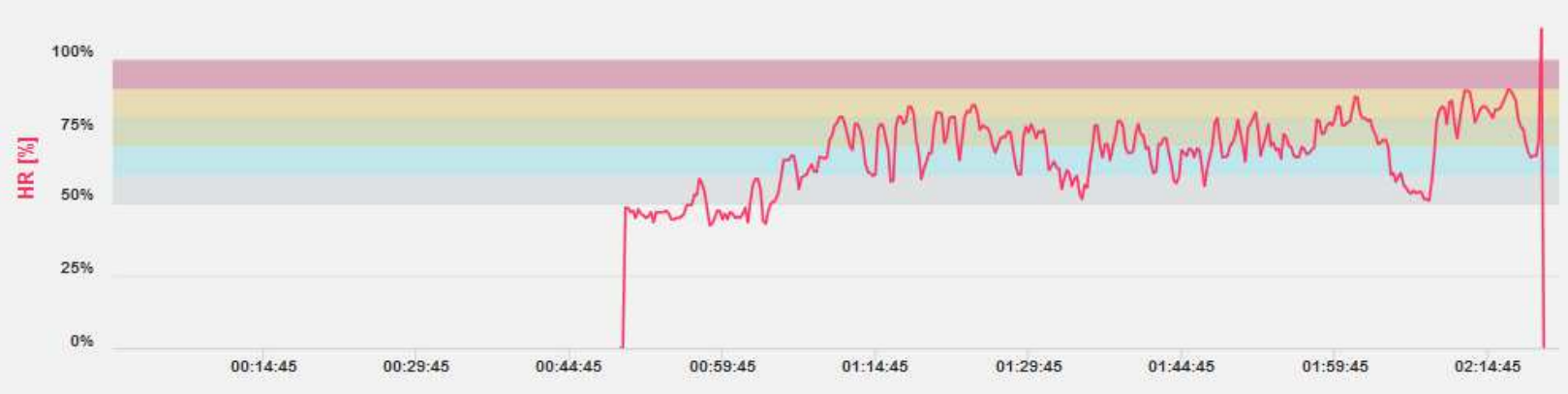

Figure B-4: 7/13/2018, session lasting 02:21:45, 1 occurrence lasting 00:00:06 peak of 221

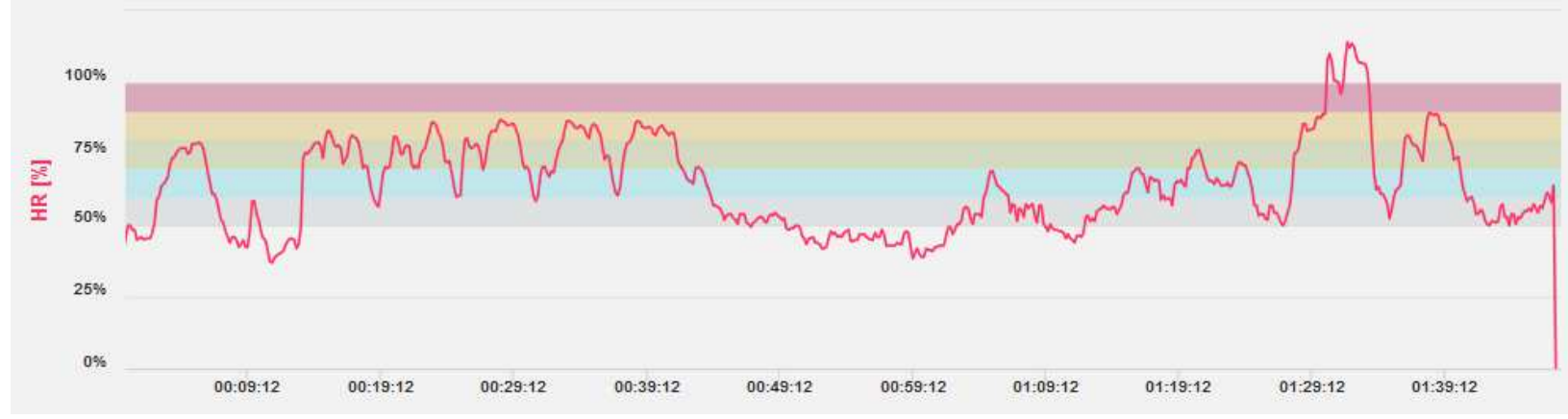

Figure B-5: 7/19/2018, session lasting 01:47:56, 1 occurrence lasting 00:03:40 peak of 228

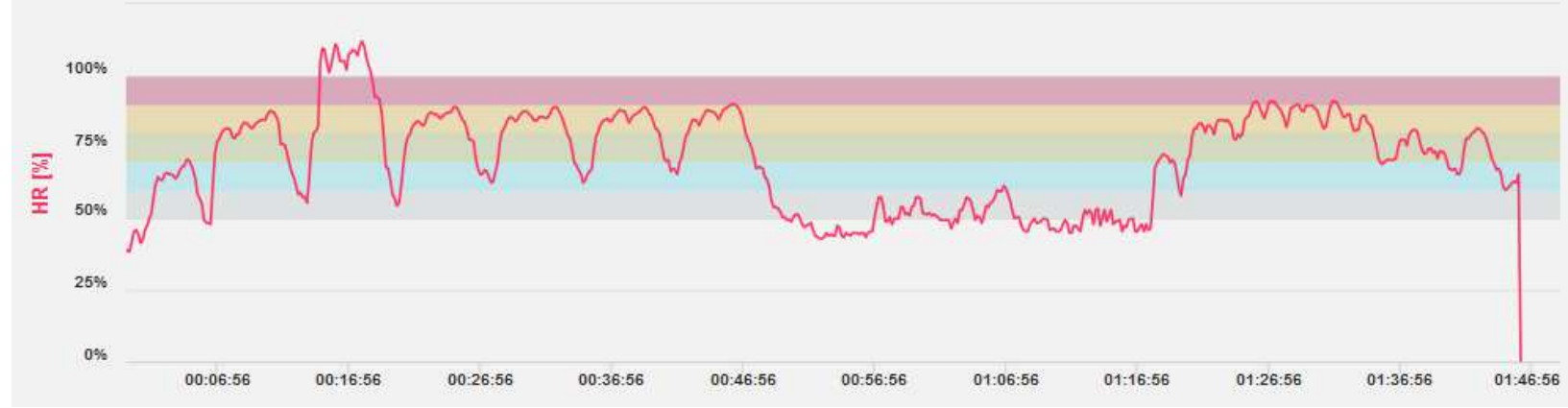

Figure B-6: 7/24/2018, session lasting 01:49:06, 1 occurrence lasting 00:05:01 peak of 224 


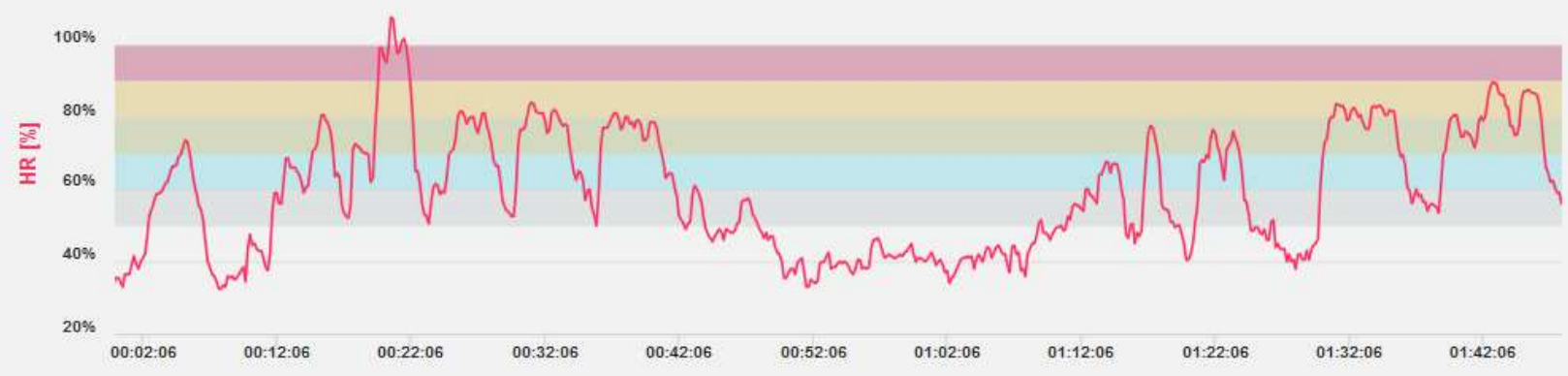

Figure B-7: 7/26/2018, session lasting 01:47:58, 1 occurrence lasting 00:02:57 peak of 215

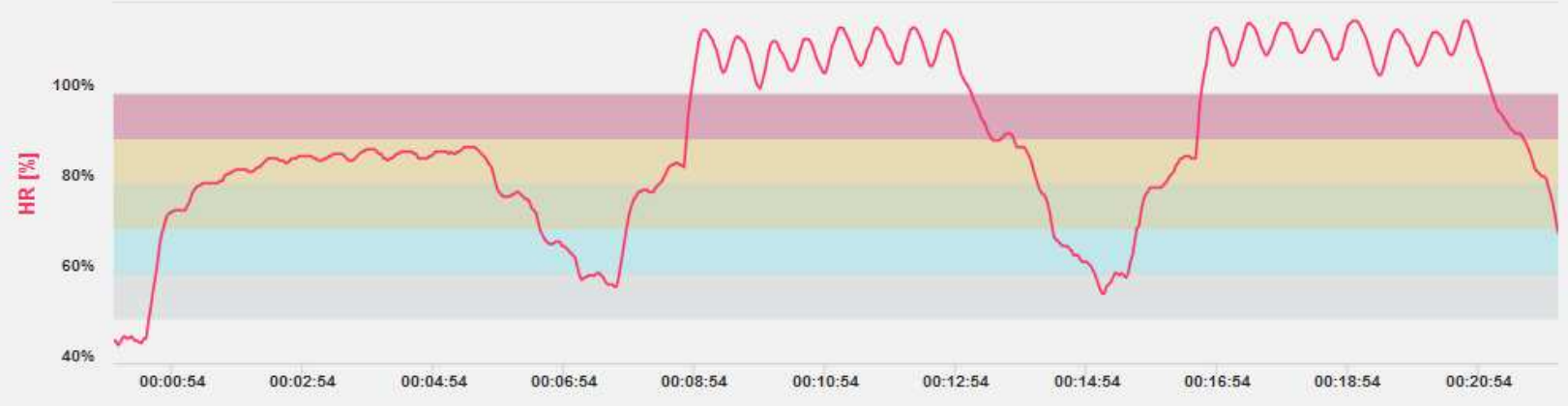

Figure B-8: 7/31/2018, session lasting 00:22:07, 2 occurrences lasting:

00:04:31, with peak of 228

00:05:32, with peak of 232

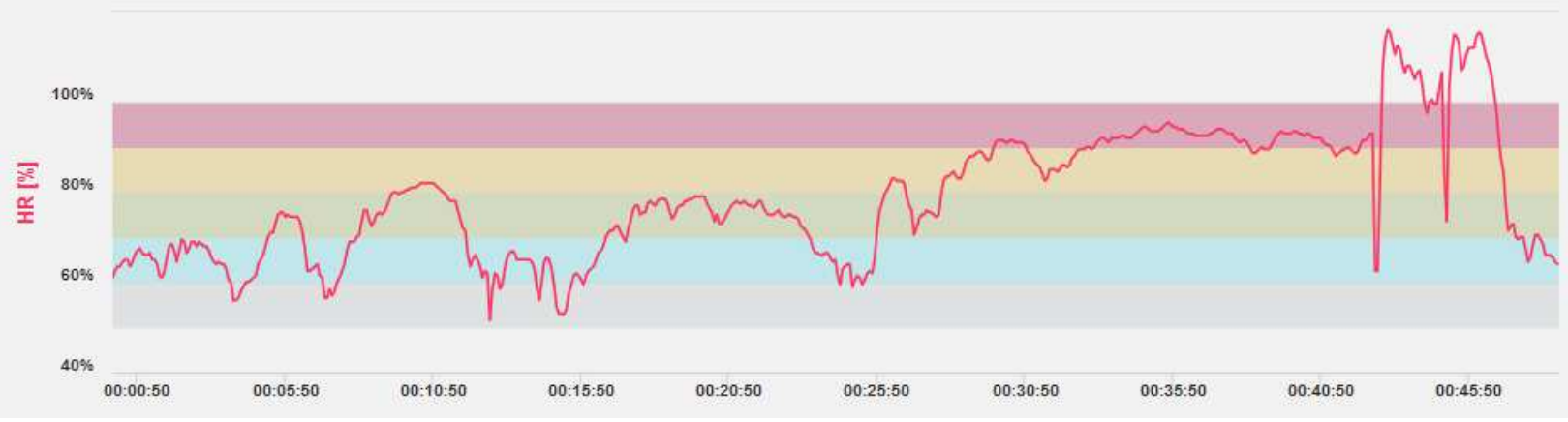

Figure B-9: 7/31/2018, session lasting 00:48:53, 1 occurrence lasting 00:04:15 peak of 232 


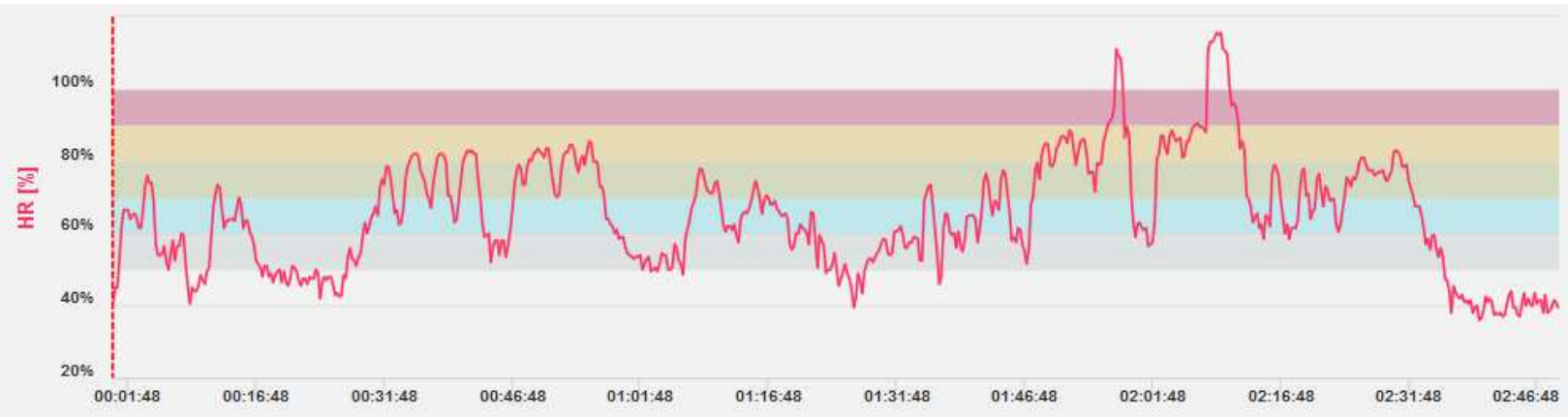

Figure B-10: 8/8/2018, session lasting 02:49:25, 2 occurrences lasting

00:02:00, with peak of 222

00:04:17, with peak of 231

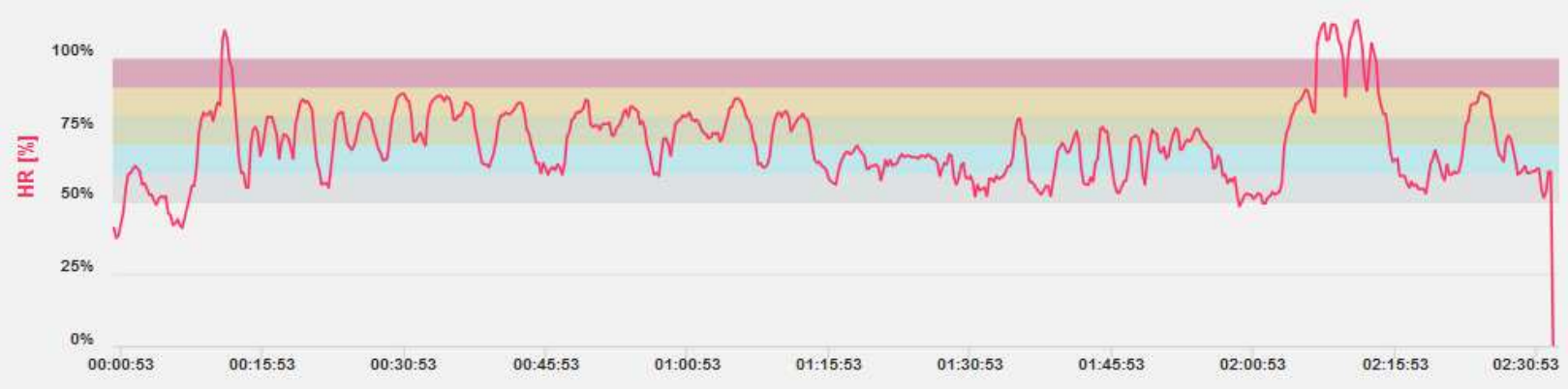

Figure B-11: 8/8/2018, session lasting 02:33:16, 2 occurrences lasting:

00:01:50 with peak of 214

00:07:32 with peak of 226

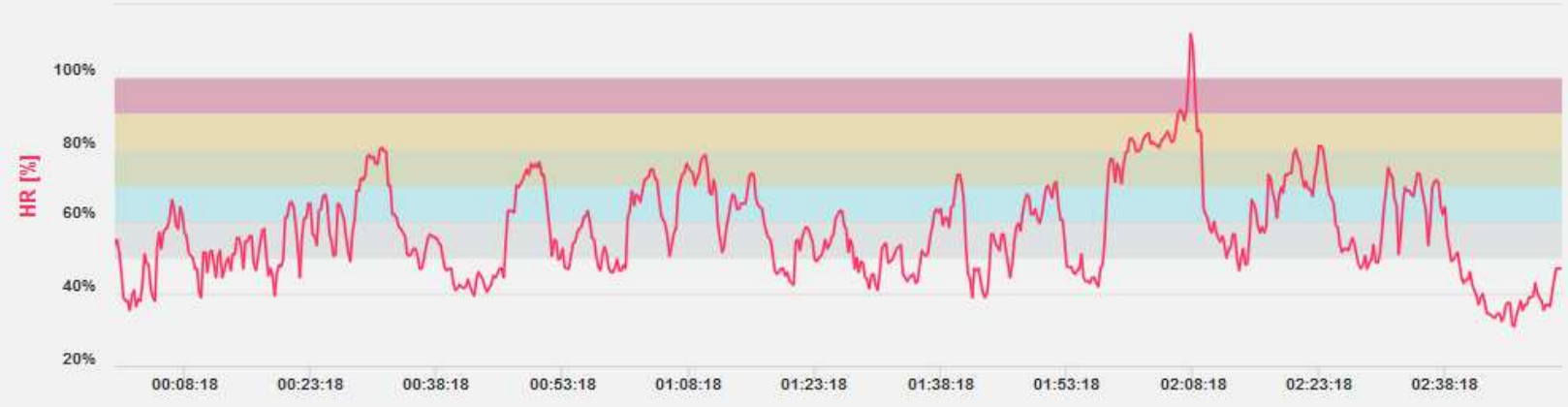

Figure B-12: 8/9/2018, session lasting 02:52:14, 1 occurrence lasting 00:01:39 peak of 224 


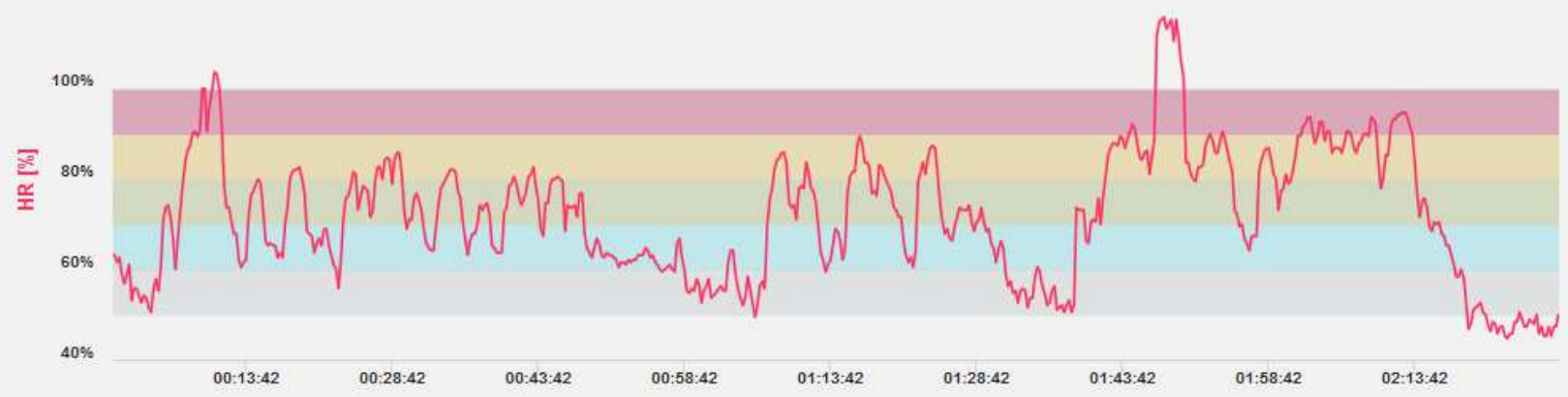

Figure B-13: 8/14/2018, session lasting 00:02:28:31, 2 occurrences lasting:

00:02:14 with peak of 220

00:03:16 with peak of 219

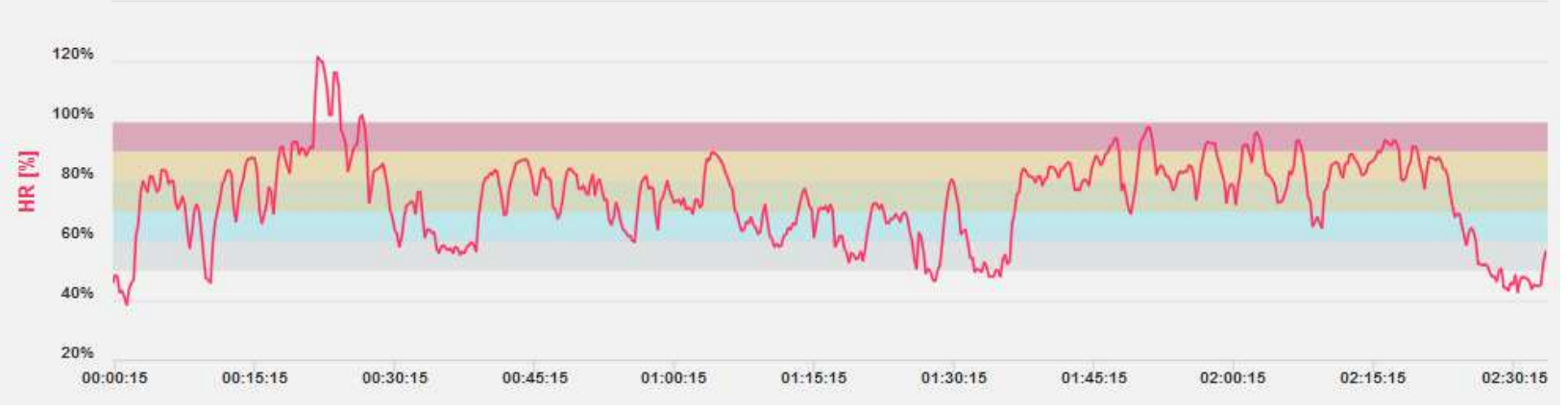

Figure B-14: 8/16/2018, session lasting 02:33:58, 1 occurrence lasting 00:03:16, peak of 231

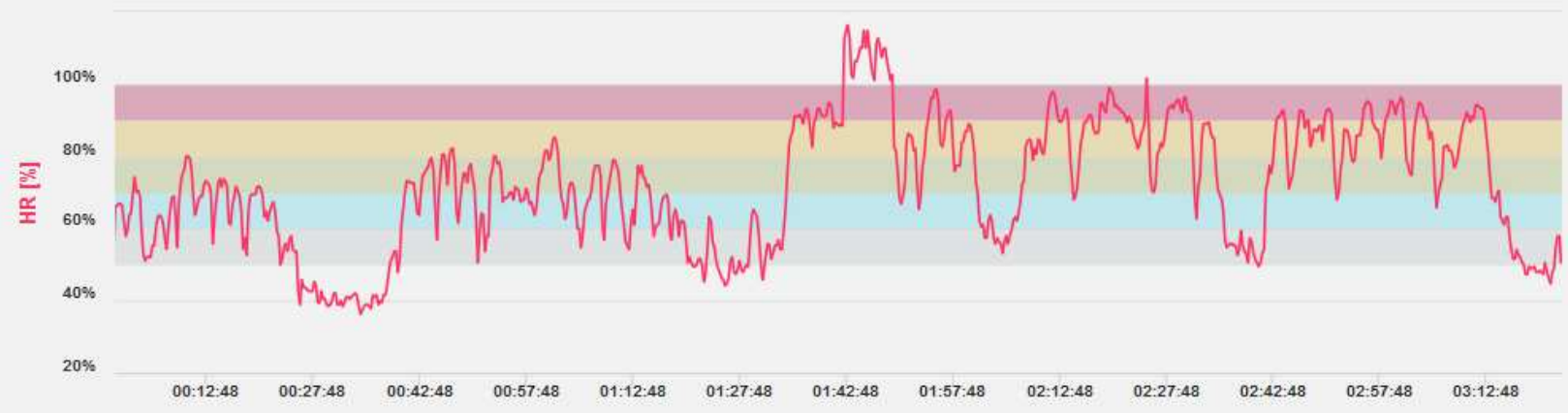

Figure B-15: 8/17/2018, session lasting 03:23:26, 1 occurrence lasting 00:06:58 peak of 221 


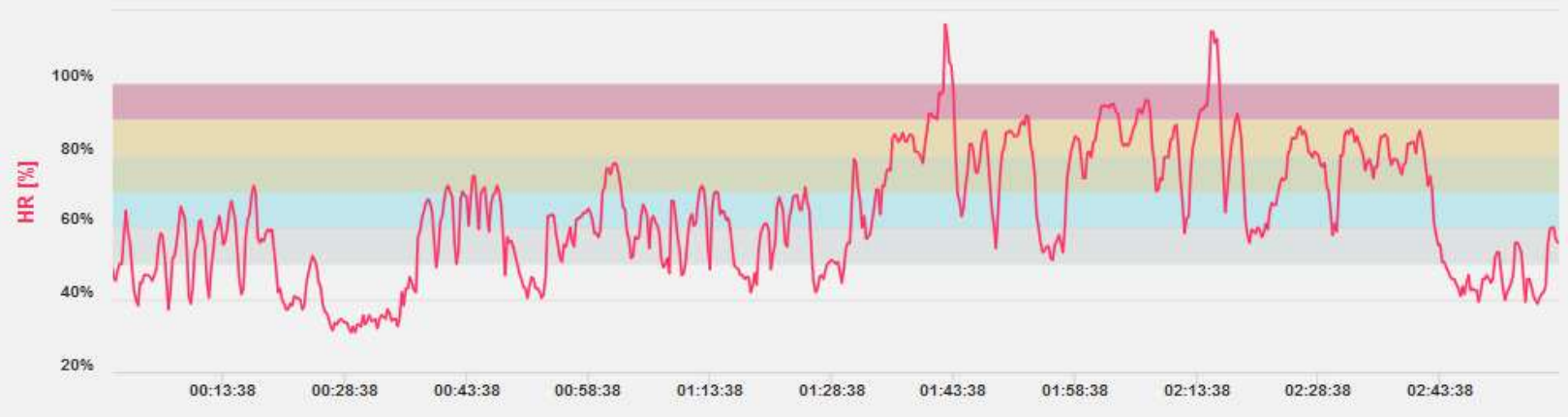

Figure B-16: 8/18/2018, session lasting 02:58:18, 2 occurrences lasting:

00:01:27 with peak of 221

00:02:42 with peak of 217

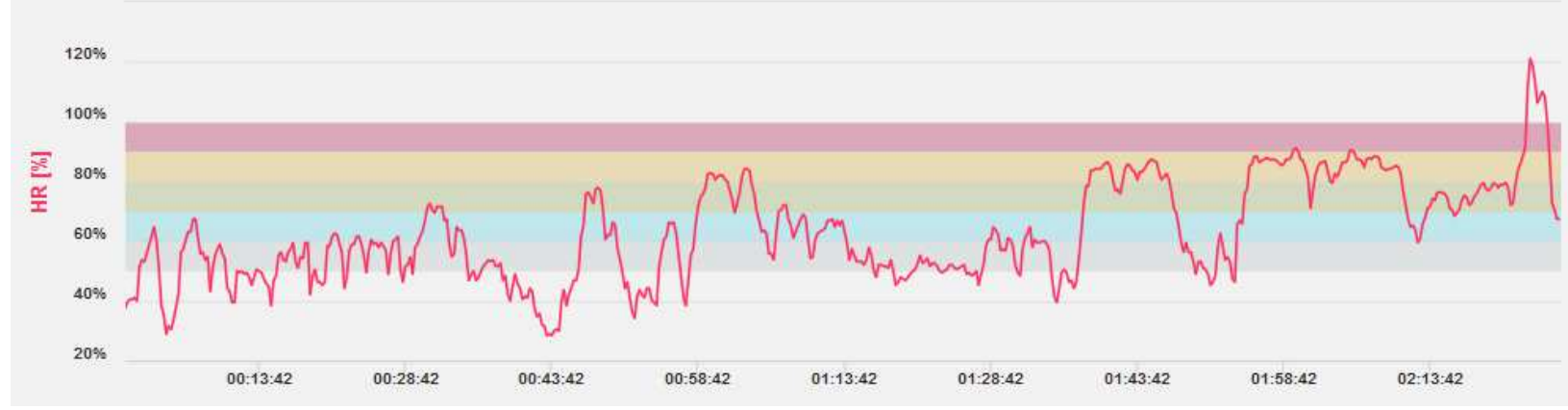

Figure B-17: 8/20/2018, session lasting 02:27:08, 1 occurrence lasting 00:02:42, peak of 230

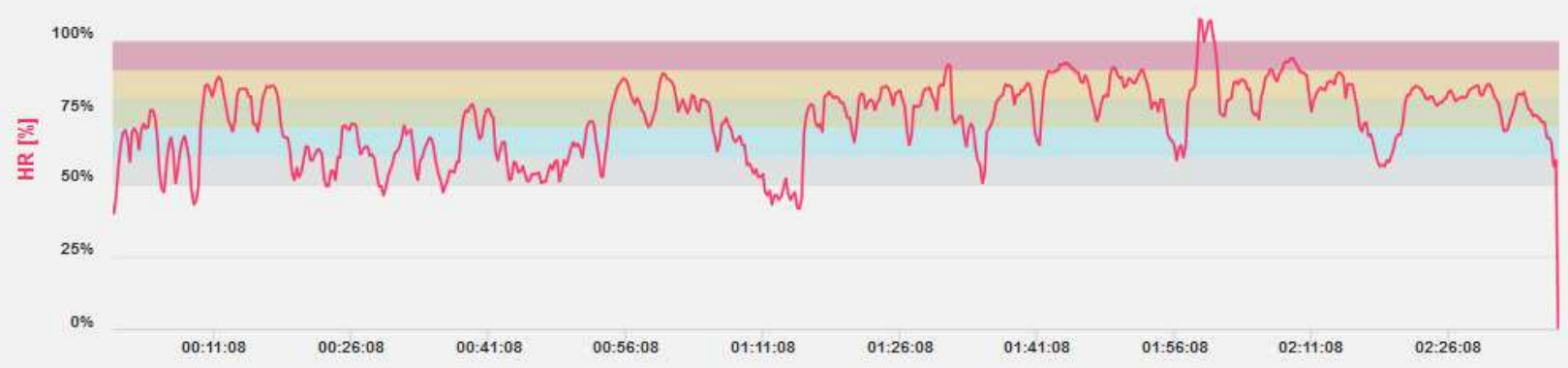

Figure B-18: 8/21/2018, session lasting 02:38:14, 1 occurrence lasting 00:02:19 peak of 204 


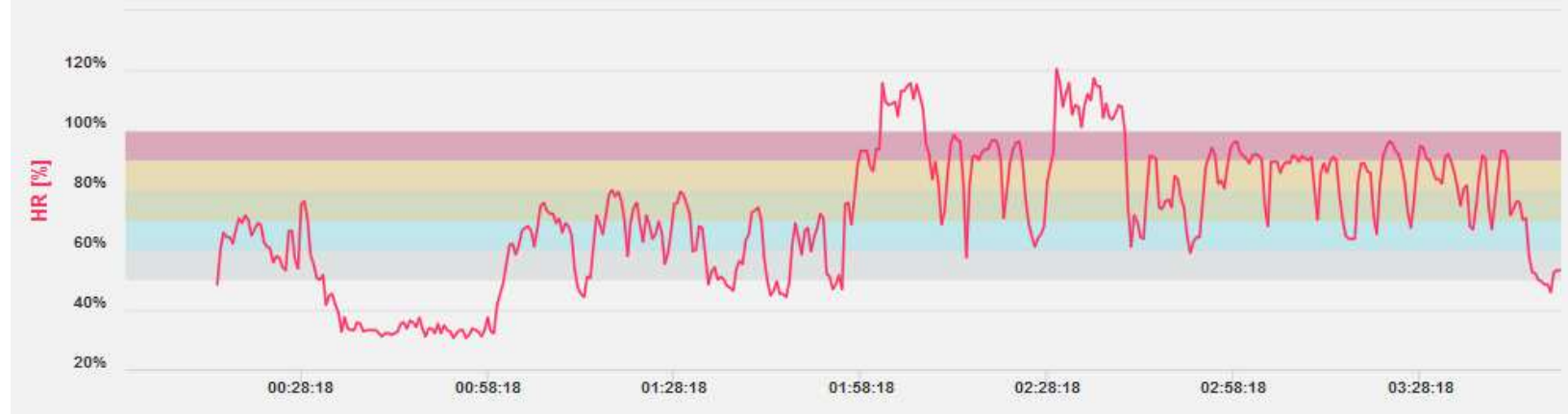

Figure B-19: 8/24/2018, session lasting 03:50:58, 2 occurrences lasting:

00:07:50, with peak of 220

00:12:06, with peak of 229

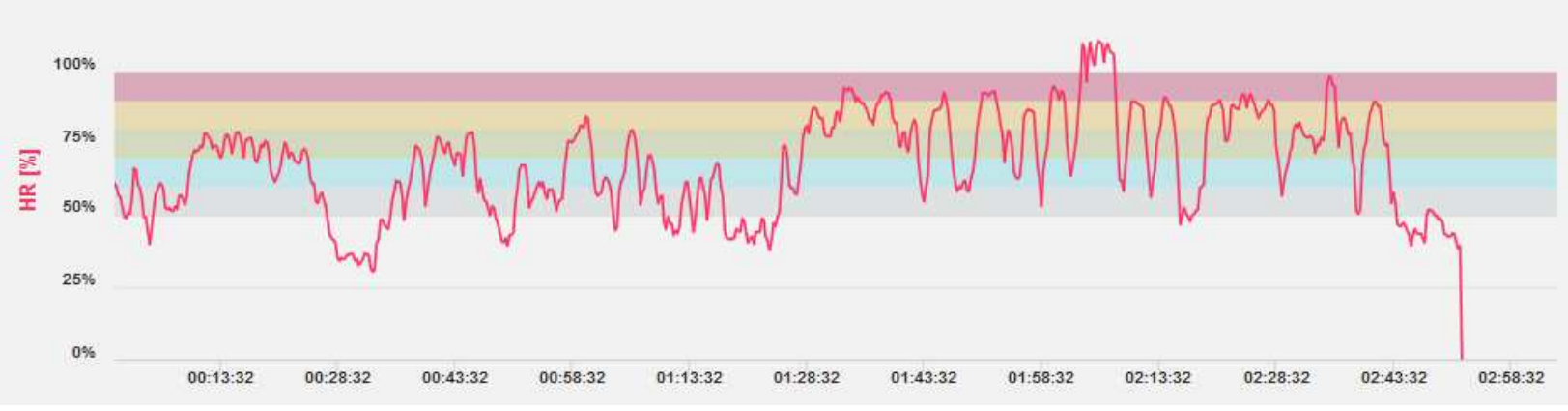

Figure B-20: 8/25/2018, session lasting 03:04:28, 1 occurrence lasting 00:04:56, peak of 210

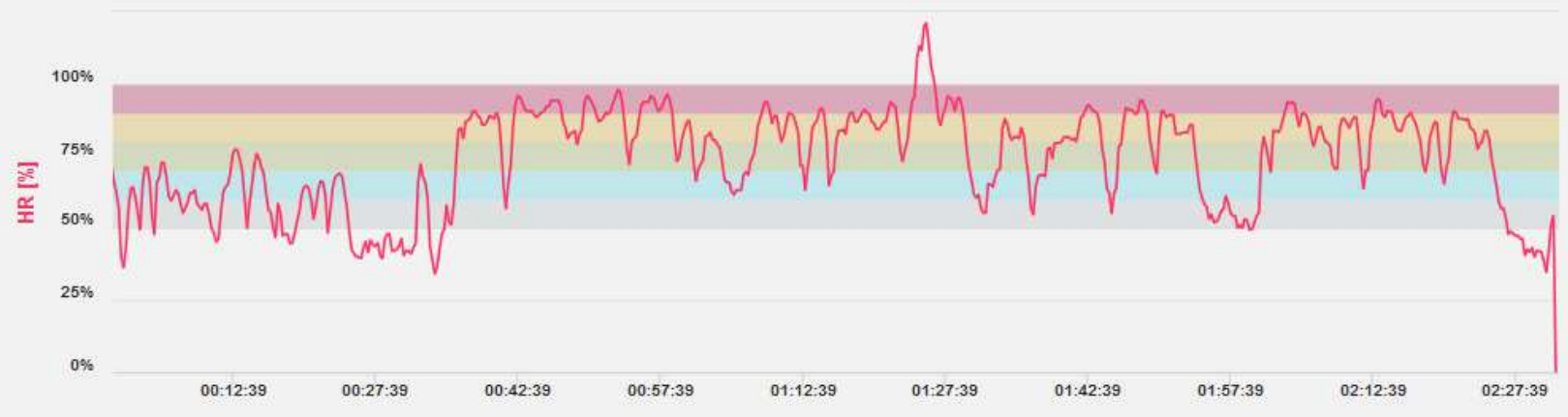

Figure B-21: 8/25/2018, session lasting 02:32:15, 1 occurrence lasting 00:02:14, peak of 230 


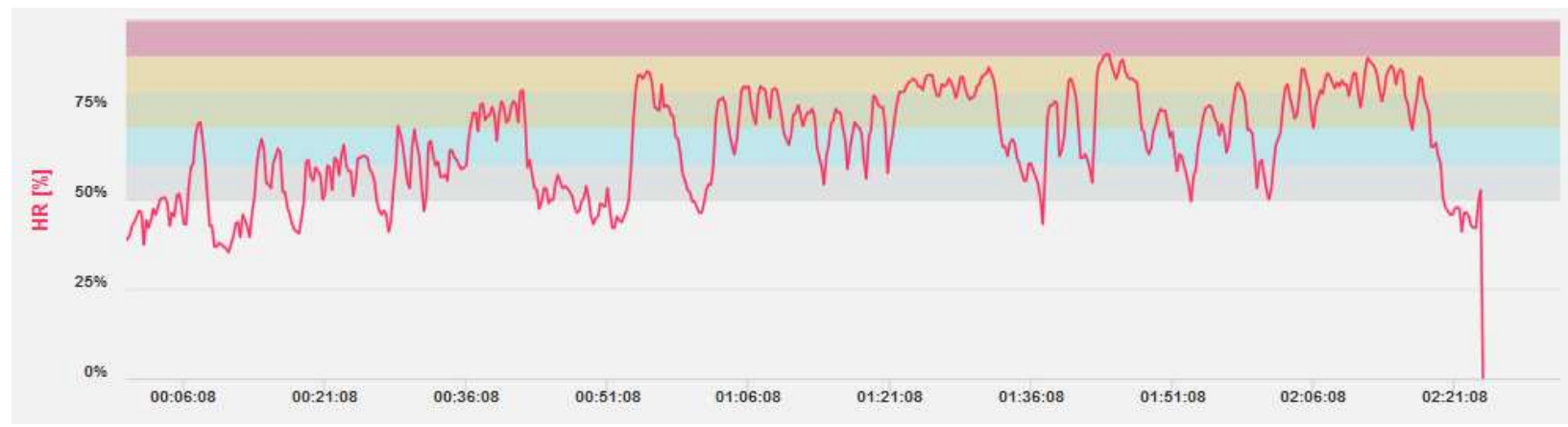

Figure B-22: 8/29/2018, session lasting 02:32:20, 1 occurrence lasting 00:00:14, peak of 156 (sub-maximal)

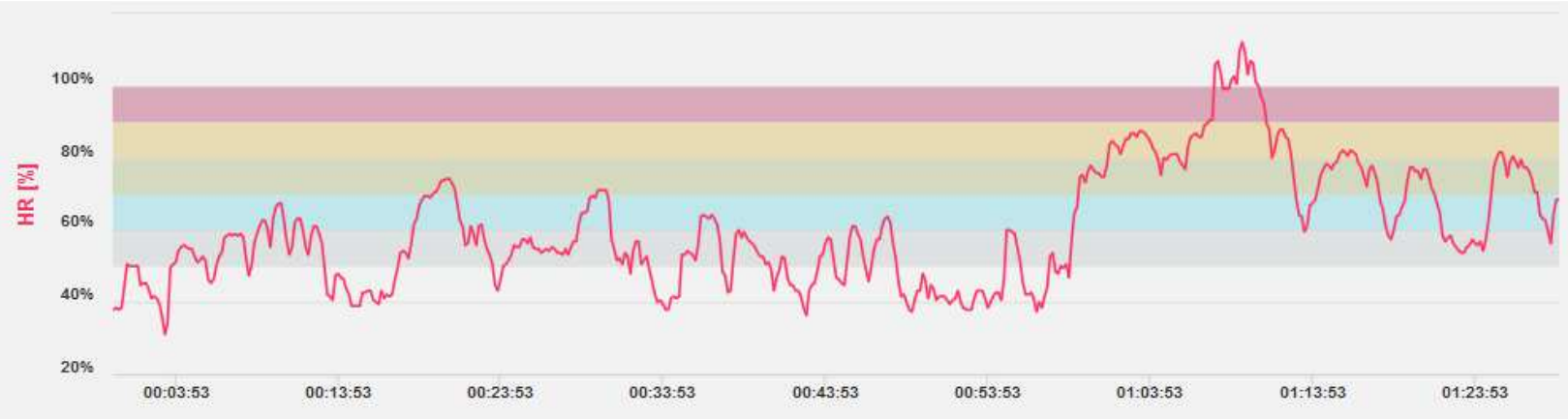

Figure B-23: 9/1/2018, session lasting 01:29:04, 2 occurrences lasting:

00:03:51, with peak of 171 (sub-maximal)

00:03:29, with peak of 213

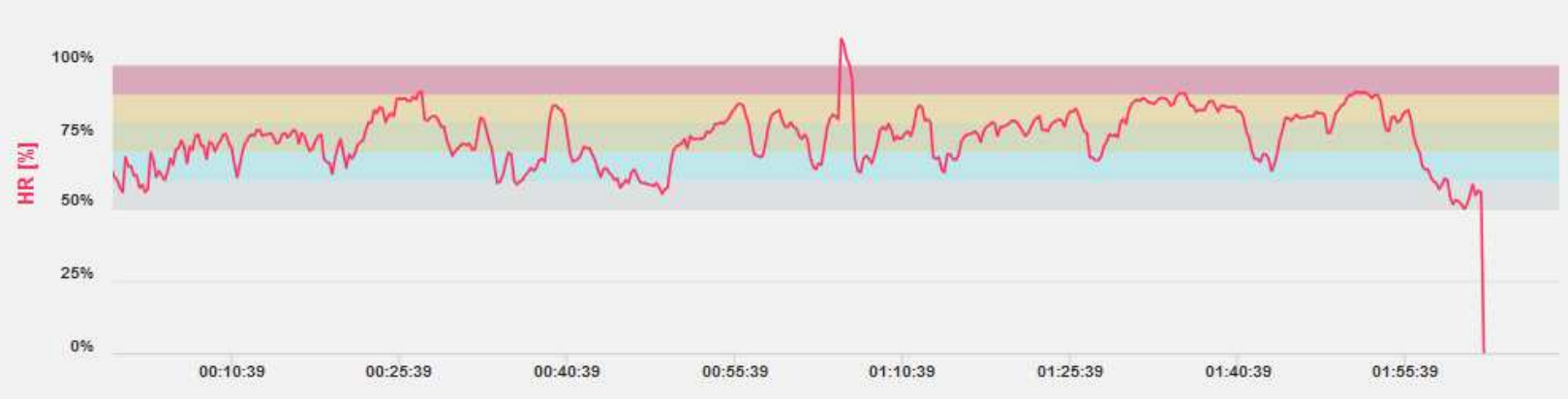

Figure B-24: 9/3/2018, session lasting 02:09:23, 1 occurrence lasting 00:01:28, peak of 207 


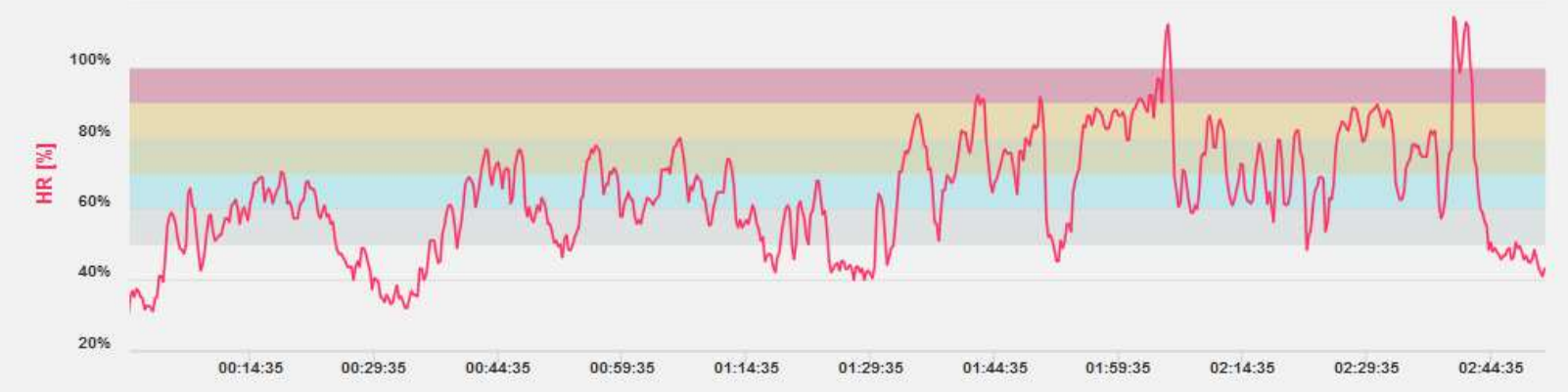

Figure B-25: 9/7/2018, session lasting 02:51:13, 3 occurrences lasting:

00:00:43, with peak of 174 (sub-maximal)

$00: 02: 10$, with peak of 213

00:02:37, with peak of 217

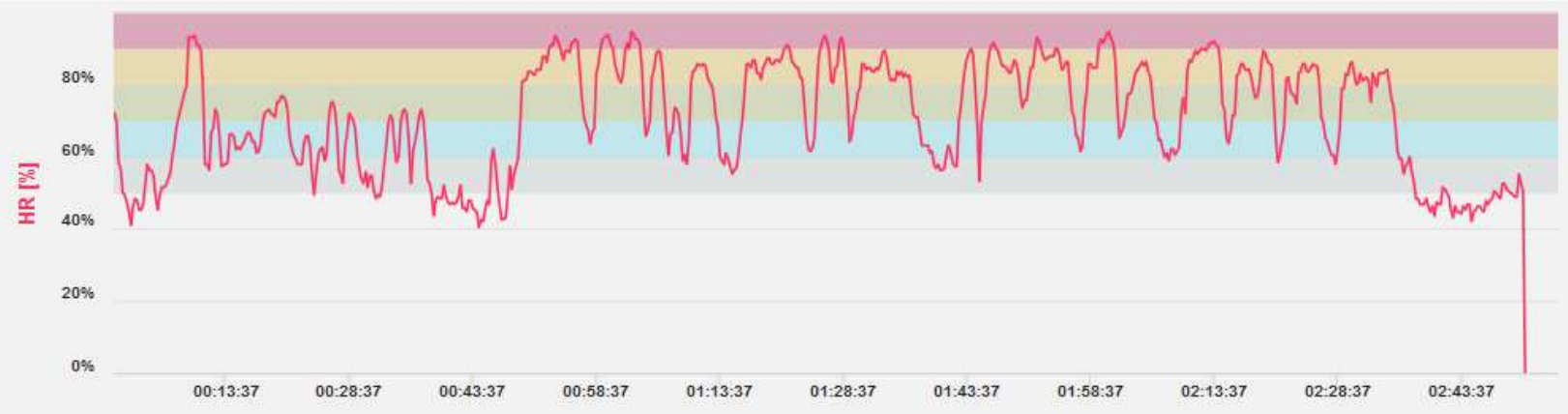

Figure B-26: 9/7/2018, session lasting 02:55:23, 1 occurrence 00:02:06 with peak of 178 (submaximal)

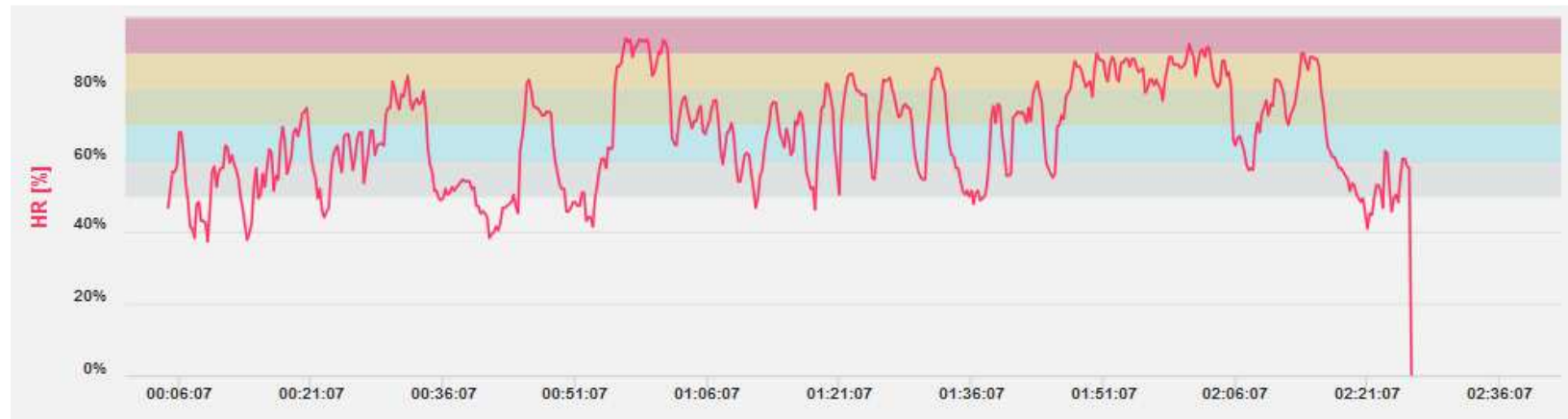

Figure B-27: 9/12/2018, session lasting 02:43:07, 1 occurrence lasting 00:06:28, peak of 179 (sub-maximal) 


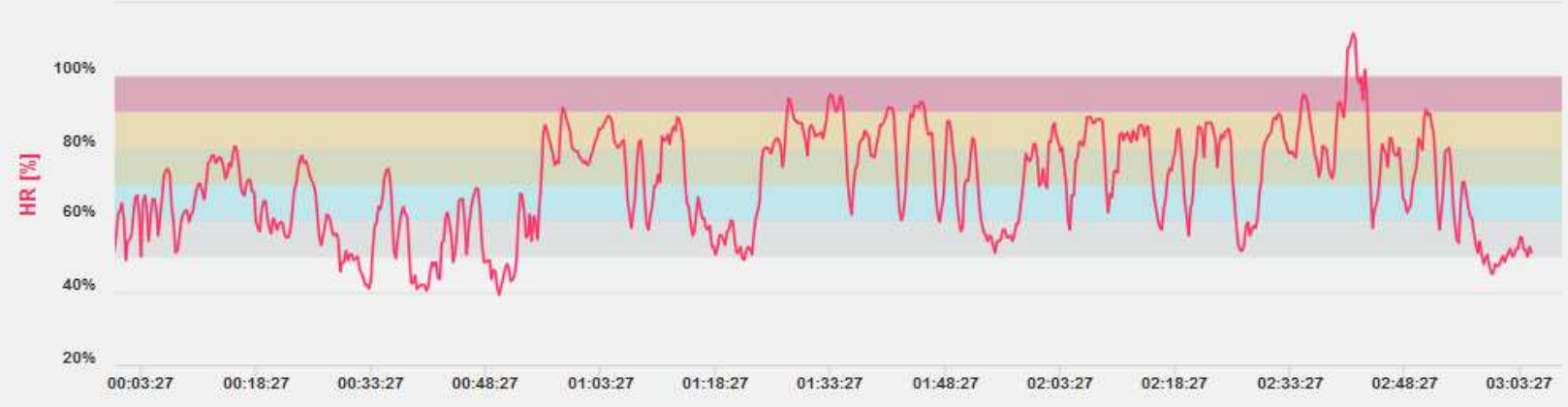

Figure B-28: 9/14/2018, session lasting 03:08:57, 1 occurrence lasting 00:03:05, peak of 212

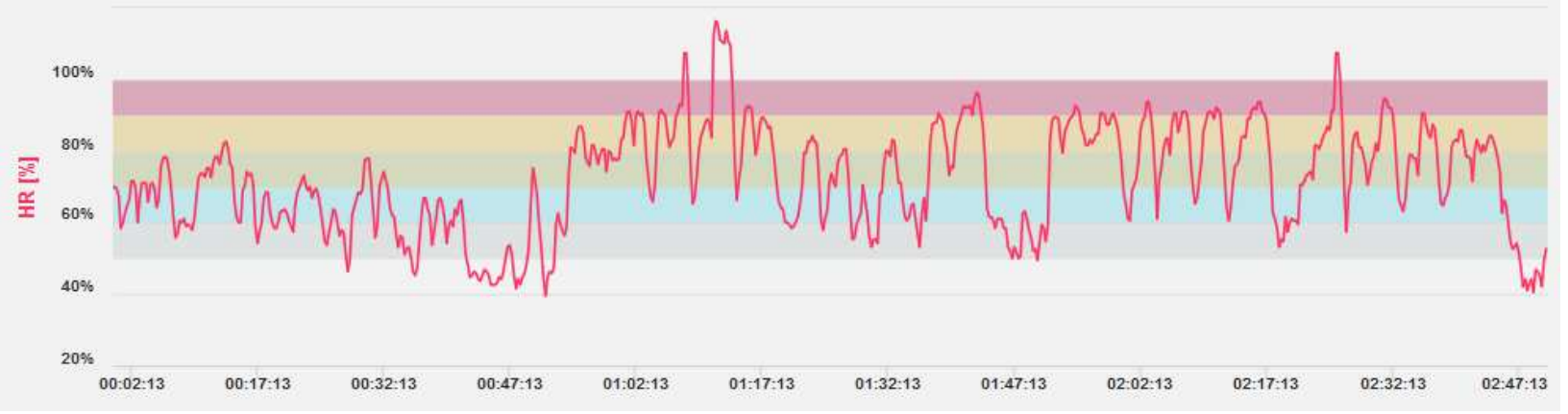

Figure B-29: 9/15/2018, session lasting 02:50:39, 3 occurrences lasting:

00:00:57, with peak of 204

$00: 02: 42$, with peak of 221

00:01:06, with peak of 204

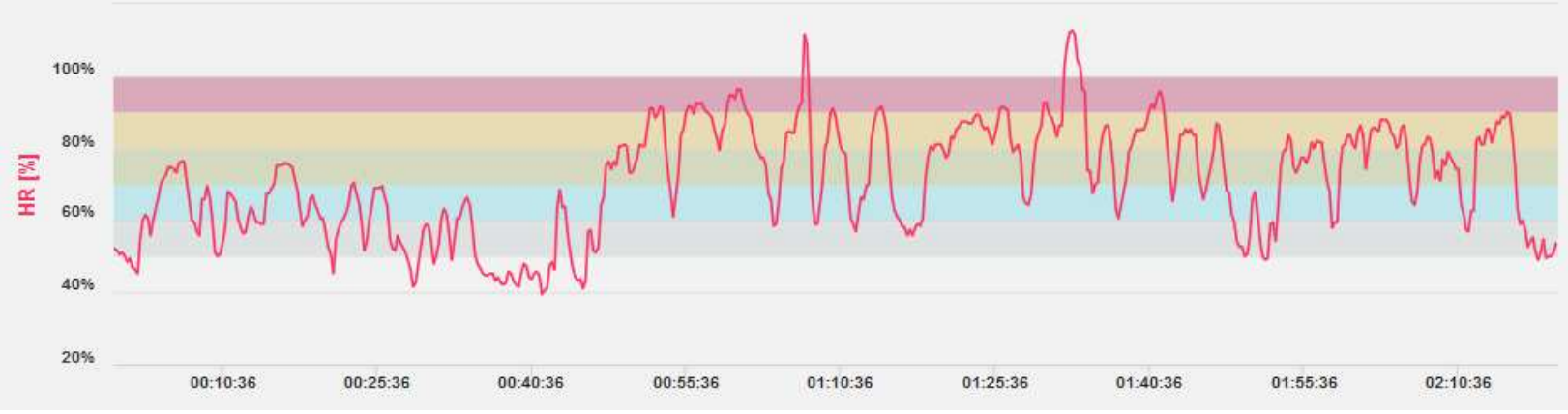

Figure B-30: 9/15/2018, session lasting 02:20:18, 2 occurrences lasting:

00:00:53, with peak of 212 00:02:07, with peak of 214 


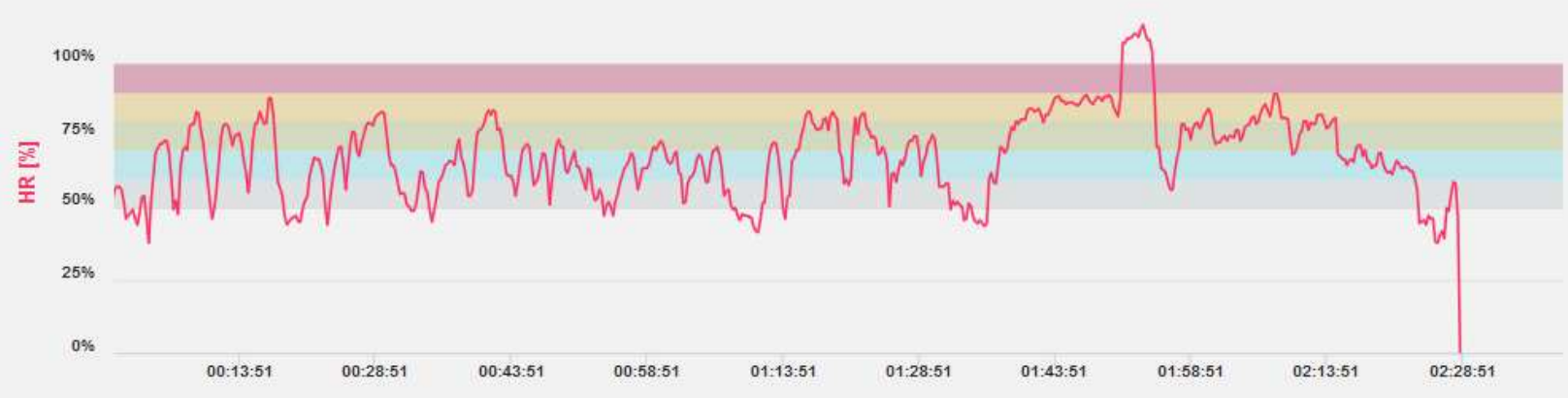

Figure B-31: 9/18/2018, session lasting 02:39:57, 1 occurrence lasting 00:03:47, peak of 215

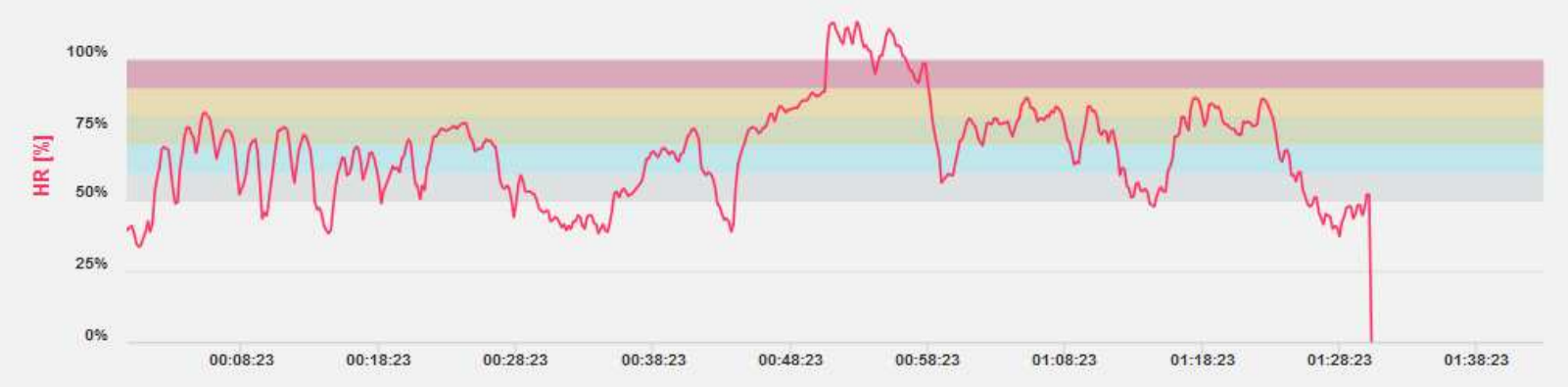

Figure B-32: 9/20/2018, session lasting 01:43:16, 1 occurrence lasting 00:07:20, peak of 215

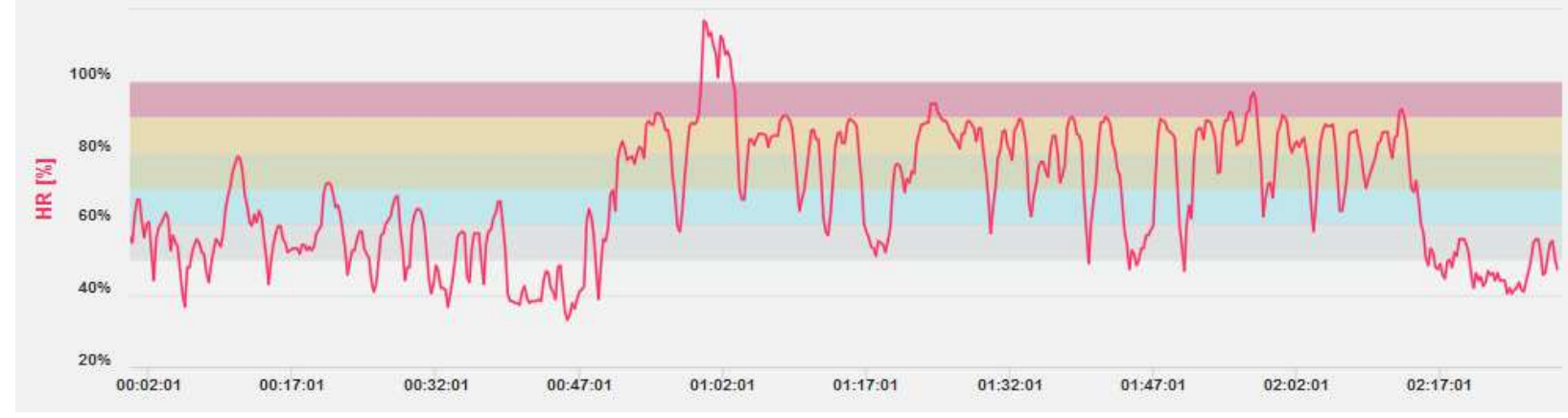

Figure B-33: 9/21/2018, session lasting 02:29:46, 1 occurrence lasting 00:04:23, peak of 222

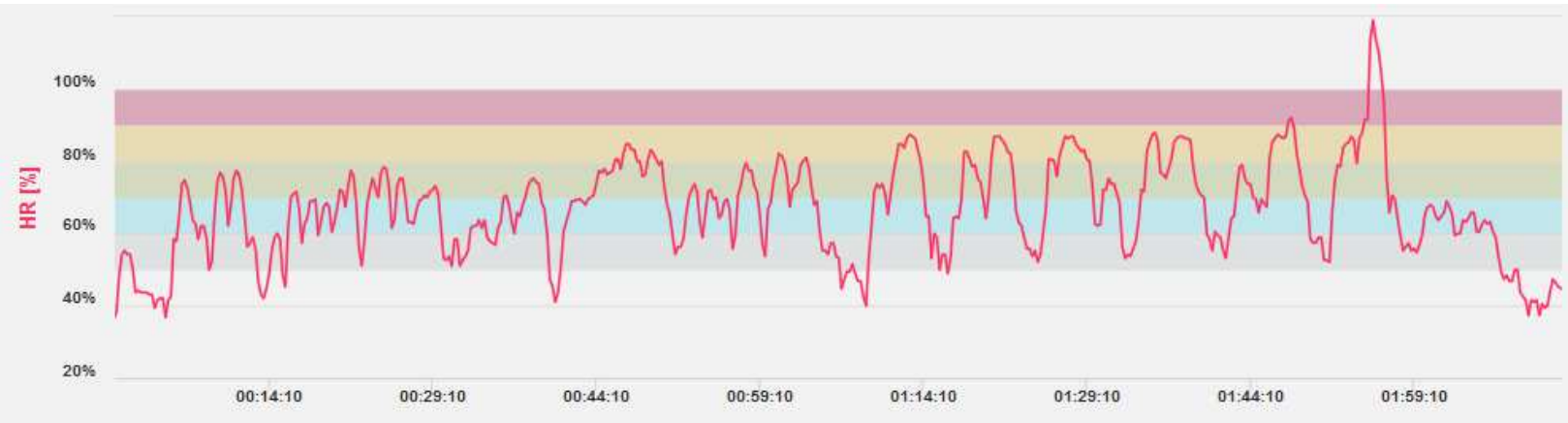

Figure B-34: 9/25/2018, session lasting 02:12:45, 1 occurrence lasting 00:01:50, peak of 226 


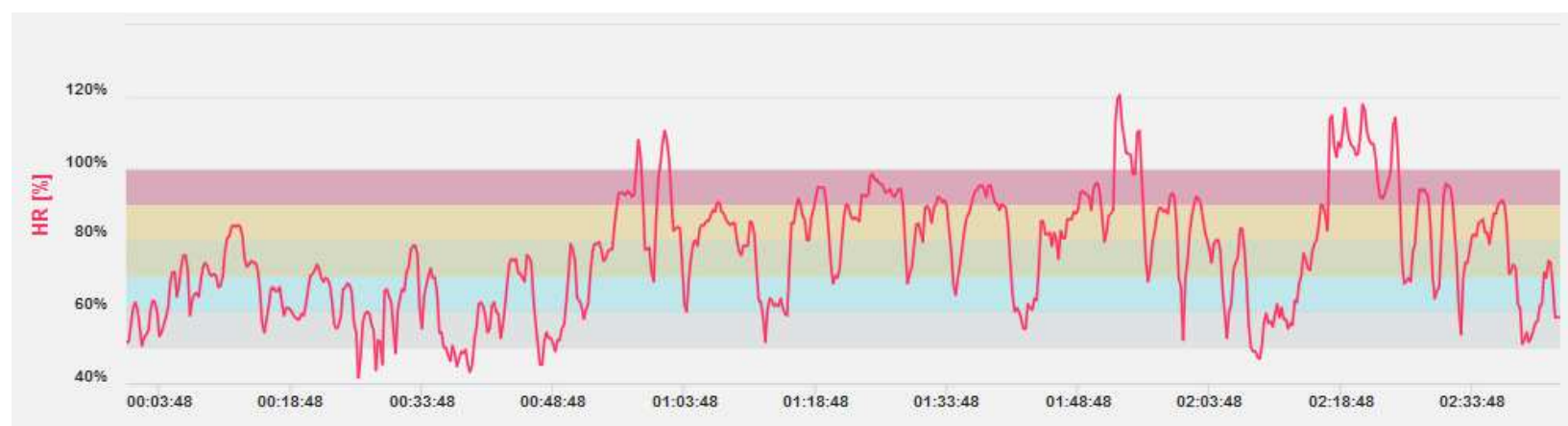

Figure B-35: 9/29/2018, session lasting 02:12:45, 4 occurrences lasting:

00:01:03 with peak of 205

$00: 01: 31$ with peak of 210

00:03:45 with peak of 229

00:08:09 with peak of 224

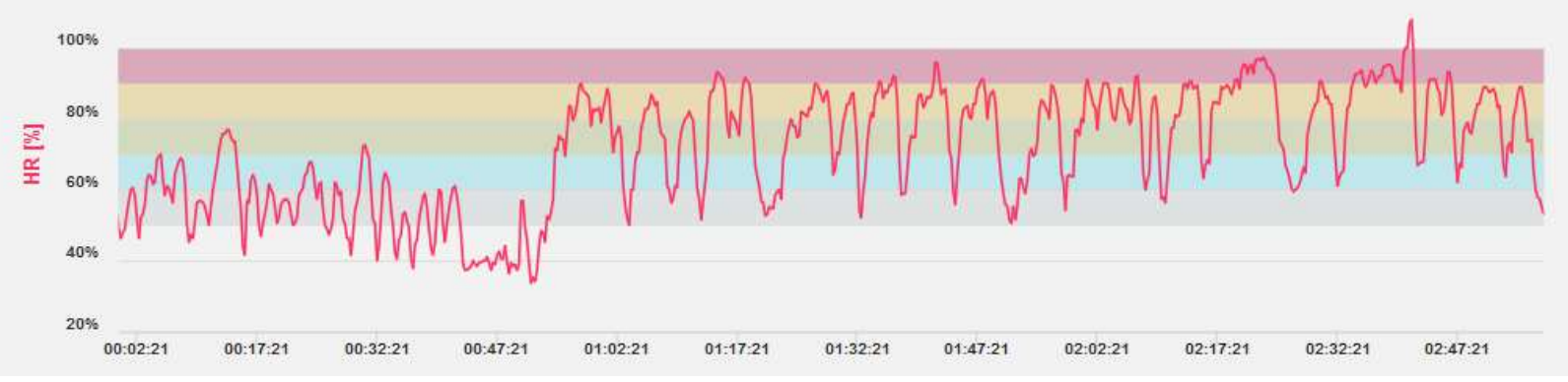

Figure B-36: 10/6/2018, session lasting 02:58:09, 1 occurrence lasting 00:01:48, peak of 205

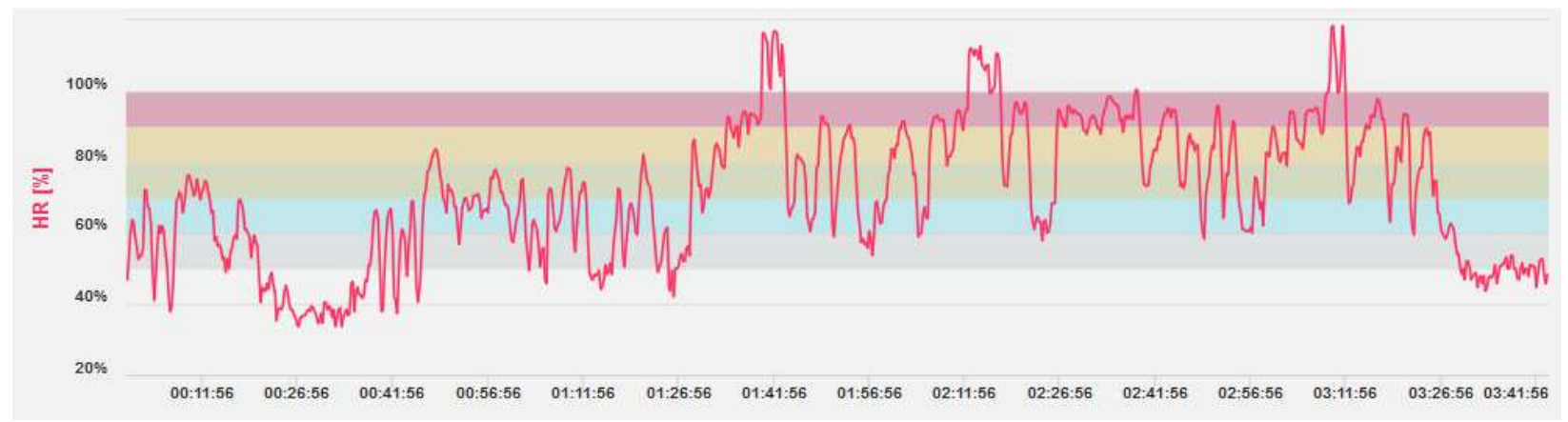

Figure B-37: 10/12/2018, session lasting 03:43:54, 3 occurrences lasting:

00:03:57 with peak of 222

00:05:16 with peak of 214

00:02:55 with peak of 225 


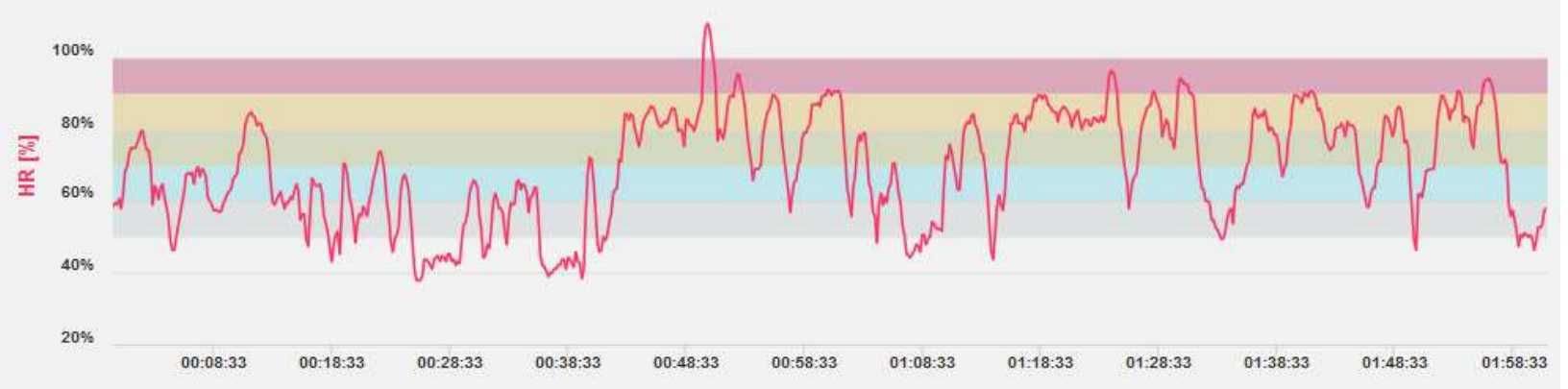

Figure B-38: 10/13/2018, session lasting 02:01:31, 1 occurrence lasting 00:01:16 peak of 208

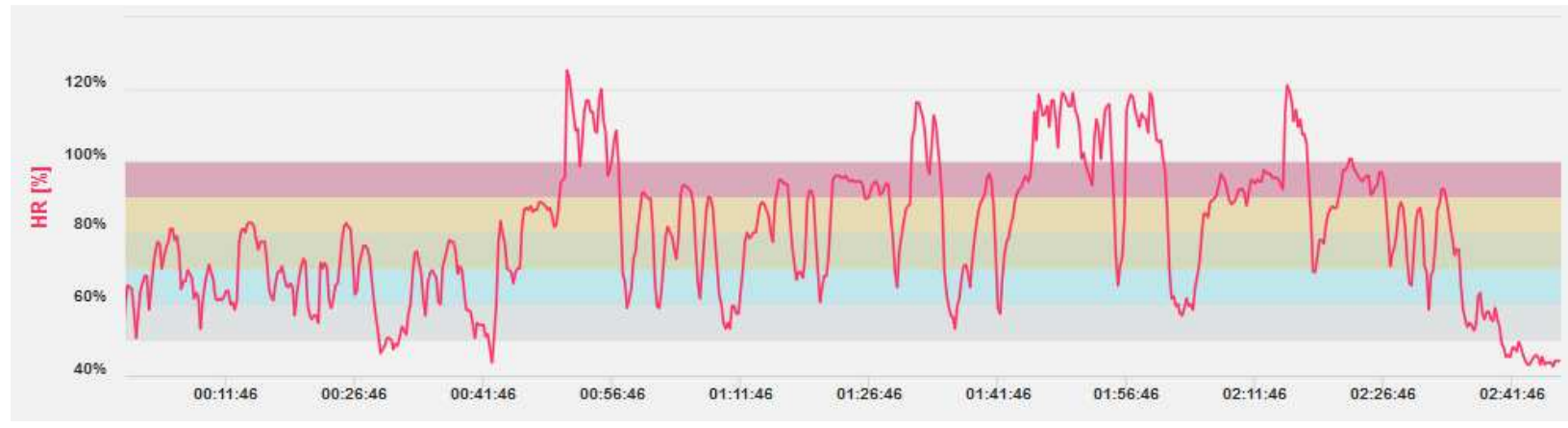

Figure B-39: 10/15/2018, session lasting 02:47:27, 5 occurrences lasting:

00:06:15 with peak of 238

00:03:52 with peak of 221

00:09:41 with peak of 226

00:02:51 with peak of 226

00:03:03 with peak of 230

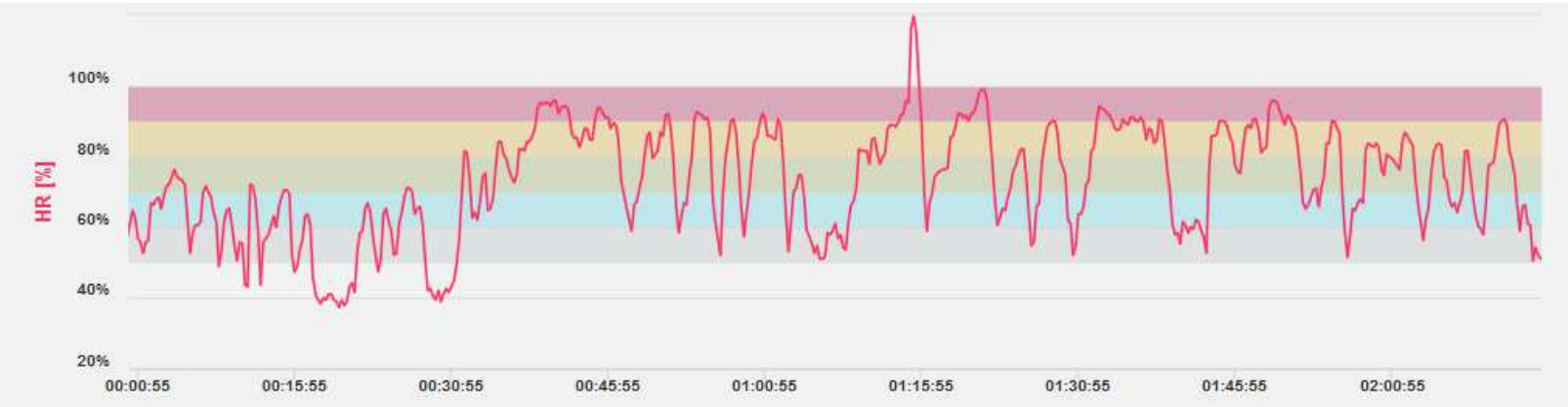

Figure B-40: 10/20/2018, session lasting 02:15:16, 1 occurrence lasting 00:01:30 peak of 228 


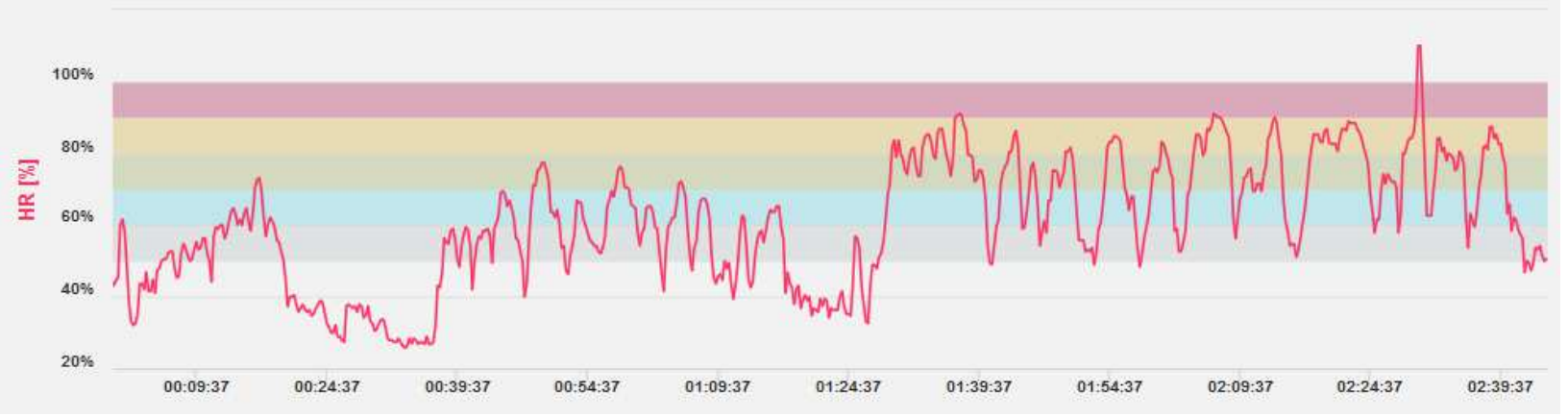

Figure B-41: 10/26/2018, session lasting 02:45:01, 1 occurrence lasting 00:01:01 peak of 209

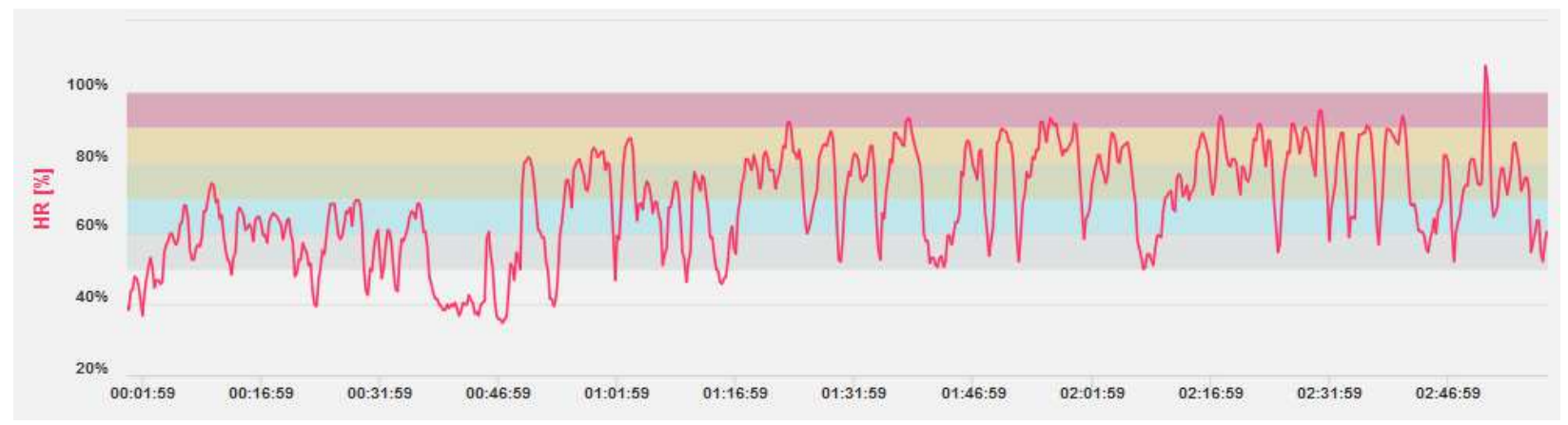

Figure B-42: 10/27/2018, session lasting 02:59:37, 1 occurrence lasting 00:00:57 peak of 204

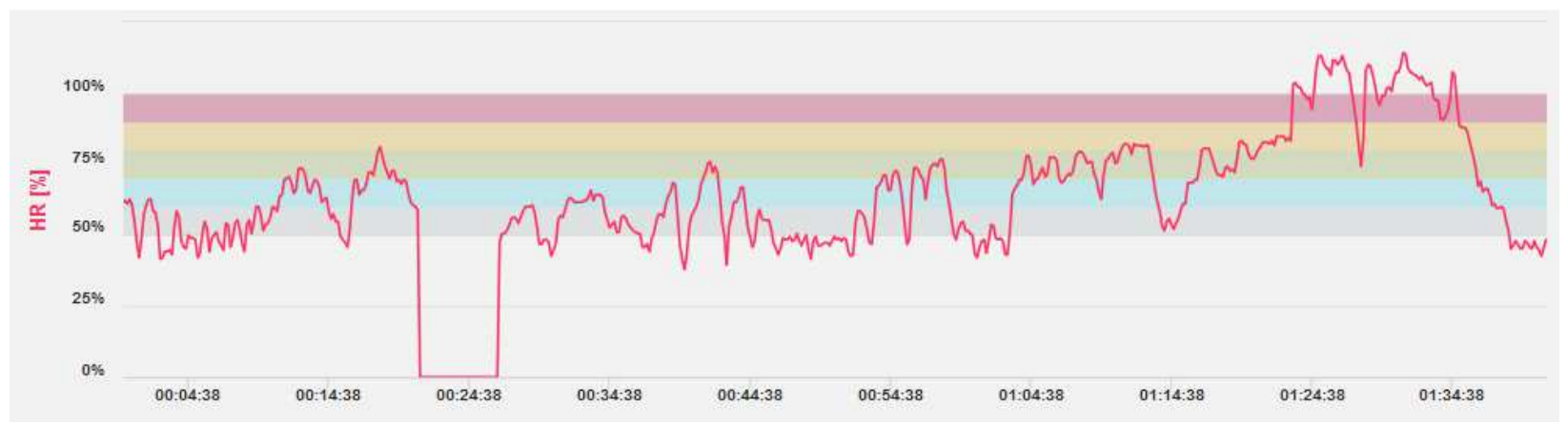

Figure B-43: 11/7/2018, session lasting 01:41:22, 1 occurrence lasting 00:15:33 peak of 217 


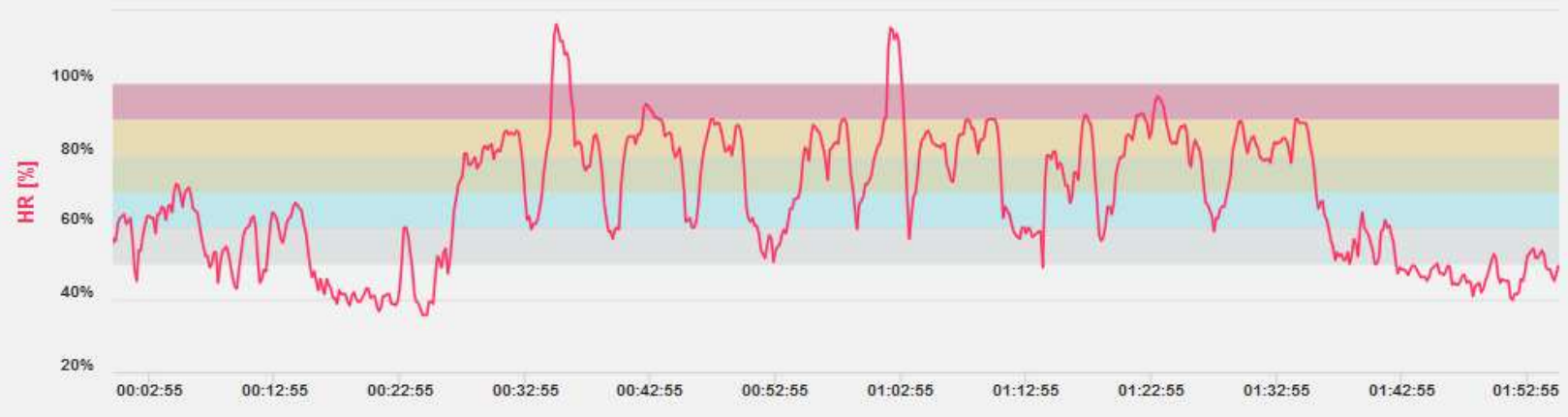

Figure B-44: 11/9/2018, session lasting 01:55:28, 2 occurrences lasting:

00:01:47 with peak of 221

00:01:44 with peak of 219

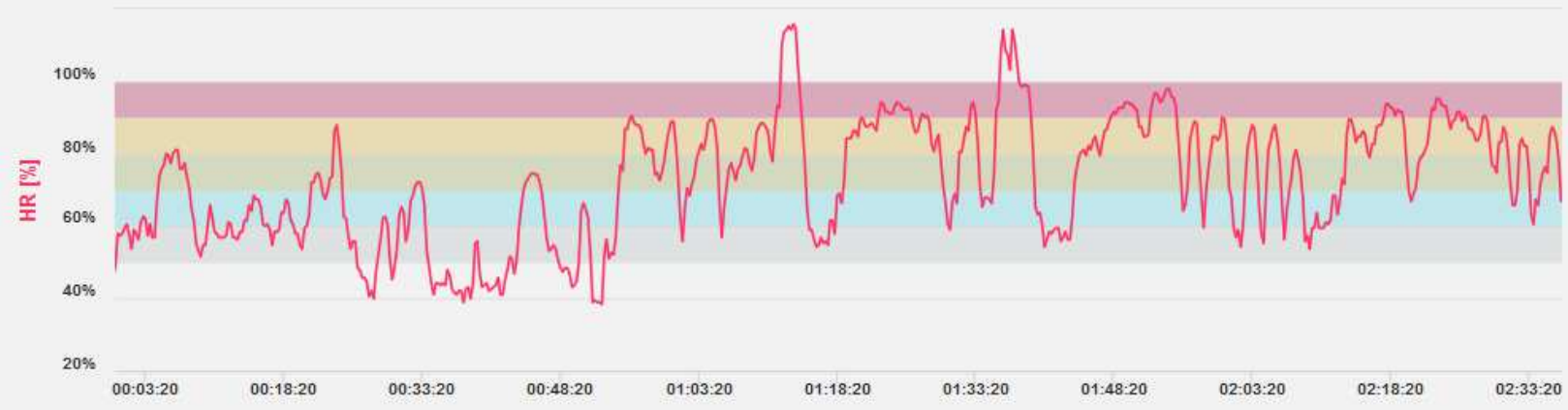

Figure B-45: 11/10/2018, session lasting 02:36:56, 2 occurrences lasting:

00:03:40 with peak of 220

00:04:29 with peak of 217

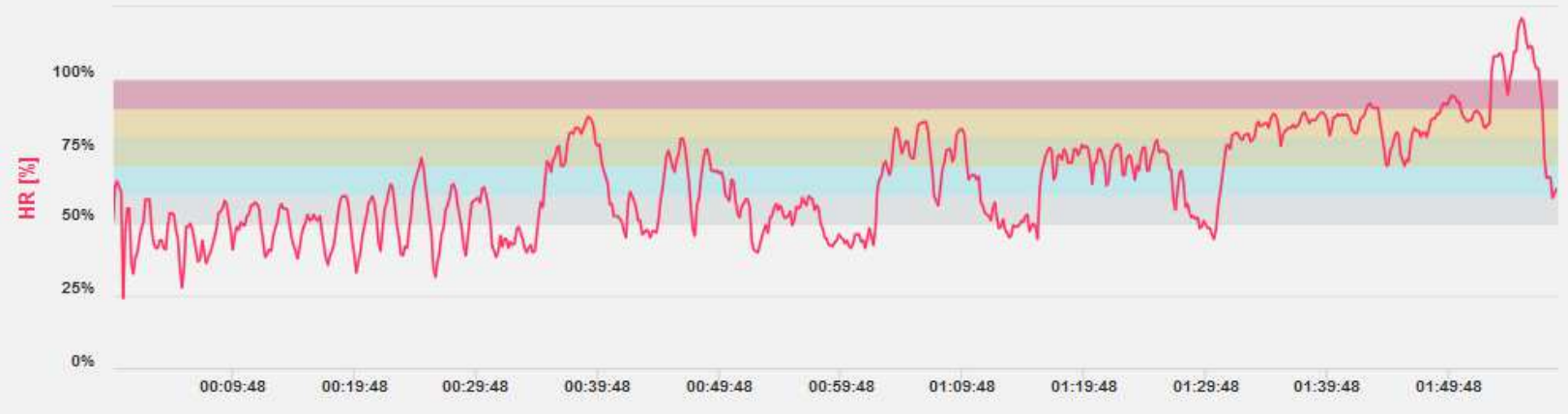

Figure B-46: 11/13/2018, session lasting 01:58:46, 1 occurrence lasting00:04:29 peak of 230 


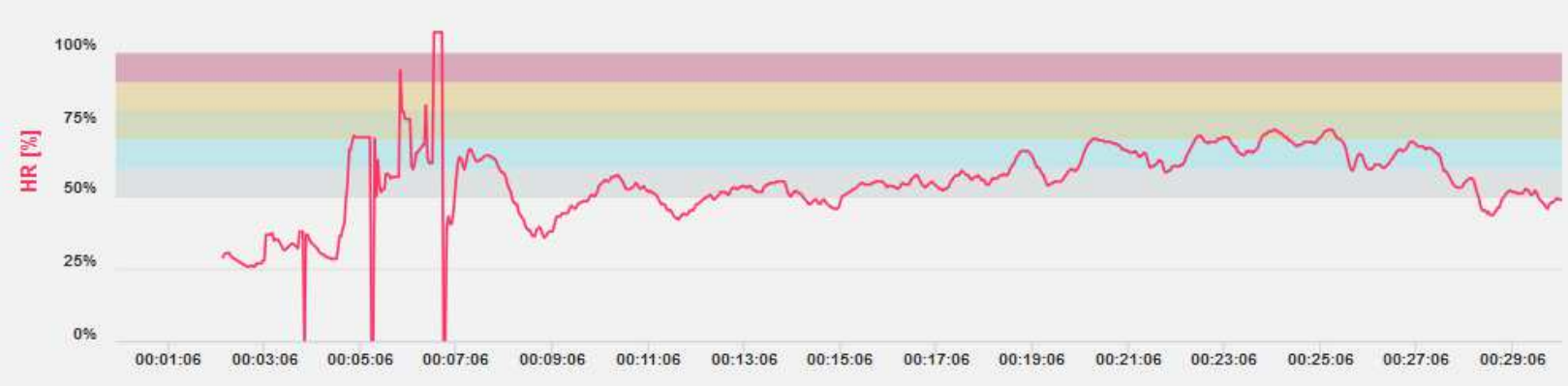

Figure B-47: 11/16/2018, session lasting 00:30:08, 1 occurrence lasting 00:00:12 peak of 203 (Artifact)

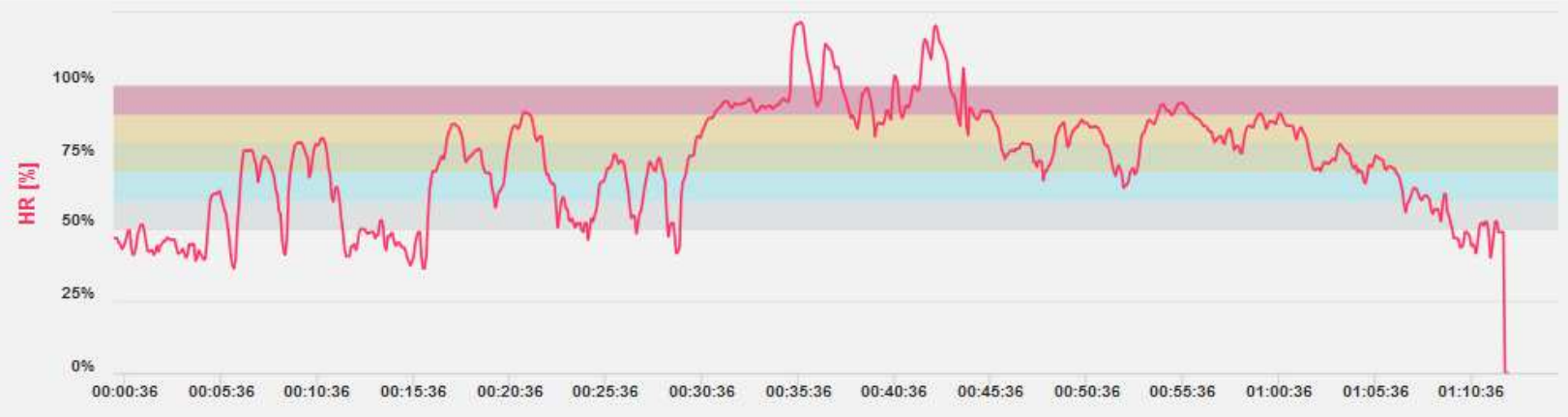

Figure B-48: 11/19/2018, session lasting 01:15:07, 1 occurrence lasting 00:09:08 peak of 231

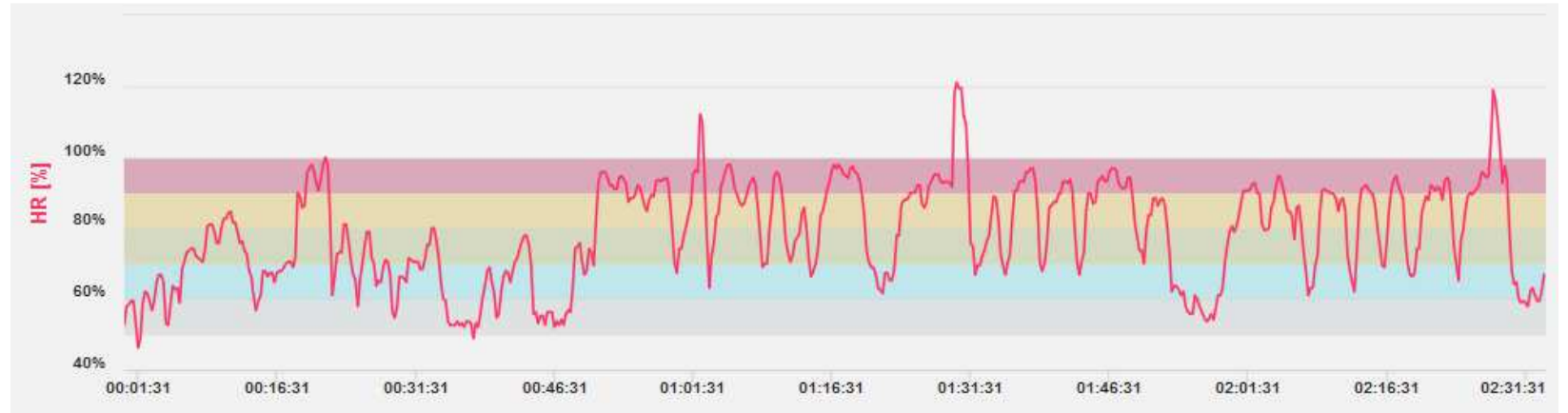

Figure B-49: 11/23/2018, session lasting 02:33:43, 3 occurrences lasting:

00:01:01 with peak of 213 00:01:44 with peak of 230 00:01:59 with peak of 226 


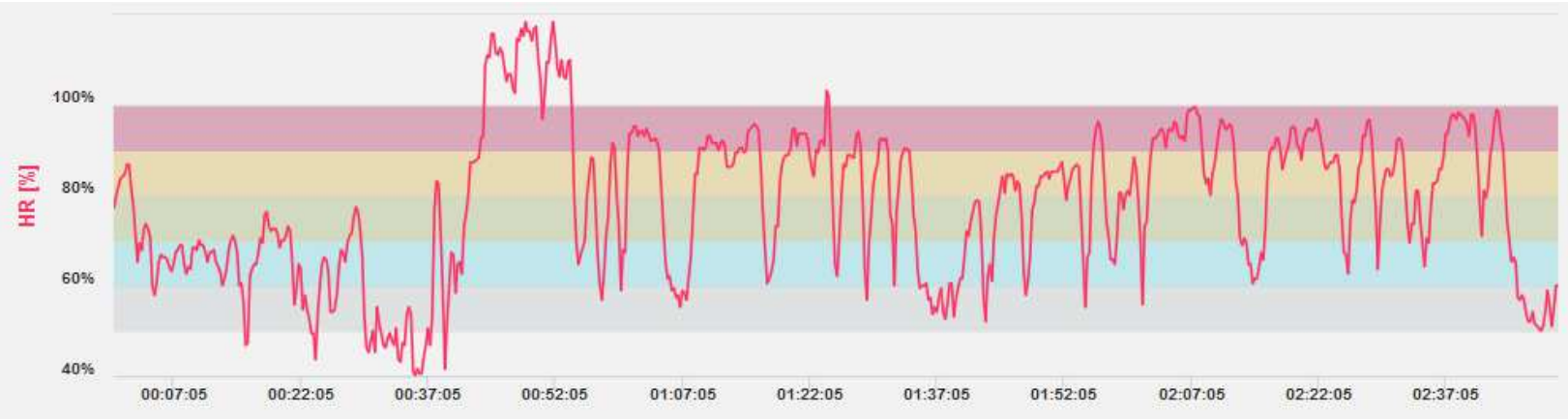

Figure B-50: 11/24/2018, session lasting 02:50:21, 1 occurrence that lasted 00:10:37 peak of 225

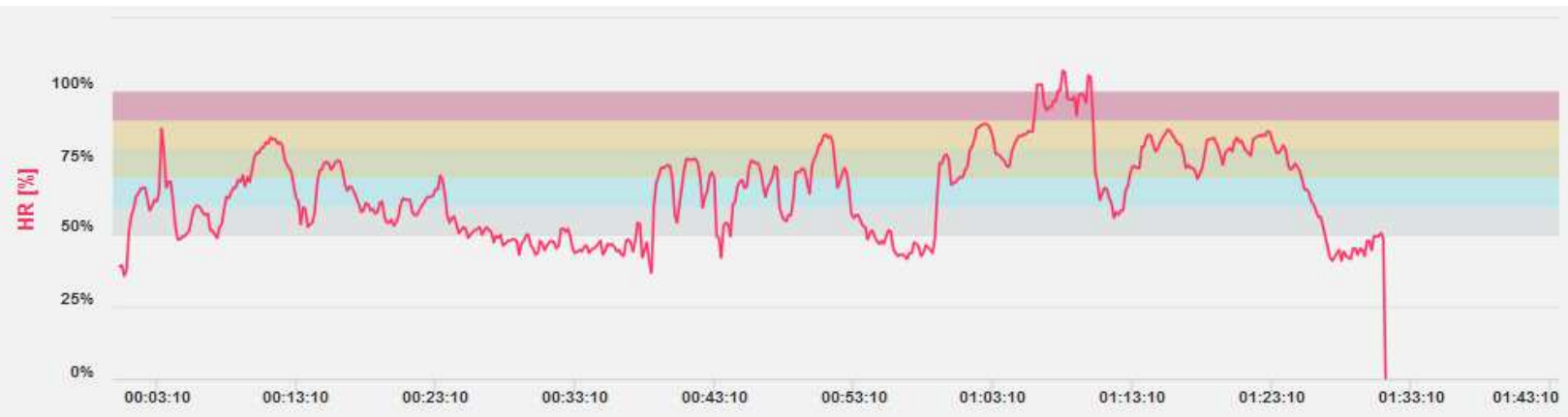

Figure B-51: 11/26/2018, session lasting 01:43:47, 1 occurrence that lasted 00:04:41 peak of 203

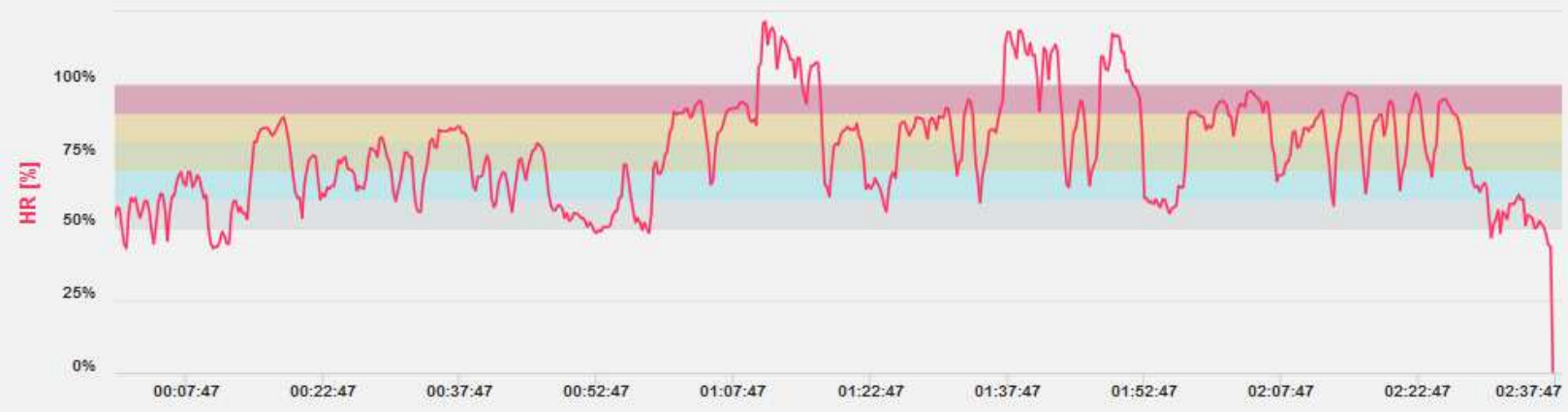

Figure B-52: 11/30/2018, session lasting 02:38:32, 3 occurrences lasting:

00:07:05 with peak of 231

00:06:31 with peak of 225

00:04:42 with peak of 223 


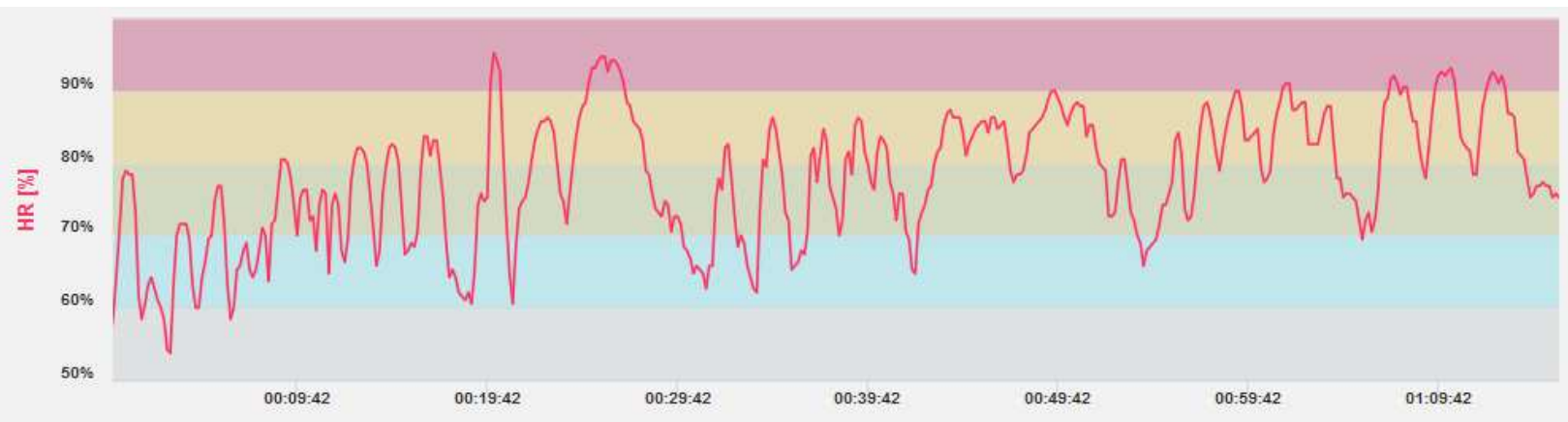

Figure B-53: 1/14/2019, session lasting 01:16:03, 1 occurrence that lasted 00:00:47 peak of 181

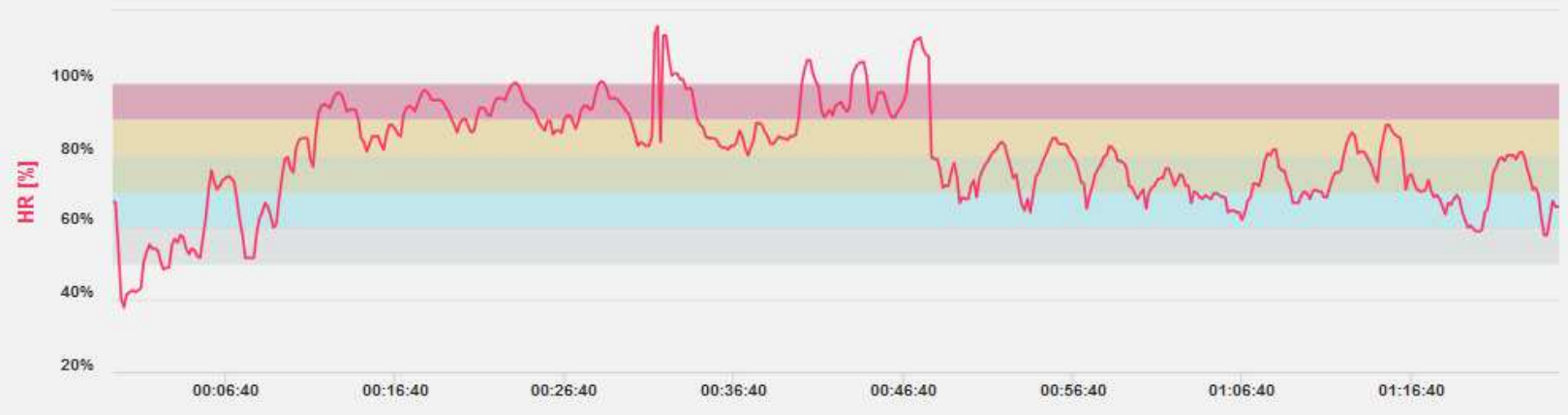

Figure B-54: 1/15/2019, session lasting 01:25:23 with 2 occurrences lasting:

00:01:48 with peak of 220

00:07:30 with peak of 214

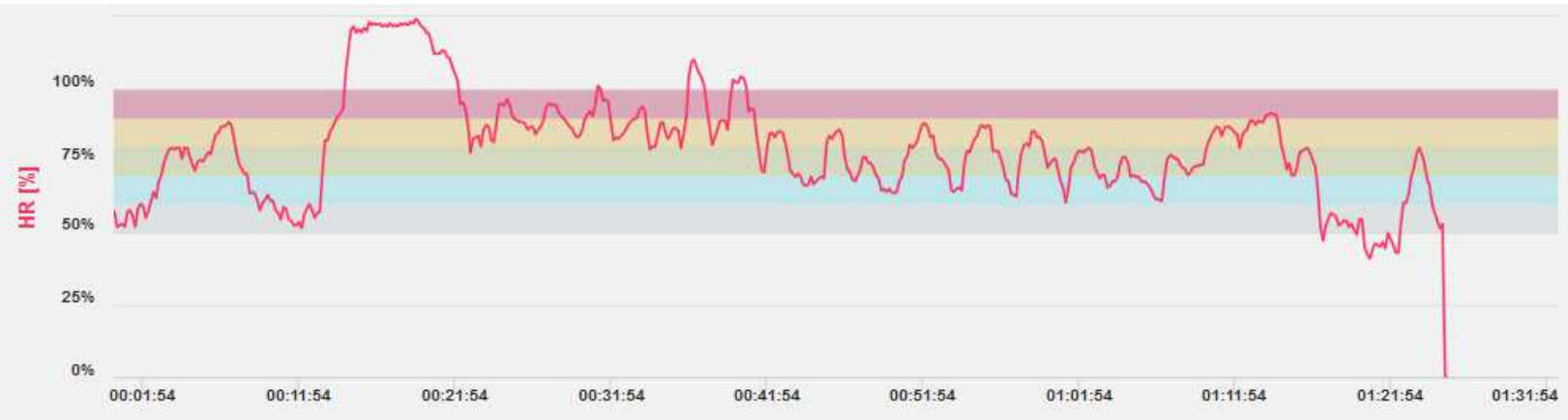

Figure B-55: 1/18/2019, session lasting 01:32:39, 1 occurrence lasting 00:26:29 peak of 236 


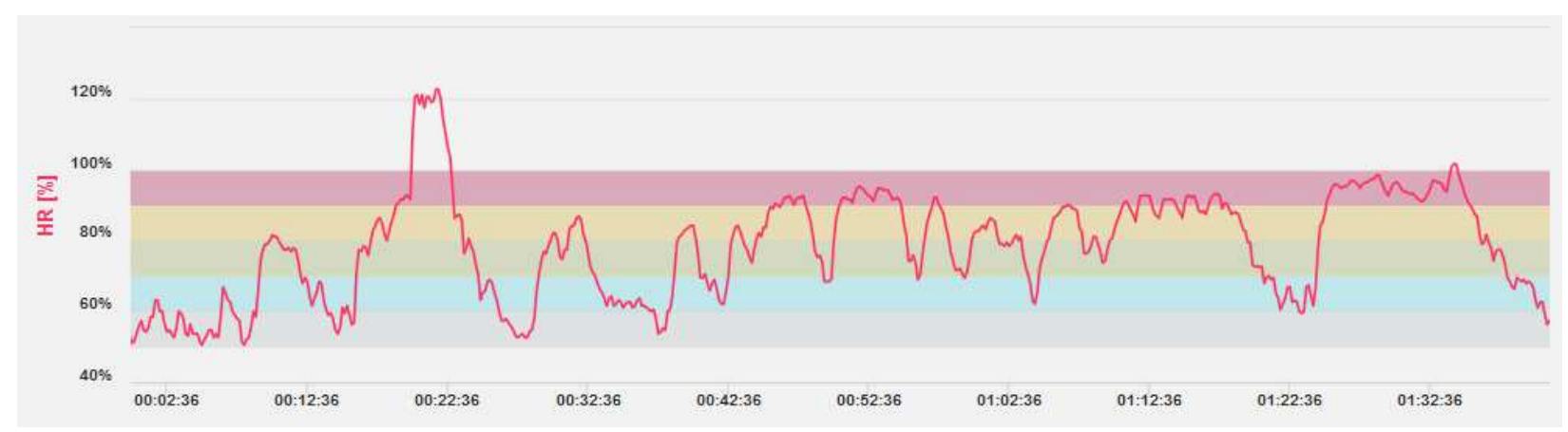

Figure B-56: 1/23/2019, session lasting 01:41:07, 1 occurrence lasting 00:03:40 peak of 233

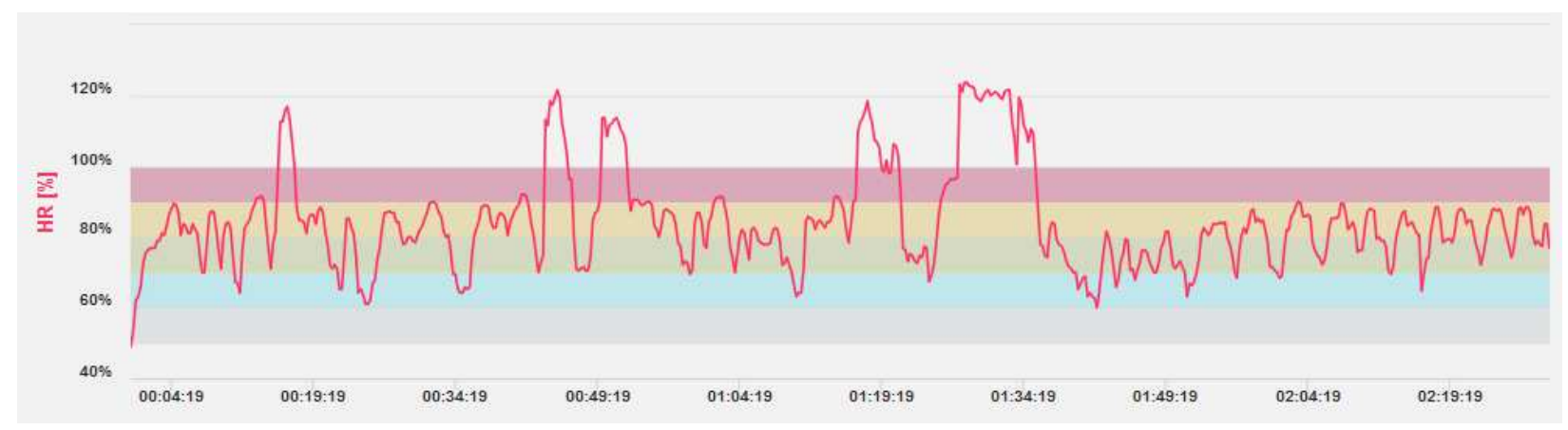

Figure B-57: 1/28/2019, session lasting 02:29:52, 5 occurrences lasting:

00:02:03 with peak of 222

00:03:08 with peak of 231

00:02:57 with peak of 216

00:04:43 with peak of 225

00:08:12 with peak of 235

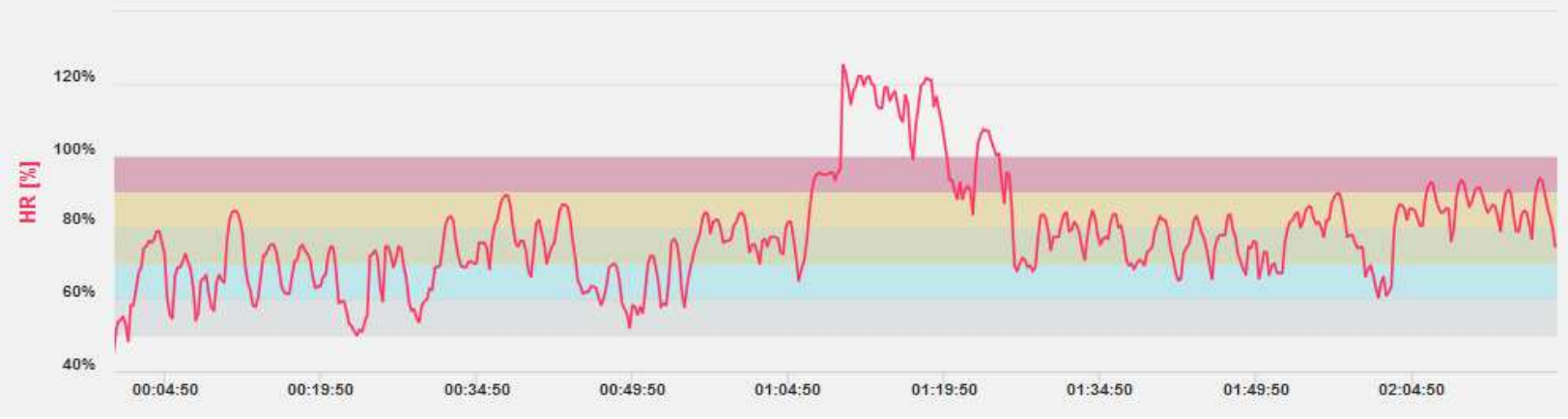

Figure B-58: 1/29/2019, session lasting 02:18:47, 1 occurrence lasting 00:10:03 peak of 237 


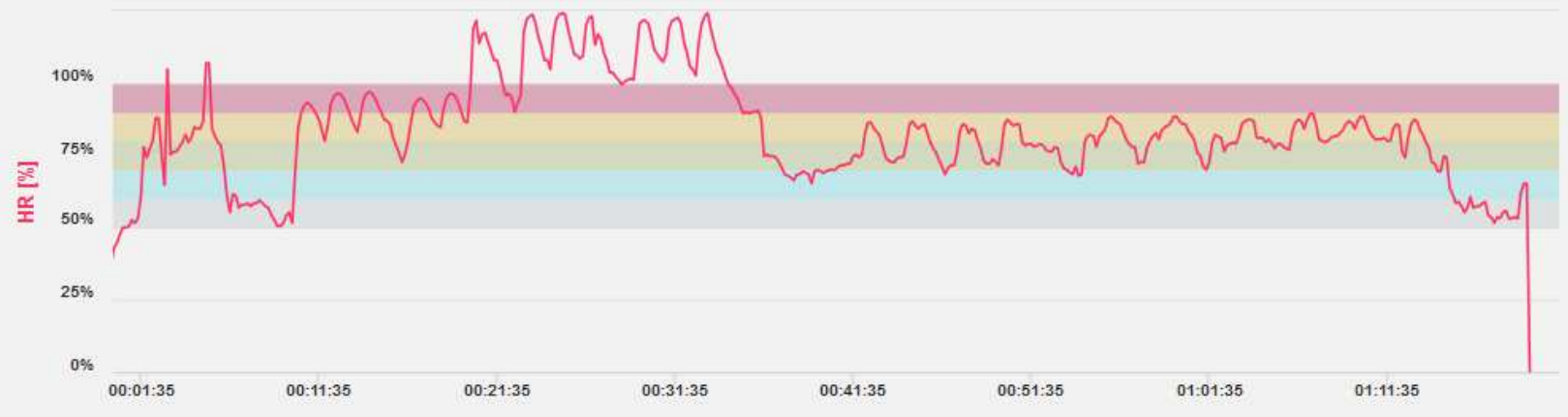

Figure B-59: 1/31/2019, session lasting, 01:21:14, loccurrence lasting 00:16:27 peak of 236

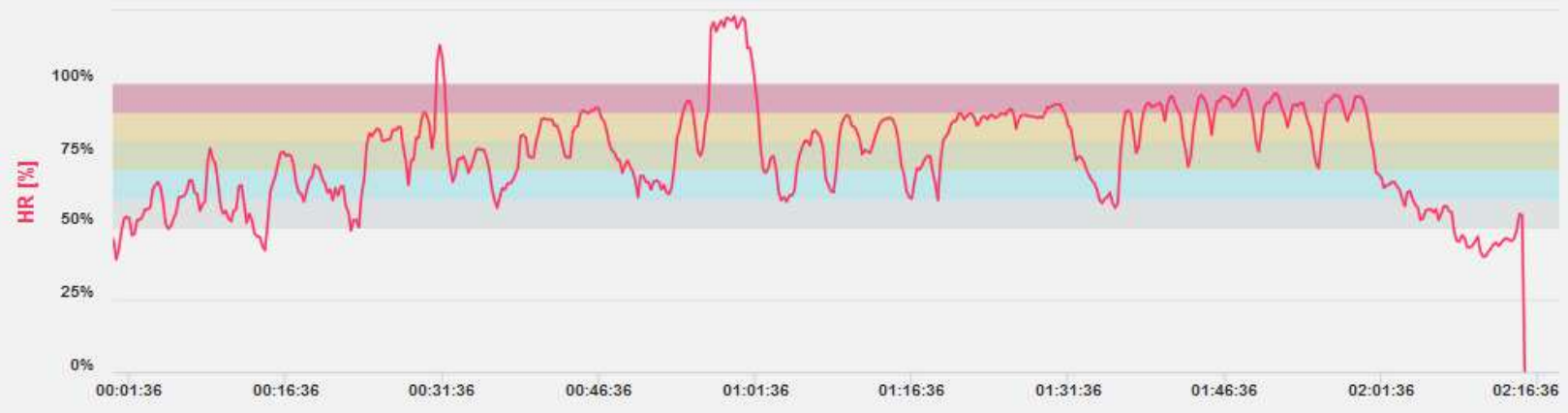

Figure B-60: 2/1/2019, session lasting 02:18:39, 2 occurrences lasting:

00:01:15 with peak of 216 00:04:42 with peak of 234

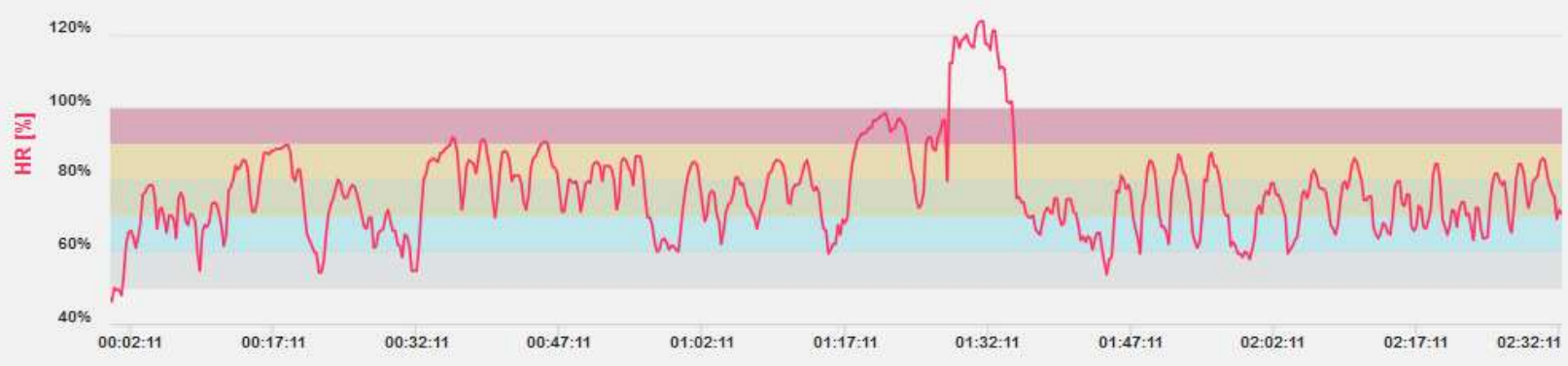

Figure B-61: 2/4/2019, session lasting 02:32:29, 1 occurrence lasting 00:06:53 peak of 235 


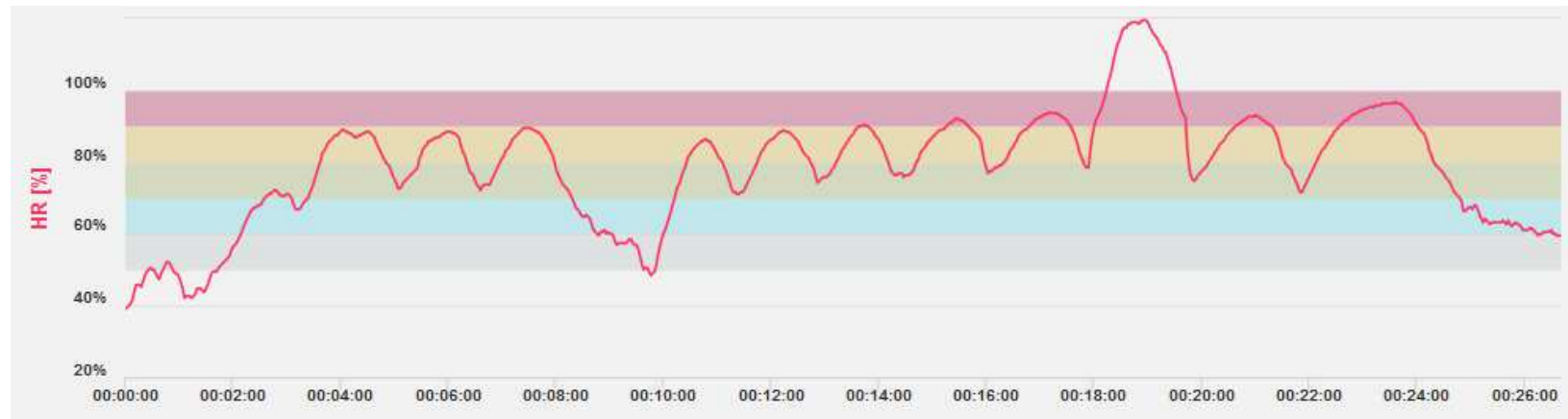

Figure B-62: 2/7/2019, session lasting 00:26:41, 1 occurrence lasting 00:01:56 peak of 227, this figure was while subject was wearing Holter monitor

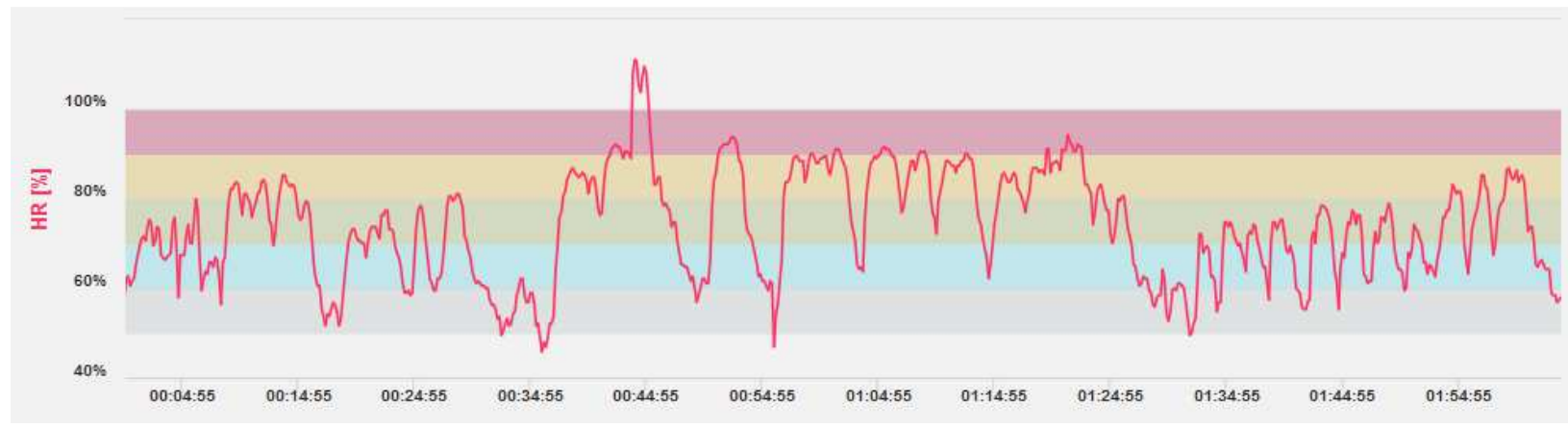

Figure B-63: 2/11/2019, session lasting 02:03:46, 1 occurrence lasting 00:01:47 peak of 211

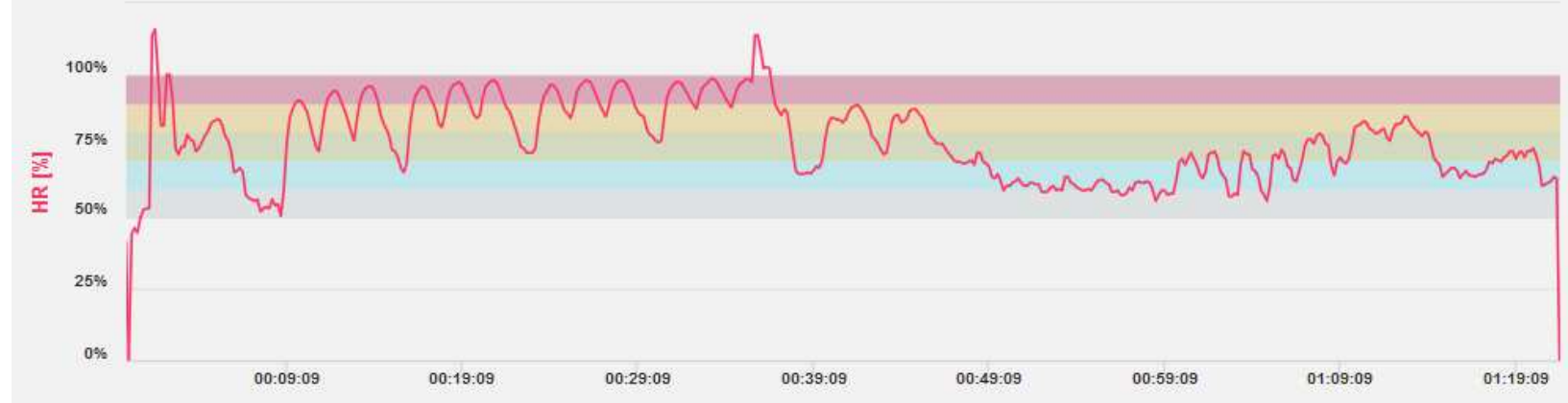

Figure B-64: 2/15/2019, session lasting 01:21:41, 2 occurrences lasting

00:00:13 with peak of 220

00:02:18 with peak of 216 


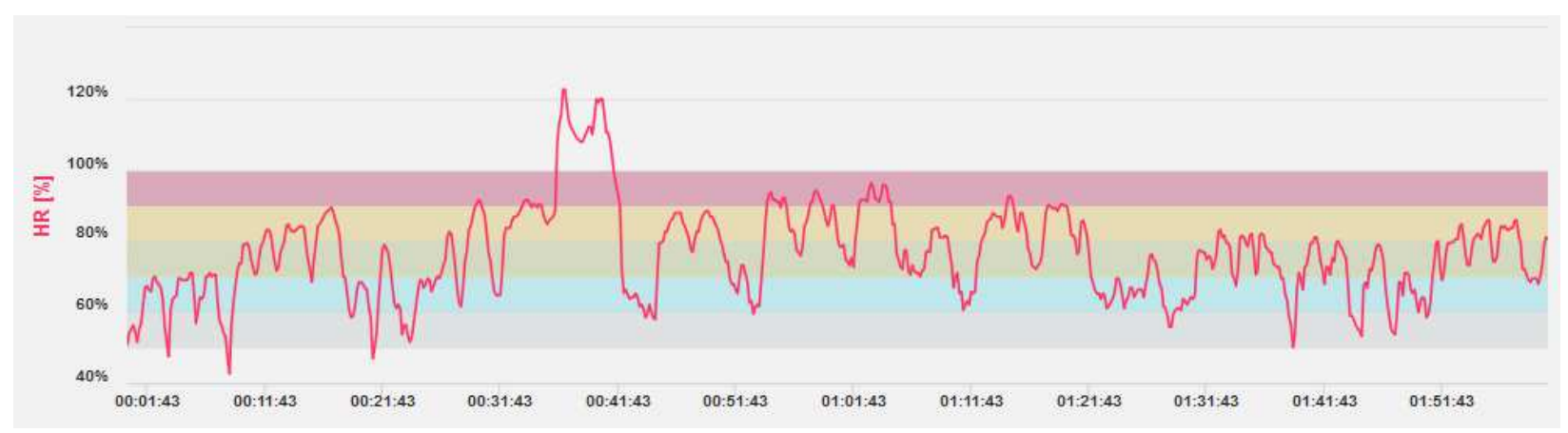

Figure B-65: 2/18/2019, session lasting 02:00:42, 1 occurrence lasting 00:05:27 peak of 233

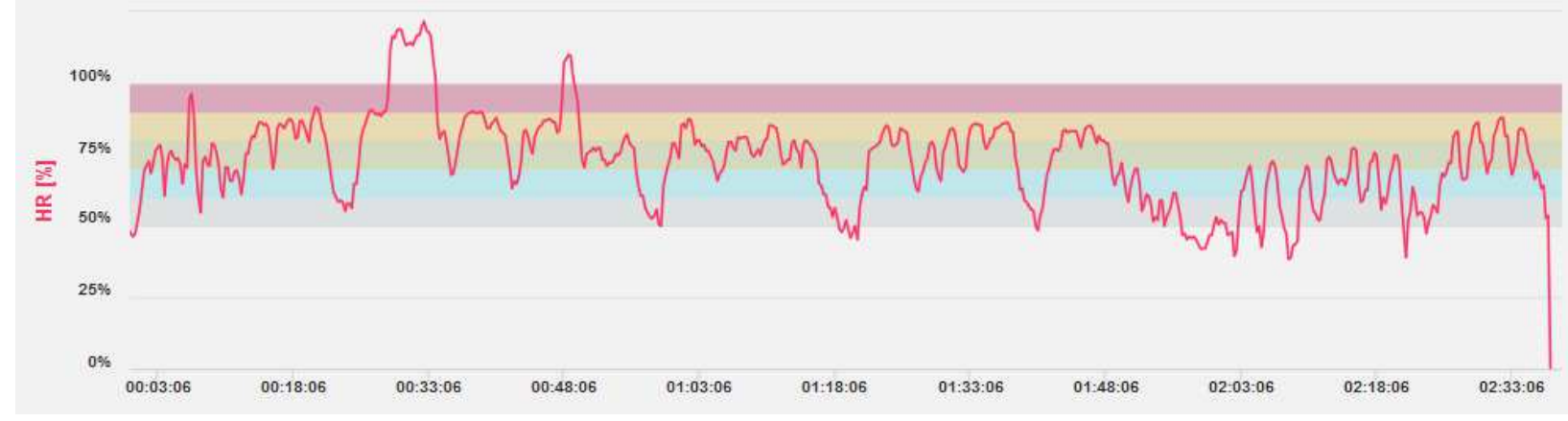

Figure B-66: 2/28/2019, session lasting 02:38:39, 3 occurrences lasting:

00:00:44 with peak of 183 (submaximal)

00:05:30 with peak of 231

00:02:17 with peak of 209

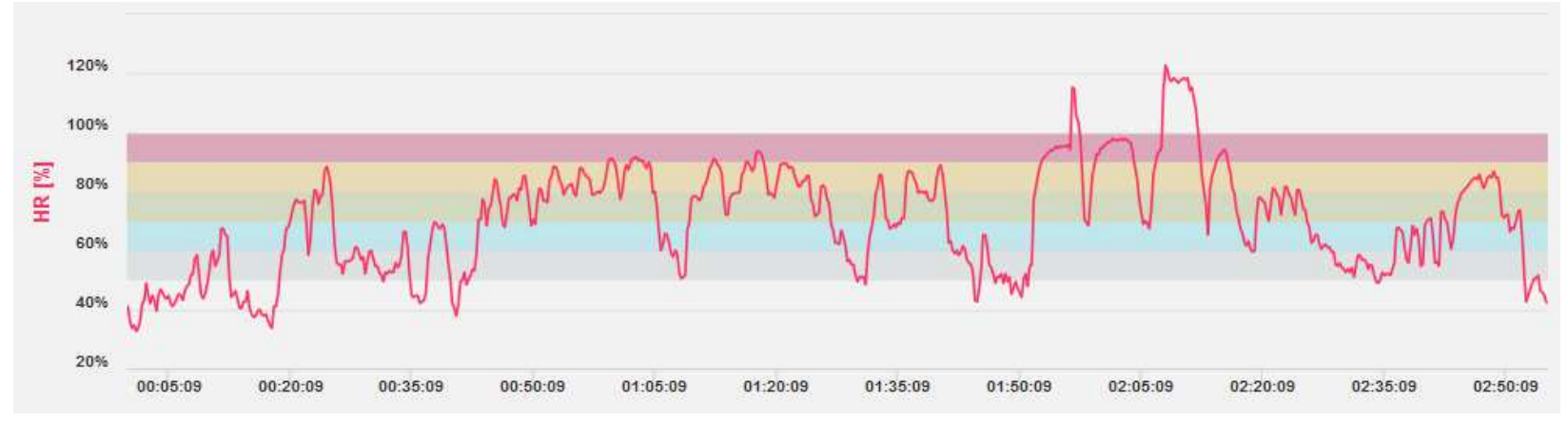

Figure B-67: 3/1/2019, session lasting 02:55:20, 2 occurrences lasting:

00:01:35 with peak of 219

00:05:21 with peak of 231 


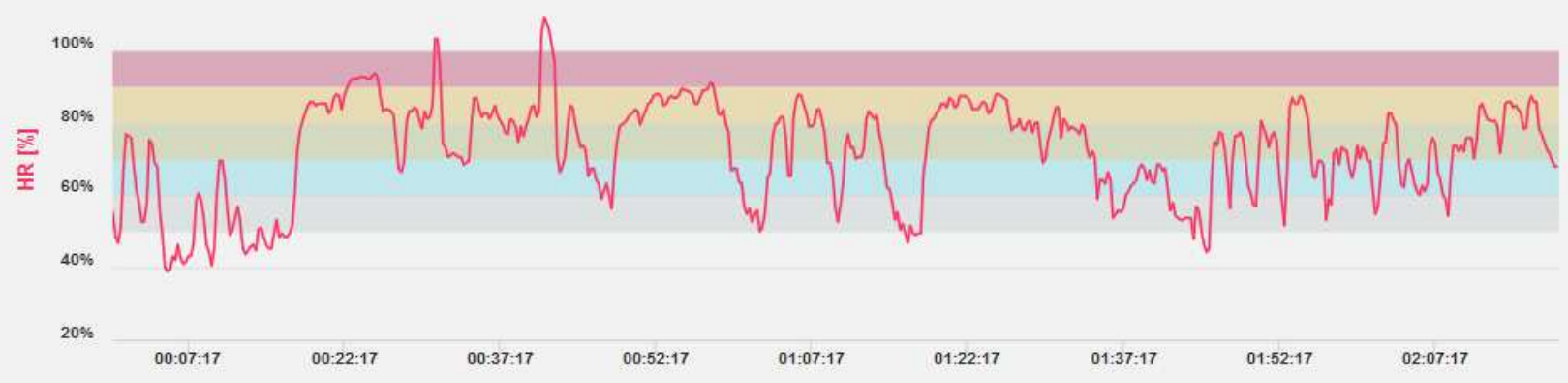

Figure B-68: 3/4/2019, session lasting 02:19:13, with 2 occurrences lasting:

00:00:33 with peak of 196

00:01:34 with peak of 207

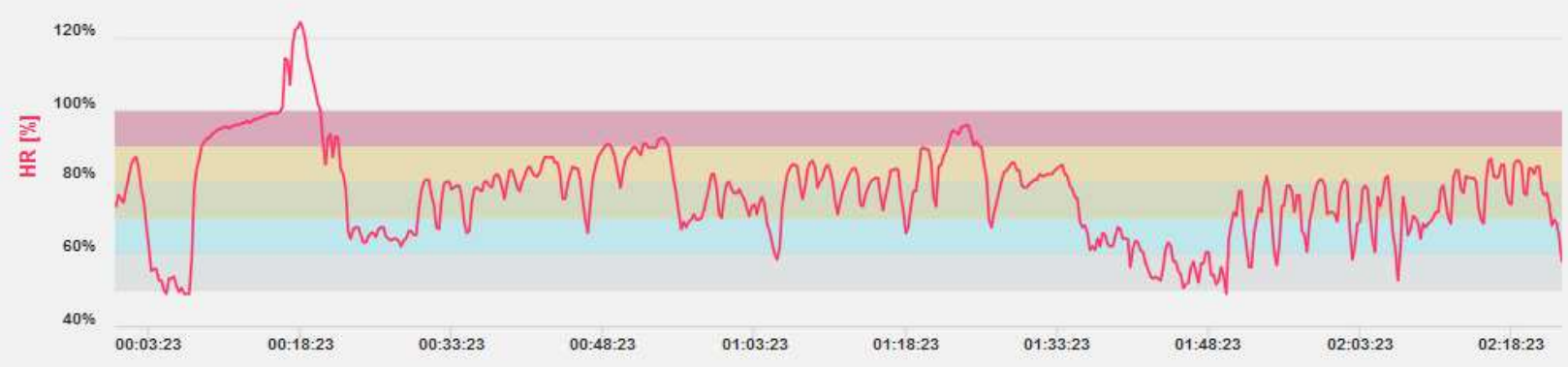

Figure B-69: 3/7/2019, session lasting 02:23:24, with 1 occurrence lasting 00:06:37 peak of 236

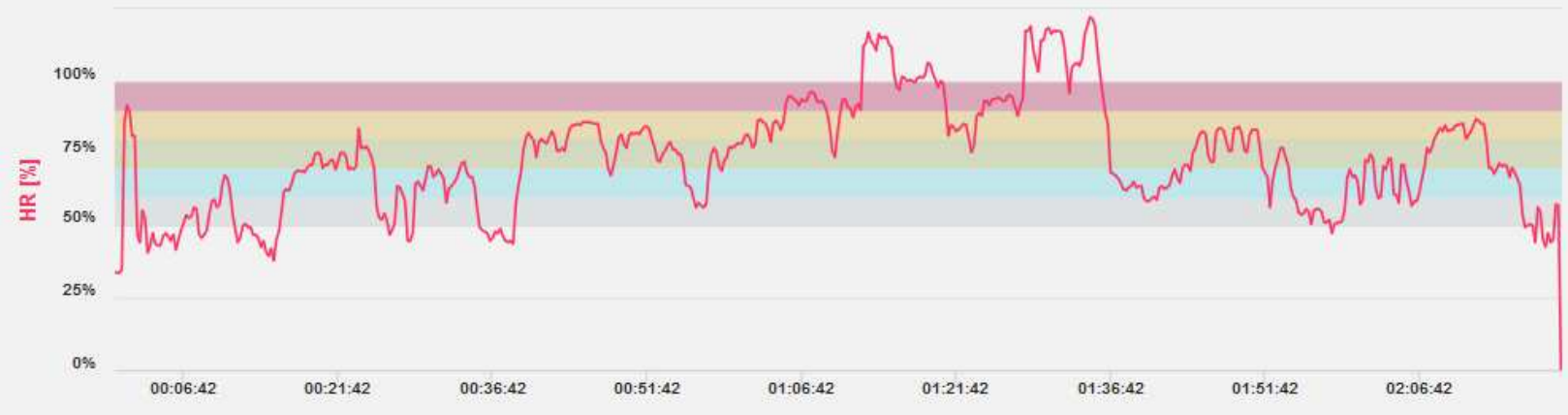

Figure B-70: 3/8/2019, session lasting 02:20:33, with 2 occurrences lasting:

00:08:08 with peak of 222

00:08:26 with peak of 227 


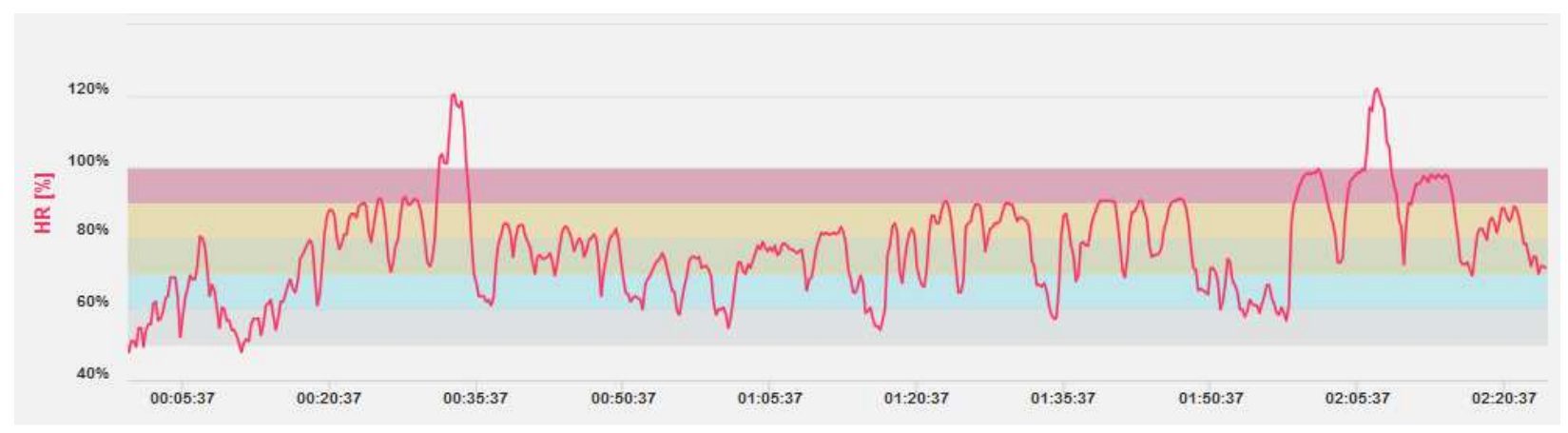

Figure B-71: 3/19/2019, session lasting 02:25:05, with 2 occurrences lasting:

00:03:28 with peak of 229

00:03:20 with peak of 231

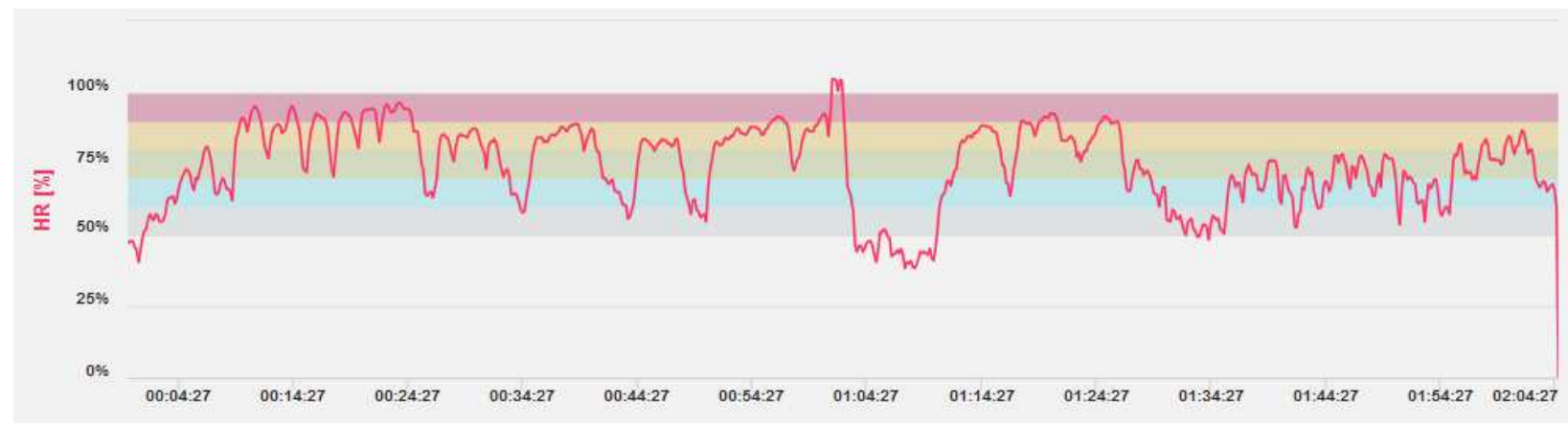

Figure B-72: 4/1/2019, session lasting 02:04:46, with 1 occurrence lasting 00:01:27 peak of 199

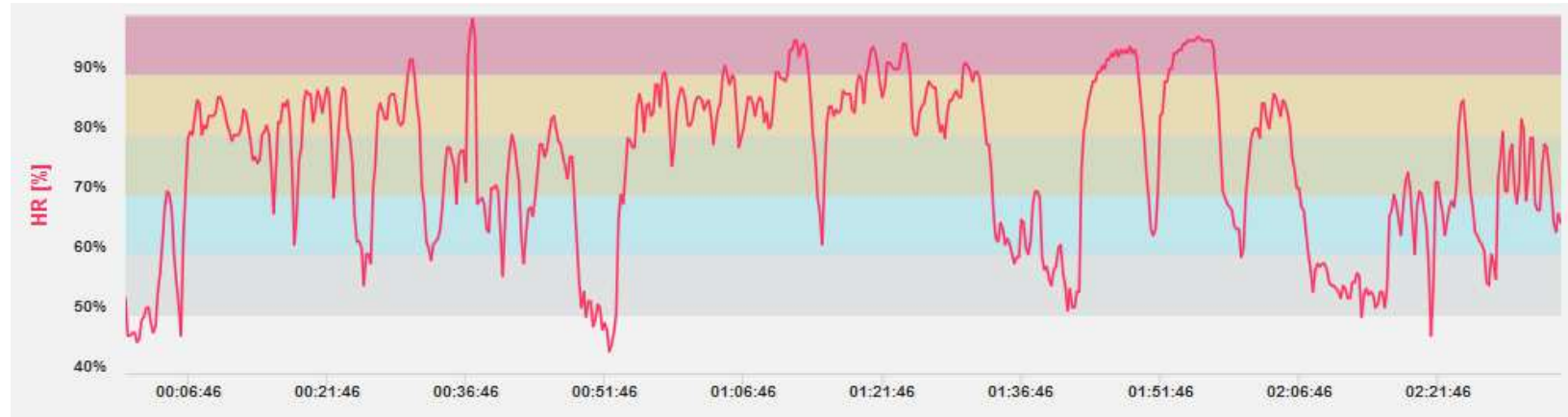

Figure B-73: 4/9/2019, session lasting 02:35:04, with 1 occurrence lasting 00:01:01 peak of 189 


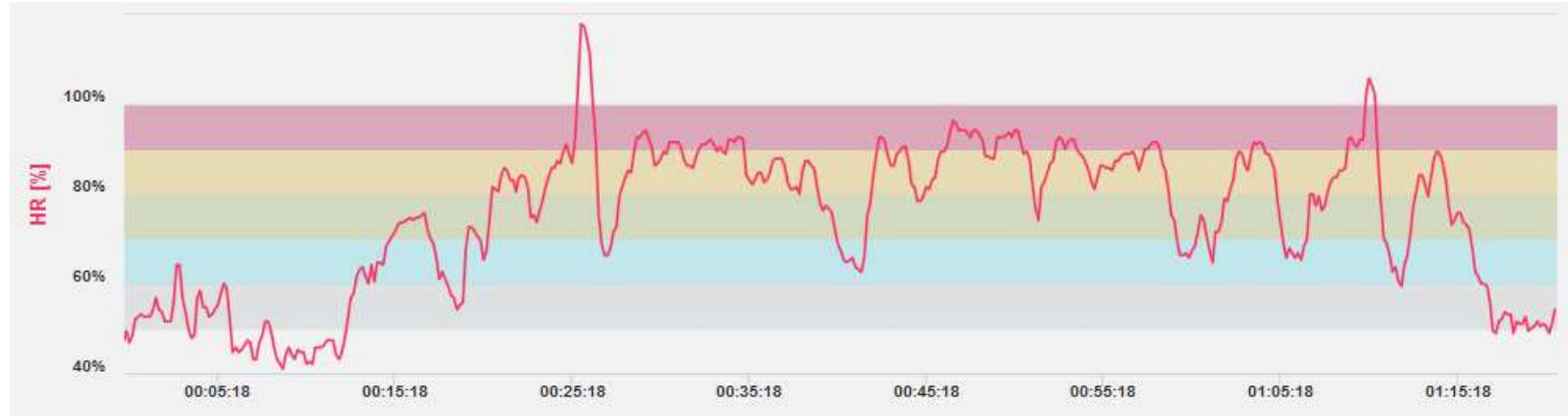

Figure B-74: 4/13/2019, session lasting 01:20:56, with 2 occurrences lasting

00:01:10 with peak of 224

00:01:01 with peak of 201

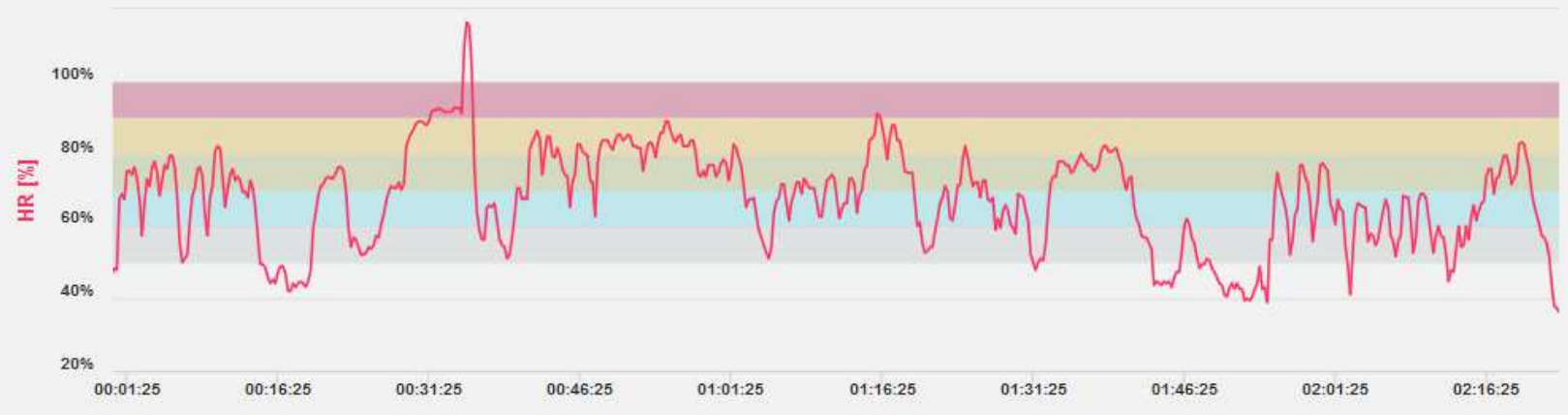

Figure B-75: 4/15/2019, session lasting 02:23:39 with 1 occurrence lasting 00:01:07 peak of 221

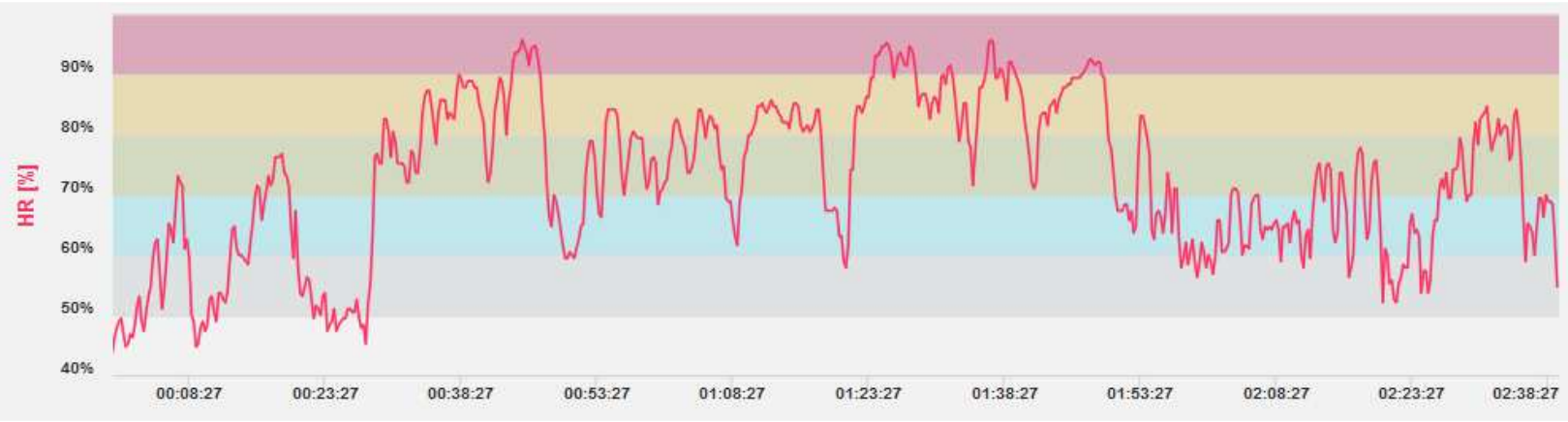

Figure B-76: 4/18/2019, session lasting 02:39:40 with 1 occurrence lasting 00:00:11 peak of 128 


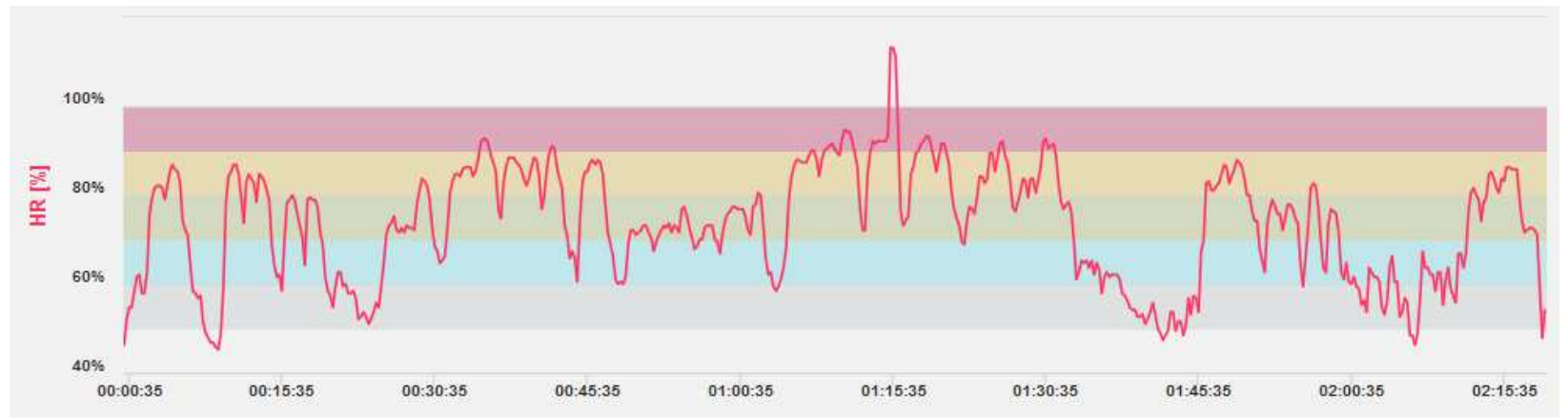

Figure B-77: 4/22/2019, session lasting 02:19:47 with 1 occurrence lasting 00:00:57 peak of 215

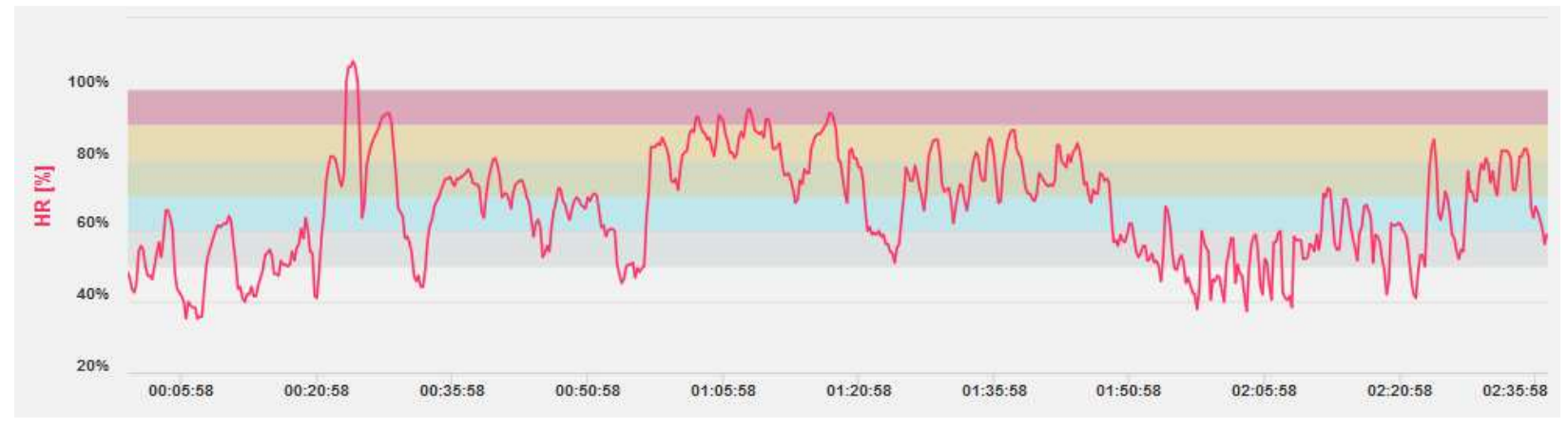

Figure B-78: 4/23/2019, session lasting 02:37:20 with 1 occurrence lasting 00:01:44 peak of 205

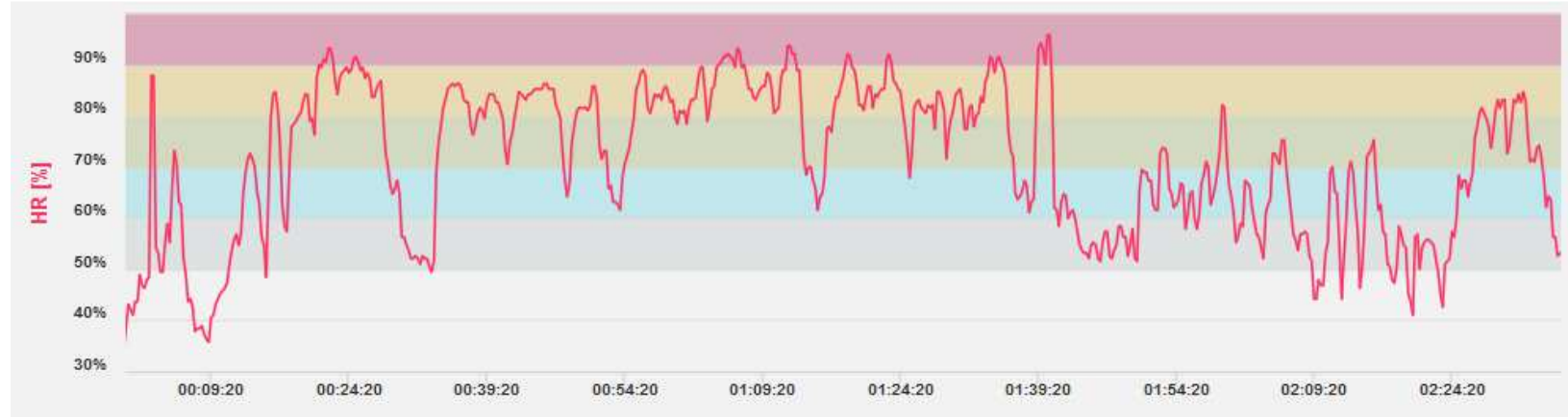

Figure B-79: 4/25/2019, session lasting 02:36:13 with 1 occurrence lasting 00:01:58 peak of 182 


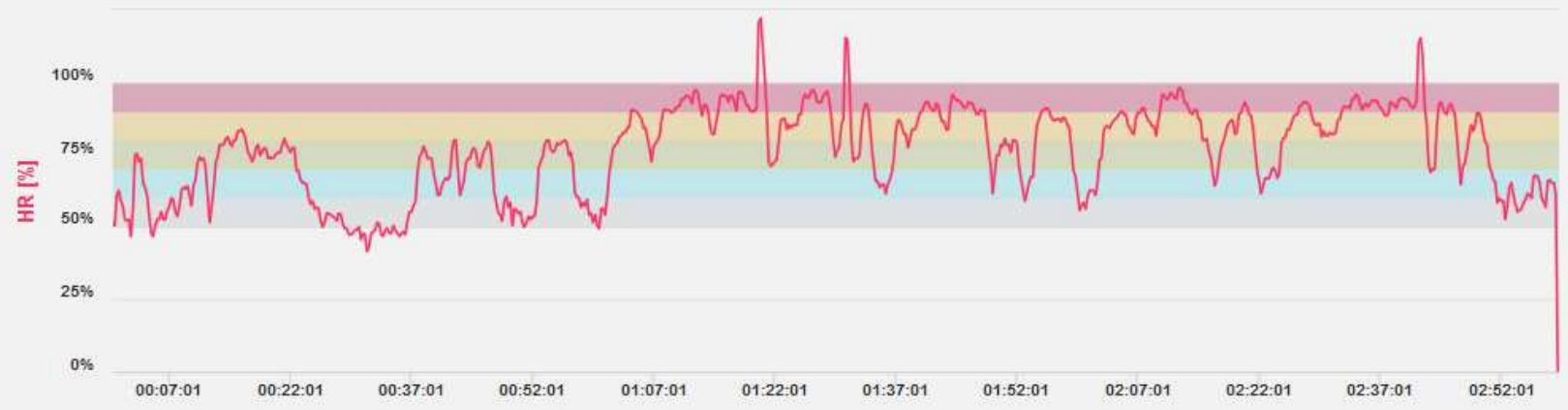

Figure B-80: 4/26/2019, session lasting 02:59:11 with 3 occurrences lasting 00:01:20 with peak of 232

00:00:53 with peak of 219

00:00:51 with peak of 219 


\section{APPENDIX C: NUMERIC ANALYSIS OF “ARRHYTHMIA” DATA}

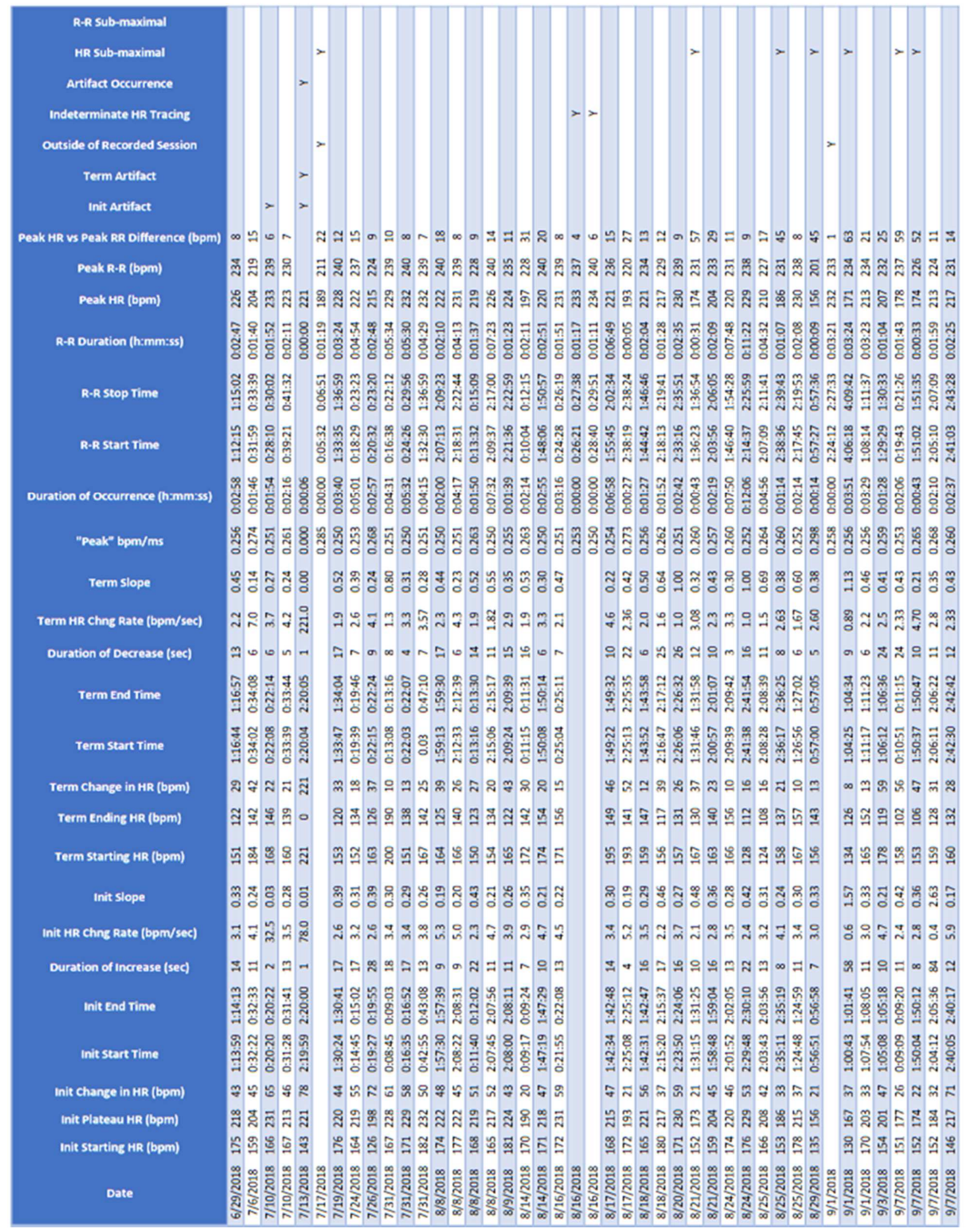




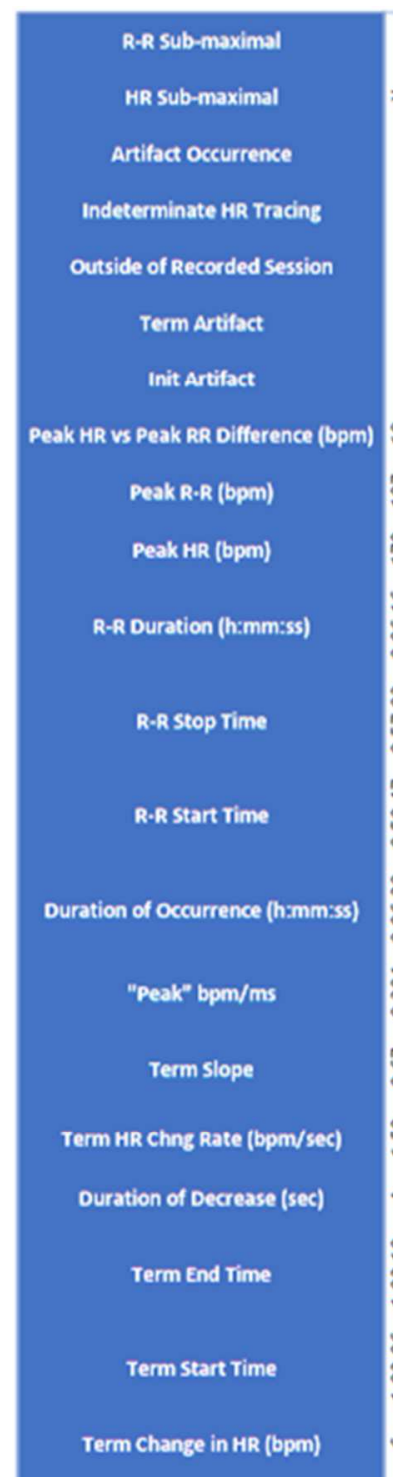

Term Ending HR (bpm)

Term Starting HR (bpm)

Init slope

Init HR chns Rate (bpm/sec)

Duration of Increase (sec)

Init End Time

Init start Time

Init change in HR (bpm)

Init Plateau HR (bpm)

Init Starting HR (bpm)

Date

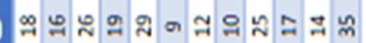

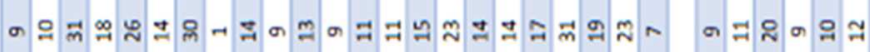

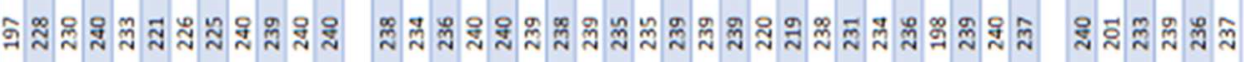

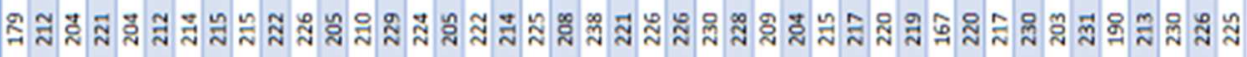
๑ำ

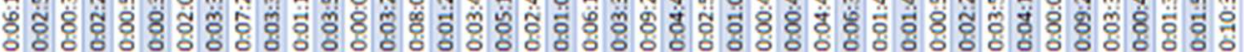

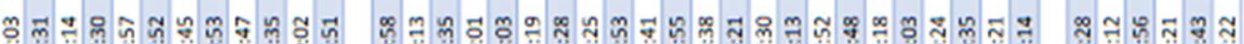

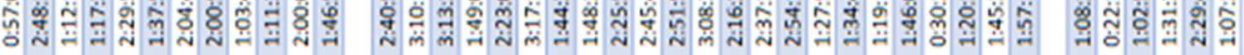

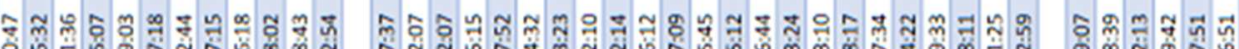

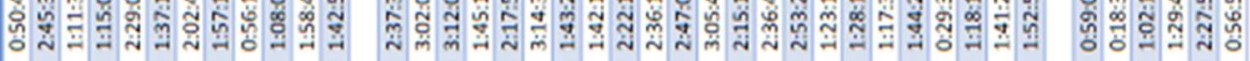

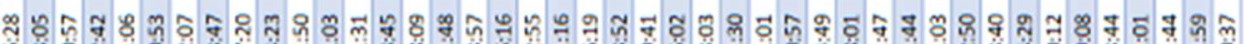

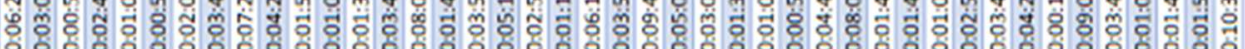
-

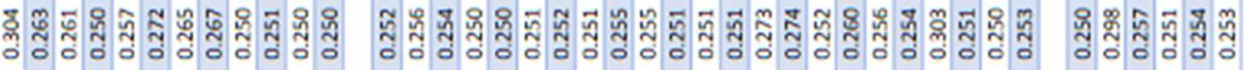

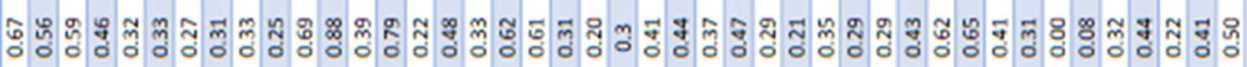

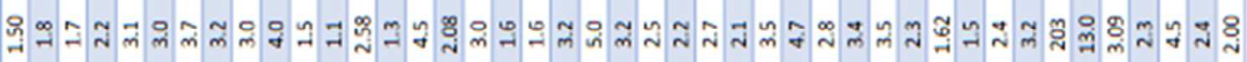
ข 운

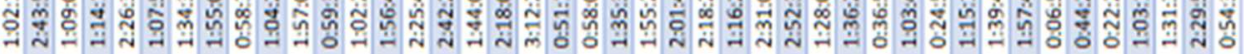

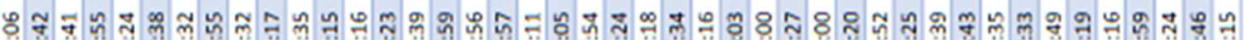

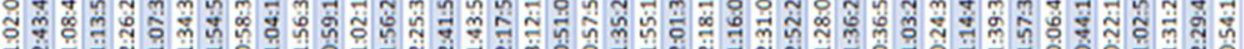
n-n

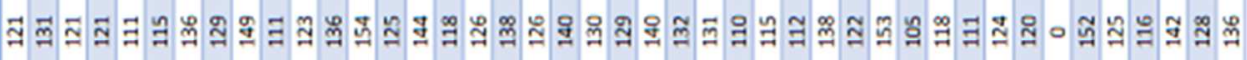

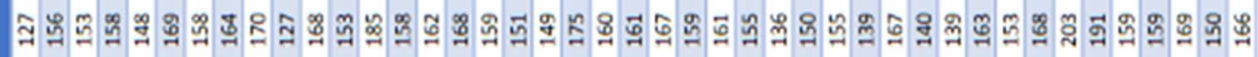

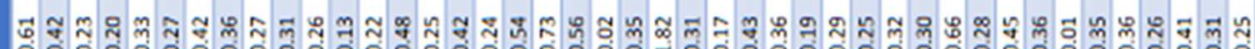

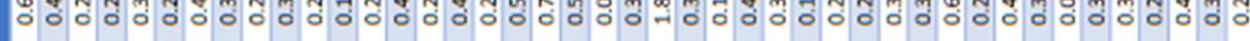

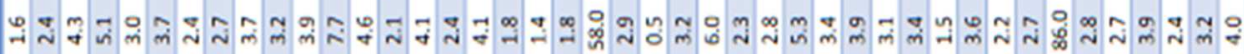
ทุำ ธิธิ윽ㄱㄱำ

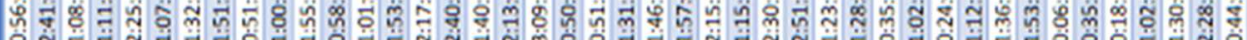

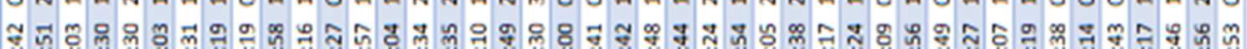

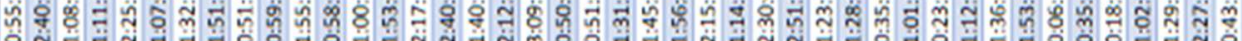

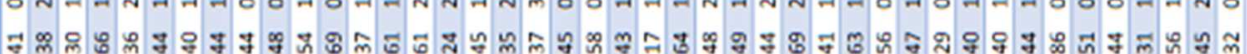

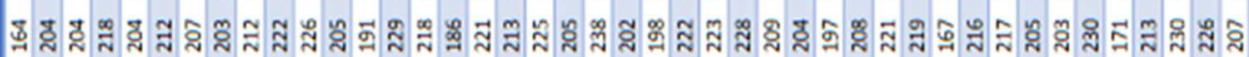

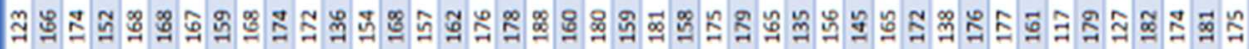
胥范 สু 


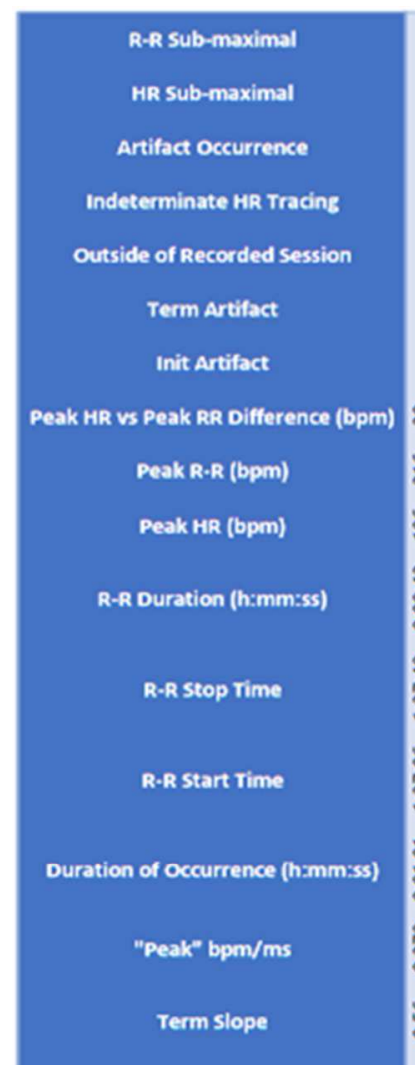

Term HR chng Rate (bpm/sec)

Duration of Decrease (sec)

Term End Time

Term start Time

Term change in HR (bpm)

Term Ending HR (bpm)

Term Starting HR (bpm)

Init Slope

Init HR Chns Rate (bpm/sec)

Duration of Increase (sec)

Init End Time

Init start Time

Init change in HR (bpm)

Init Plateau HR (bpm)

Init starting HR (bpm)

Date
ภิ

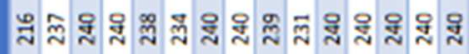
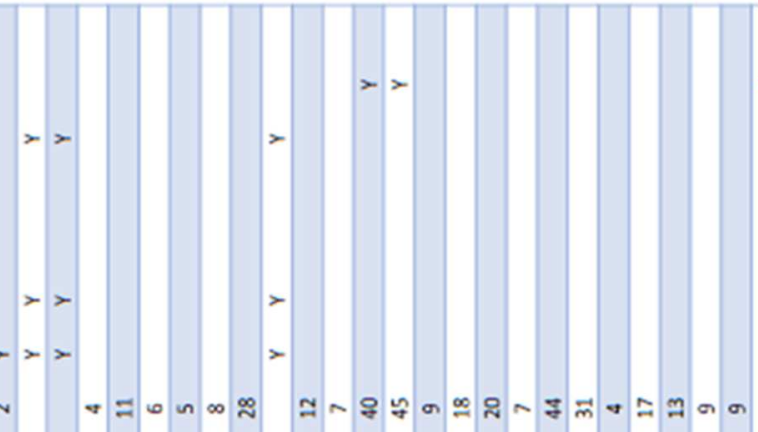

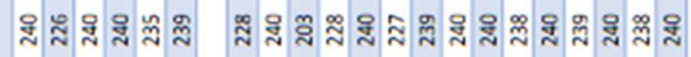

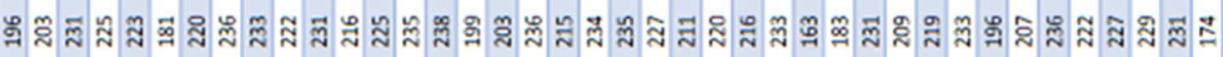

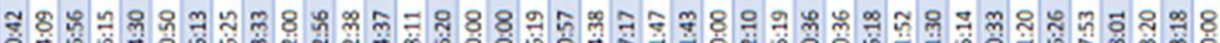

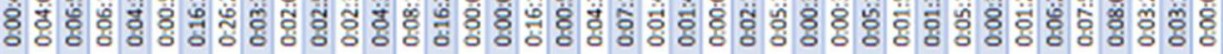

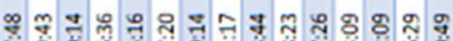

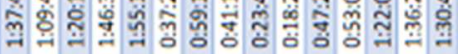

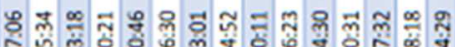

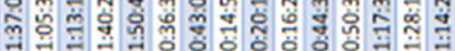

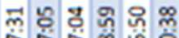

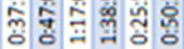

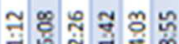

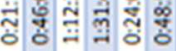

离寻哭设

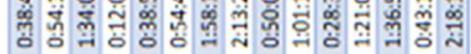

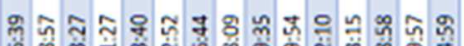

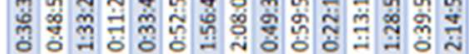

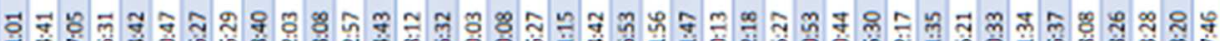

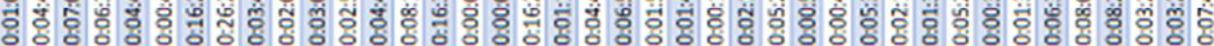

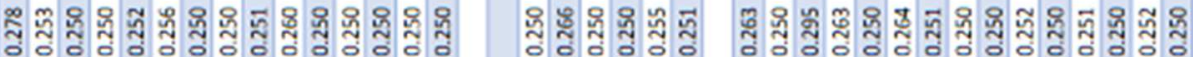
年

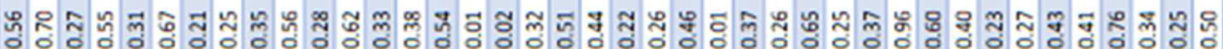

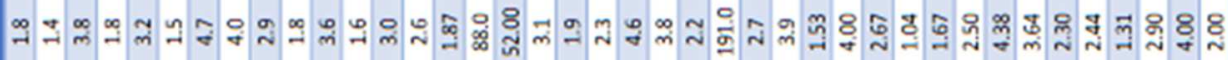

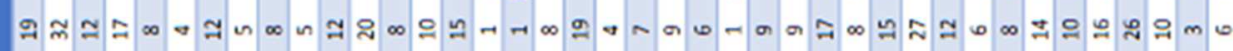

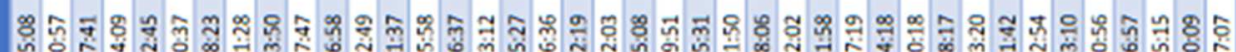

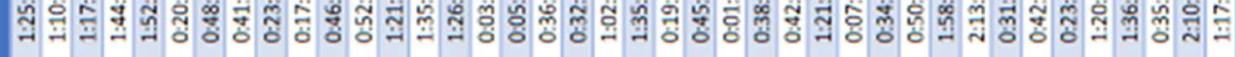
จำกำกิ

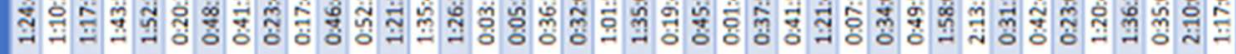

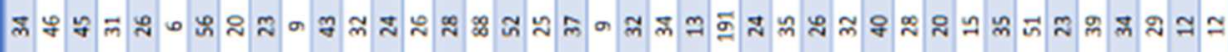

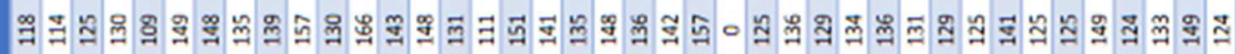

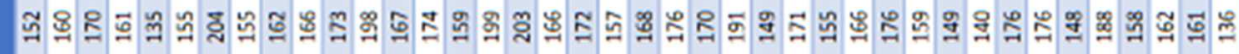

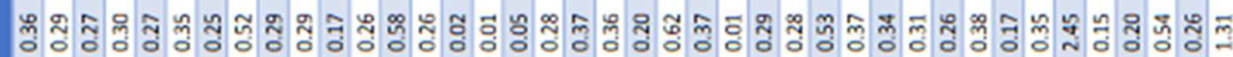

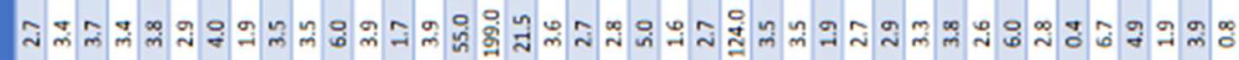

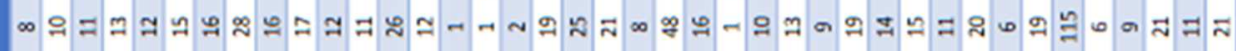

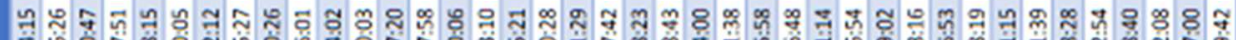

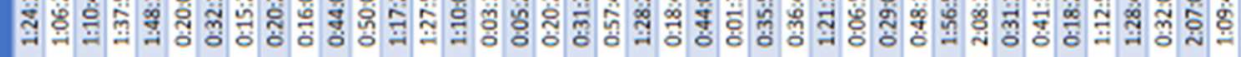

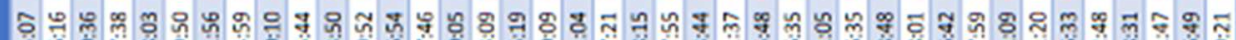

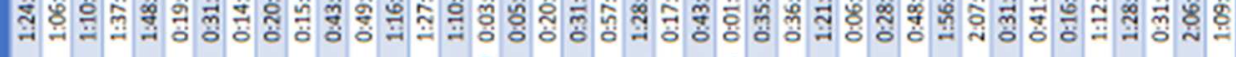

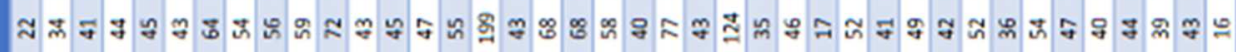

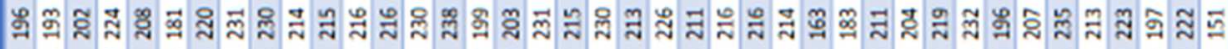

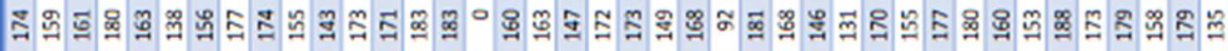

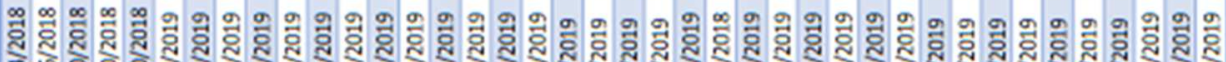

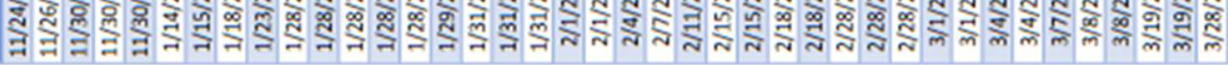

Figure C-1: Numerical analysis of "arrhythmia" data 


\section{APPENDIX D: DESCRIPTION OF COLUMNS FOUND IN TABLE 1}

Date: Self-explanatory, input from date located on recorded session.

Init Starting HR (bpm): This is the HR at the initial onset of the observed occurrence. This was determined by physically placing the cursor on a point that was visually determined to be a marked departure in HR. The number indicated is the HR at that time

Init Plateau HR (bpm): This is the HR at the first plateau reached after initial HR increase. This is not the peak HR of the occurrence, but is simply the beginnings of what could be considered an elevated "steady-state" for the duration of the occurrence. This was determined by placing the cursor at a point after initial HR increase where no change was indicated by a horizontal line on the HR tracing.

Init Change in HR (bpm): This is the amount of the initial increase in HR as determined by subtracting the Init Plateau HR from the Init Starting HR

Init Start Time: Time that occurrence began, information gathered the same way as Init Starting HR

Init End time: Time that initial HR acceleration plateaued, information gathered the same way as Init Plateau HR

Duration of Increase (Sec): This is the amount of time that the initial increase of the occurrence took. This was determined by subtracting the Init End Time from the Init Start Time

Init HR Chng Rate (bpm/sec): This is the rate of change of HR during the initial occurrence increase. This value was determined by dividing Init Change in HR (bpm) by Duration of Increase $(\mathrm{Sec})$

Init Slope: Determined by dividing Duration of Increase (Sec) by Init Change in HR (bpm). This is used as the criteria for determining if an initiation of a possible occurrence is artifact. 
Term Starting HR (bpm): This is the HR at the start of the termination of the observed occurrence. This was determined by physically placing the cursor on a point that was visually determined to be a marked return to normal HR. The number indicated is the HR at that time. Term Ending HR (bpm): This is the HR at the first plateau reached after the termination of the occurrence has started. This point was determined by visually observing the HR trace to see where no change in HR was observed and that the subject had returned to a level that would be considered commensurate with other athletes performing the same drill/exercise. This was determined by placing the cursor at a point after HR decrease where no change was indicated by a horizontal line on the HR tracing.

Term Change in HR (bpm): This is the amount of decrease in HR as determined by subtracting the Term Ending HR from the Term Starting HR

Term Start Time: This is the time of the start of the termination of the occurrence. Determined in the same manner as the Term Starting HR.

Term End Time: This is the time when the first plateau is reached after the termination of the occurrence has started. Determined in the same manner as the Term Ending HR.

Duration of Decrease (sec): This is the amount of time that the termination portion of the occurrence took. This was determined by subtracting the Init End Time from the Init Start Time

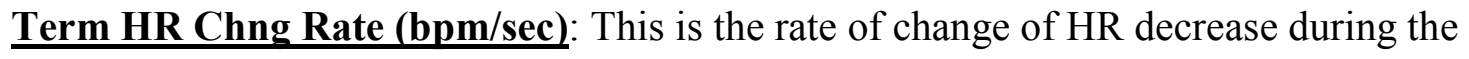
termination portion of the occurrence. This value was determined by dividing Term Change in HR (bpm) by Duration of Decrease (Sec)

Term Slope: This is used as the criteria for determining if a termination of a possible occurrence is artifact. Determined by dividing Duration of Decrease (Sec) by Term Change in HR (bpm). 
"Peak" bpm/ms: This number is a measure of the time between " $\mathrm{R}$ " waves in the cardiac cycle. This R-R value can be used to determine. Determined by placing cursor on "lowest" point during an occurrence in the Polar software. This number is then converted into milliseconds for conversion into an actual HR.

Duration of Occurrence (h:mm:ss): This column provides for total time that the occurrence took place. For calculating this variable, subtract Init Start Time from Term End Time R-R Start Time: This value will serve as the beginning point of the occurrence. This value is determined by placing the cursor on the RR Interval analysis screen where an occurrence is observed (placing the cursor where the blue arrow is indicating would provide the R-R Start Time). Placing the cursor at the last point before initiation of the occurrence, usually indicated by an extremely abrupt departure from steady state (Figure D-1).

R-R Stop Time: This value will serve as the actual termination of the occurrence. This value is determined by placing the cursor on the RR Interval analysis screen where RRI is observed to be returning to "pre-occurrence" levels. For this subject it is often signaled by a steadily increasing variability in RRI followed by an abrupt return to pre occurrence levels (Figure D-2).

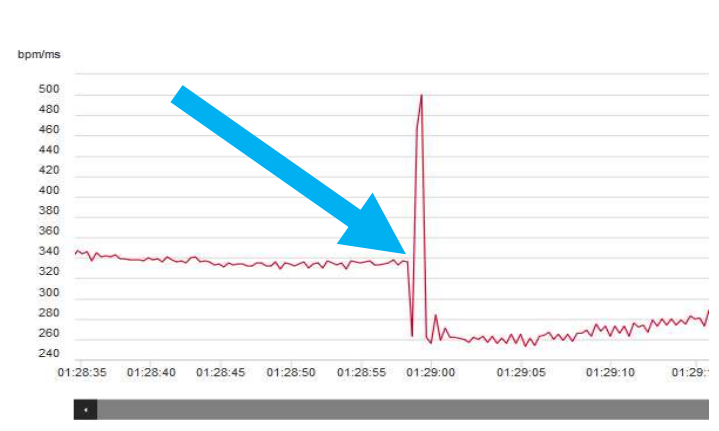

Figure D-1: Indicating R-R Start Time

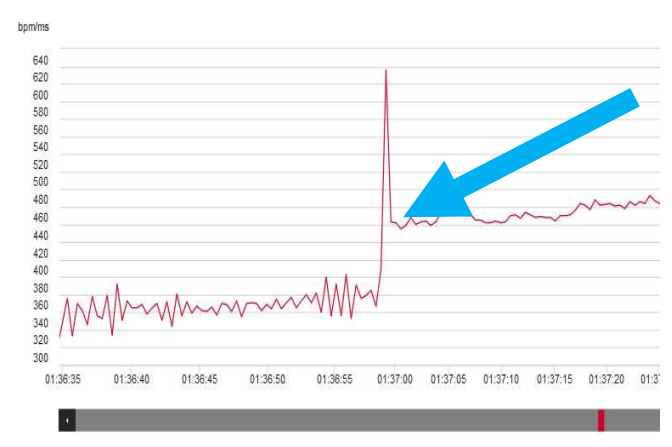

Figure D-2: Indicating R-R End Time

$\underline{\text { R-R Duration (h:mm:ss): }}$ This column provides for total time that the occurrence took place.

For calculating this variable, subtract R-R Start Time from R-R End Time 
Peak HR (bpm): This is the highest HR reached during the identified occurrence. This was determined by visually identifying the highest point reached and then placing the cursor on that point to identify what the numeric value is displayed.

Peak R-R (bpm): This is the highest HR reached during the identified occurrence as determined by $\mathrm{R}-\mathrm{R}$ value and not the HR tracing. This value was determined by dividing 60 by the value from "peak" bpm/ms.

Peak HR vs Peak RR Difference (bpm): This value is the difference in HR as calculated between the peak HR from the HR tracing and what was calculated from the R-R data. This value is determined by subtracting the value in "Peak HR (bpm) from "Peak R-R (bpm). Init Artifact: This column provides indication as to if the initial HR acceleration data for this occurrence meets criteria to be classified as artifact, and possibly disqualifying the occurrence as non-arrhythmic in nature. To verify, RRI start and stop times should also be present if an actual occurrence has occurred or it is truly artifact in nature. This column is based on the values located in the Init Slope column. In this case the slope must be less than 0.1 to be considered artifact.

Term Artifact: This column provides indication as to if the termination HR deceleration data for this occurrence meets criteria to be classified as artifact, and possibly disqualifying the occurrence as non-arrhythmic in nature. To verify, RRI start and stop times should also be present if an actual occurrence has occurred or it is truly artifact in nature. This column is based on the values located in the Term Slope column. In this case the slope must be less than 0.1 to be considered artifact. 
Outside of Recorded Session: This column allows for quicker analysis of occurrences that may have taken place outside of a recorded HR session. The sensors used by this system continually record data in RR Interval when worn by the athlete. The term recorded session in this case refers to when the coaching staff wishes to record a specific activity, practice, or match which will provide more variables for future analysis. Occurrences recorded outside of a recorded session are identified by analysis of the RR Interval function, and are annotated in the document via analyst input.

Indeterminate HR Tracing: This column is used to identify multiple occurrences that take place in such close proximity on the HR tracing that actual definitive values to determine initiation and termination cannot be determined. What may appear as one occurrence on the HR tracing may in fact be multiple occurrences. In this instance initiation and termination times can be determined using RRI.

Artifact Occurrence: This column allows to identify if an occurrence can be discounted due to classification as artifact. This is determined by if the "Init Artifact" and "Term Artifact" columns meet criteria as artifact.

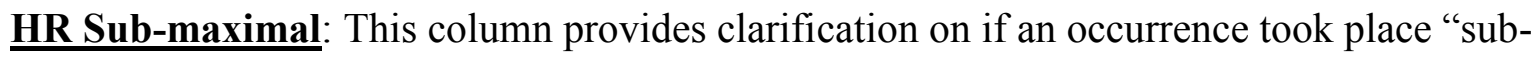
maximally", or did not peak above the subject's maximum HR value. This is determined by calculating, via formula if the value in "Peak HR (bpm)" was greater than $190 \mathrm{bpm}$.

R-R Sub-maximal: This column provides clarification of if an occurrence took place "submaximally", or did not peak above the subject's maximum HR value. This is determined by calculating, via formula, if the value in "Peak R-R (bpm)" was greater than $190 \mathrm{bpm}$. 